\title{
A systematic review of the relationship between subchondral bone features, pain and structural pathology in peripheral joint osteoarthritis
}

\author{
Andrew J. Barr' ${ }^{1}$, T. Mark Campbell ${ }^{1,2}$, Devan Hopkinson³, Sarah R. Kingsbury', Mike A. Bowes ${ }^{3}$ \\ and Philip G. Conaghan ${ }^{1 *}$
}

\begin{abstract}
Introduction: Bone is an integral part of the osteoarthritis $(\mathrm{OA})$ process. We conducted a systematic literature review in order to understand the relationship between non-conventional radiographic imaging of subchondral bone, pain, structural pathology and joint replacement in peripheral joint OA.

Methods: A search of the Medline, EMBASE and Cochrane library databases was performed for original articles reporting association between non-conventional radiographic imaging-assessed subchondral bone pathologies and joint replacement, pain or structural progression in knee, hip, hand, ankle and foot OA. Each association was qualitatively characterised by a synthesis of the data from each analysis based upon study design, adequacy of covariate adjustment and quality scoring.

Results: In total 2456 abstracts were screened and 139 papers were included (70 cross-sectional, 71 longitudinal analyses; 116 knee, 15 hip, six hand, two ankle and involved 113 MRl, eight DXA, four CT, eight scintigraphic and eight 2D shape analyses). BMLs, osteophytes and bone shape were independently associated with structural progression or joint replacement. BMLs and bone shape were independently associated with longitudinal change in pain and incident frequent knee pain respectively.
\end{abstract}

Conclusion: Subchondral bone features have independent associations with structural progression, pain and joint replacement in peripheral OA in the hip and hand but especially in the knee. For peripheral OA sites other than the knee, there are fewer associations and independent associations of bone pathologies with these important OA outcomes which may reflect fewer studies; for example the foot and ankle were poorly studied. Subchondral OA bone appears to be a relevant therapeutic target.

Systematic review: PROSPERO registration number: CRD 42013005009

\section{Introduction}

Osteoarthritis (OA), the most common form of arthritis, is a major cause of chronic pain and disability. OA confers a huge burden on both individuals and health economies $[1,2]$. There are currently no licensed disease-modifying osteoarthritis drugs (DMOADs) but ideally these should

\footnotetext{
* Correspondence: p.conaghan@leeds.ac.uk

'Leeds Institute of Rheumatic and Musculoskeletal Medicine, University of Leeds and NIHR Leeds Musculoskeletal Biomedical Research Unit, Chapeltown Rd, Leeds LS7 4SA, UK

Full list of author information is available at the end of the article
}

both inhibit structural progression and improve symptoms and/or function [3, 4]. While hyaline cartilage loss is the hallmark pathology, clinical OA usually involves multiple tissues. Describing the relationships of these tissues with structural progression and symptoms may identify potential tissue targets.

The subchondral bone in particular is intimately associated with hyaline cartilage and therefore a tissue of great potential interest. Conventional radiographs are known to be relatively insensitive to the structural features of $\mathrm{OA}$ [5], in part because they do not assess three-dimensional

\section{Ciomed Central}

(c) 2015 Barr et al. Open Access This article is distributed under the terms of the Creative Commons Attribution 4.0 International License (http://creativecommons.org/licenses/by/4.0/), which permits unrestricted use, distribution, and reproduction in any medium, provided you give appropriate credit to the original author(s) and the source, provide a link to the Creative Commons license, and indicate if changes were made. The Creative Commons Public Domain Dedication waiver (http://creativecommons.org/publicdomain/zero/1.0/) applies to the data made available in this article, unless otherwise stated. 
(3D) bone structure [6]. A number of non-conventional radiographic imaging modalities accurately demonstrate in vivo subchondral bone pathological changes, including magnetic resonance imaging (MRI), computed tomography (CT), dual-energy x-ray absorptiometry (DXA), scintigraphy and positron emission tomography (PET) [5, 7-13]. Hunter and colleagues found a moderate association between bone marrow lesions (BMLs), structural progression and longitudinal change in pain in a systematic review focused on MRI biomarkers and knee OA [7]. In another systematic review Kloppenburg and colleagues examined associations between MRI features and knee pain, but not structural pathology [14].

We therefore wished to comprehensively review the literature on subchondral bone structure assessed with all non-conventional radiographic imaging modalities, examining the common sites of peripheral OA and describing the relationships between imaging-detected subchondral bone features and joint replacement, structural progression and pain.

\section{Methods}

Systematic literature search

A systematic literature search of Medline (from 1950), EMBASE (from 1980) and the Cochrane library databases until September 2014 was performed. A full description of the search terms used is recorded in Additional file 1: Table S1. An abbreviation of the full search terms used was 'knee, hip, hand, foot and ankle' and 'osteoarthritis' and 'subchondral bone' manifestations of OA ('bone marrow lesion,' 'osteophyte, 'bone cyst,' 'bone area, 'bone shape, 'bone attrition', bone morphometry and mineral density) and 'MRI' or 'CT' or 'DXA' or 'scintigraphy' or 'PET'. The search term 'bone shape' was not restricted to non-conventional radiographic imaging. The final search was restricted to humans. There was no language restriction and abstracts were not excluded. Exclusion criteria are listed in Fig. 1. Any analysis of fewer than 20 patients with confirmed OA was excluded to remove papers at risk of study imprecision. The inclusion criteria were in vivo observational studies of a human population with clinical

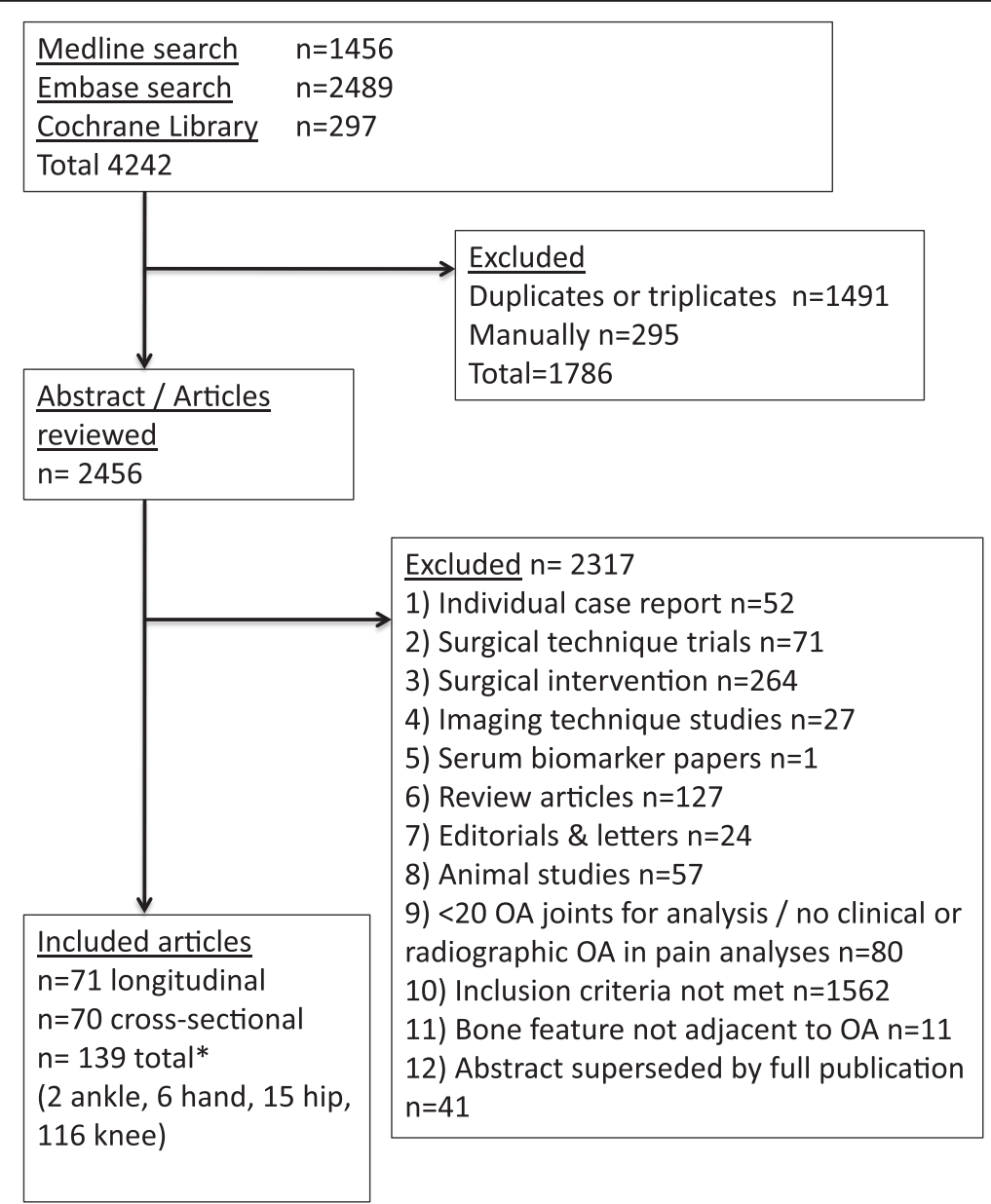

Fig. 1 Search strategy results and article exclusion. ${ }^{*}$ Two articles included both cross-sectional and longitudinal data. Longitudinal data included 16 case-control studies and 55 cohort studies 
and/or radiographic OA, which included an imaging description of the adjacent subchondral bone pathology to the osteoarthritic joint and the relationship of this with pain, structural progression or joint replacement. Analyses describing the relationship between OA bone manifestations and structural severity (cross-sectional) or progression (prospective cohorts) in populations without clinical and radiographic OA were included to incorporate early structural features of joint degeneration. The outcome measures of structural severity or progression included cartilage defects, cartilage thickness, cartilage volume, denuded subchondral bone, Kellgren-Lawrence (KL)grade, joint space width and joint space narrowing. Other outcome measures included joint replacement and any pain measures.

The articles identified by the preliminary search were screened by two reviewers (DH, AB) for relevance and for references not identified by the preliminary search, although no additional citations were found. Discordance in opinion was resolved by a third reviewer (SK). We applied the methods for reporting meta-analyses of observational studies in epidemiology that are recommended by the Cochrane collaboration $[15,16]$.

\section{Data extraction}

Data extraction was performed by two reviewers $(\mathrm{DH}, \mathrm{AB})$ as described in the Supplementary methods 'data extraction' (see Additional file 1).

\section{Quality assessment}

The quality of each observational study was independently assessed by two reviewers (TC, AB), as described in Supplementary methods 'Quality assessment' (see Additional file 1).

\section{Best evidence synthesis}

Statistical pooling of the data was considered inappropriate in light of the heterogeneous study populations, methodological quality and bone feature or outcome measurements for OA. Therefore a qualitative summary of the evidence for each bone feature (e.g., BML) and its association with pain or structural progression and joint replacement was provided based on the study design, adequacy of adjustment for confounders (age, body mass index and gender) and quality score as described in the Supplementary methods 'Best evidence synthesis' (see Additional file 1).

Studies that investigated the association between multiple bone features and OA pain or structural progression outcomes were considered as a single study for each bone feature. Included studies that established significant correlation between bone and pain, structural progression or joint replacement were described as positive $(+)$ or negative (-) accordingly. If no association or inconclusive findings were described this was reported as no association (NA) or no conclusion (NC) respectively.

\section{Results}

Systematic literature search and selection

The Preferred reporting items for systematic reviews and meta-analyses (PRISMA) diagram in Fig. 1 describes the literature flow. Following exclusion of duplicates and triplicates, 2,456 articles met the search criteria. After applying inclusion/exclusion criteria, 139 articles were included for data extraction and quality scoring. In total, 71 papers provided longitudinal data (55 cohorts, 16 case-controls), 70 provided cross-sectional data, and two papers provided both.

\section{Data extraction from selected studies}

In only 12 studies did the mean age fall below 50 years [17-29]. Most $(n=93)$ described both genders; 2 studies included men only [27, 30], 14 studies included female individuals only $[22,28,29,31-41]$ and there was an undisclosed gender ratio in 6 [42-50]. Knee OA was defined using clinical and radiographic criteria and is described in Additional file 1: Table S6. Radiographic OA was invariably defined as KL grade $\geq 2$ or any radiographic OA abnormality from the Altman atlas [51]. Individual pain or structural progression measures were examined in 88 studies; 52 studies examined multiple features. Subchondral bone was analyzed with MRI in 113 articles, DXA in 8 [30-32, 42, 52-55], CT in 4 [33, 40, 56, 57], and scintigraphy in $8[24,37,38,58-62]$, and no articles using PET met the inclusion criteria. Included articles described 116 knee, 15 hip, 6 hand and 2 ankle studies. Of these studies 13 described structural associations without clinical or radiographic OA $[18,19,23,25,26$, 35, 63-69]. There were no articles on studies of the foot that met the inclusion criteria.

\section{Quality assessment of studies}

Concordance of opinion in quality scoring was observed in 2,040 (89\%) of the 2,242 scoring items assessed, which are recorded in Additional file 1: Tables S3-S5. The majority of discordant scoring was for study design (criteria 17) and data presentation (criteria 18). Quality scores were converted to percentages of the maximum scores for each class of paper. The mean (range) quality score was $59 \%(29-79), 54 \%(22-83)$ and $59 \%(47-76)$ for crosssectional, cohort and case-control studies, respectively.

\section{Relationship between knee bone feature and structural progression}

The association of bone features with structural progression and joint replacement are described in Tables 1 and 5. 
Table 1 Knee structural associations by feature and quality grade

\begin{tabular}{|c|c|c|c|c|c|c|c|}
\hline Author & Feature (method) & $\begin{array}{l}\text { Structural progression } \\
\text { outcome }\end{array}$ & $\begin{array}{l}\text { Adjustment for } \\
\text { confounders }\end{array}$ & $\begin{array}{l}\text { Association (magnitude) } \\
\text { crude }\end{array}$ & $\begin{array}{l}\text { Association (magnitude) } \\
\text { adjusted }\end{array}$ & Association & $\begin{array}{l}\text { Quality } \\
\text { (score \%) }\end{array}$ \\
\hline \multicolumn{8}{|c|}{ MRI bone marrow lesion - cohorts } \\
\hline \multirow[t]{2}{*}{ Felson 2003 [70] } & \multirow{2}{*}{$\begin{array}{l}\text { Baseline presence of BML } \\
\text { in medial or lateral TFJ (C) }\end{array}$} & \multirow{2}{*}{$\begin{array}{l}\text { OARSI JSN grade } \\
\text { progression of } \\
\text { TFJ (L) }\end{array}$} & \multirow[t]{2}{*}{ Age, sex, and BMl } & \multirow[t]{2}{*}{ NR } & OR 6.5, & \multirow[t]{2}{*}{+} & \multirow[t]{2}{*}{ High (83) } \\
\hline & & & & & $95 \%$ Cl 3.0 to 14.0 & & \\
\hline \multirow[t]{3}{*}{ Dore 2010 [124] } & \multirow{3}{*}{$\begin{array}{l}\text { Baseline semi-quantitative } \\
\text { MRI BML size (C) TFJ }\end{array}$} & \multirow{3}{*}{$\begin{array}{l}\text { Incident TKR over } \\
5 \text { years }(\mathrm{L})\end{array}$} & \multirow{3}{*}{$\begin{array}{l}\text { Age, sex, BMl, knee } \\
\text { baseline pain, leg } \\
\text { strength, cartilage } \\
\text { defects, tibial bone } \\
\text { area, ROA }\end{array}$} & OR $(95 \% \mathrm{Cl})$ & OR $(95 \% \mathrm{Cl})$ & \multirow[t]{3}{*}{+} & \multirow[t]{3}{*}{ High (64) } \\
\hline & & & & 2.04 (1.55 to 2.69$)$ & $2.10(1.13$ to 3.90$)$ & & \\
\hline & & & & $p<0.01$ & $p=0.019$ & & \\
\hline \multirow[t]{4}{*}{ Driban 2013 [72] } & \multirow{2}{*}{$\begin{array}{l}\text { Knee baseline BML } \\
\text { volume }(C)\end{array}$} & \multirow{2}{*}{$\begin{array}{l}\text { 48-month change in } \\
\text { OARSI JSN grade }(L)\end{array}$} & \multirow[t]{4}{*}{ Age, sex, BMl } & \multirow[t]{4}{*}{ NR } & Baseline BML volume & \multirow[t]{4}{*}{+} & \multirow[t]{4}{*}{ High (61) } \\
\hline & & & & & $\begin{array}{l}\text { OR } 1.27,95 \% \mathrm{Cl} 1.11 \\
\text { to } 1.46\end{array}$ & & \\
\hline & \multirow{2}{*}{$\begin{array}{l}\text { BML volume } 48 \text { month } \\
\text { change (L) (TFJ) }\end{array}$} & \multirow[t]{2}{*}{$(\mathrm{TFJ})$} & & & BML volume regression & & \\
\hline & & & & & $\begin{array}{l}\text { OR } 3.36,95 \% \mathrm{Cl} 1.55 \\
\text { to } 7.28\end{array}$ & & \\
\hline \multirow{5}{*}{$\begin{array}{l}\text { Davies-Tuck } \\
2010[67]\end{array}$} & \multirow{5}{*}{$\begin{array}{l}\text { Incident BML (new BML } \\
\text { after } 2 \text { years with no BMLs } \\
\text { at baseline) MRI TFJ (L) }\end{array}$} & \multirow{5}{*}{$\begin{array}{l}\text { Progression in semi- } \\
\text { quantitative MRI } \\
\text { cartilage defects score } \\
\text { after } 2 \text { years. TFJ }(L)\end{array}$} & \multirow{5}{*}{$\begin{array}{l}\text { Age, gender, BMI, } \\
\text { baseline cartilage } \\
\text { volume }\end{array}$} & OR $(95 \%$ Cl) & OR $(95 \% \mathrm{Cl})$ & + & High (61) \\
\hline & & & & Medial TFJ & Medial TFJ & \multirow{4}{*}{$\begin{array}{l}\text { Association in the } \\
\text { lateral TFJ and a } \\
\text { trend in the medial } \\
\text { TFJ }\end{array}$} & \\
\hline & & & & 1.86 (0.70 to 4.93$) p=0.21$ & $\begin{array}{l}2.63(0.93 \text { to } 7.44) \\
p=0.07\end{array}$ & & \\
\hline & & & & Lateral TFJ & Lateral TFJ & & \\
\hline & & & & $3.0(1.01$ to 8.93$) p=0.05$ & $\begin{array}{l}3.13(1.01 \text { to } 9.68) \\
p=0.05\end{array}$ & & \\
\hline \multirow[t]{2}{*}{ Hochberg 2014 [44] } & \multirow{2}{*}{$\begin{array}{l}\text { Semi-quantitative MRI } \\
\text { baseline femoral condyle } \\
\text { BML size }(C)\end{array}$} & \multirow{2}{*}{$\begin{array}{l}\text { Incident TKR over } \\
6 \text { years }(L)\end{array}$} & \multirow{2}{*}{$\begin{array}{l}\text { Age, gender, BMI, } \\
\text { race, marital status, } \\
\text { depressive symptoms, } \\
\text { quality of life, mechanical } \\
\text { pain, KL grade, clinical } \\
\text { effusion. }\end{array}$} & Medial TFJ & Medial TFJ & \multirow[t]{2}{*}{+} & High (61) \\
\hline & & & & $p<0.0001$ & $p=0.02$ & & \\
\hline Raynauld 2011 [75] & Baseline semi-quantitative & Incidence of TKR over & Age, sex, BMI, JSW, & NR & OR $(95 \% \mathrm{Cl})$ & + & High (61) \\
\hline & & & & & BML medial plateau & & \\
\hline & & & & & 1.81 (1.08 to 2.03$)$ & & \\
\hline & & & & & $p=0.025$ & & \\
\hline Raynauld 2013 [74] & Baseline semi-quantitative & Incident TKR (L) & Age, BMI, gender & NR & TKR incidence & + & High (61) \\
\hline & $\begin{array}{l}\text { BML WORMS score (C) } \\
\text { medial TEJ }\end{array}$ & 4 year follow up & WOMAC, CRP & & OR (95\% Cl) 2.107 & & \\
\hline & & Time to TKR $(\mathrm{L})$ & & & $\begin{array}{l}(1.26 \text { to } 3.54) p=0.005 \\
\text { time to TKR incidence } \\
\text { hazard ratio }(95 \% \text { Cl) } \\
2.13(1.38 \text { to } 3.30) \\
p=0.001\end{array}$ & & \\
\hline
\end{tabular}


Table 1 Knee structural associations by feature and quality grade (Continued)

\begin{tabular}{|c|c|c|c|c|c|c|c|}
\hline \multirow[t]{2}{*}{ Crema 2014 [71] } & MRI BML (semi-quantitative) & $\begin{array}{l}\text { Cartilage loss (semi- } \\
\text { quantitative) }\end{array}$ & Age, gender, BMl & NR & $\begin{array}{l}\beta=0.37 \text { to } 0.64 \\
p<0.001\end{array}$ & + & High (56) \\
\hline & (C) all regions & (L) (all regions) & & & & & \\
\hline \multirow[t]{2}{*}{$\begin{array}{l}\text { Guermazi } 2014 \\
\text { Abstract [73] }\end{array}$} & \multirow[t]{2}{*}{$\begin{array}{l}\text { Baseline semi-quantitative } \\
\text { BML score WORMS (C) }\end{array}$} & \multirow[t]{2}{*}{$\begin{array}{l}\text { Cartilage thickness loss } \\
\text { over } 30 \text { months }(\mathrm{L})\end{array}$} & \multirow[t]{2}{*}{$\begin{array}{l}\text { Age, sex, body mass } \\
\text { index, and anatomical } \\
\text { alignment axis (degrees) }\end{array}$} & \multirow[t]{2}{*}{ NR } & $\begin{array}{l}\text { Combined BML score } \\
\text { in the medial and } \\
\text { lateral TFJ compartment }\end{array}$ & \multirow[t]{2}{*}{+} & \multirow[t]{2}{*}{ High (56) } \\
\hline & & & & & $\begin{array}{l}\text { OR } 1.9,95 \% \mathrm{Cl} 1.1 \\
\text { to } 3.3\end{array}$ & & \\
\hline \multirow[t]{3}{*}{ Scher 2008 [87] } & \multirow{3}{*}{$\begin{array}{l}\text { Presence of any baseline } \\
\text { semi-quantitative MRI } \\
\text { BMLs (C) }\end{array}$} & \multirow{3}{*}{$\begin{array}{l}\text { Incident TKR }(L) \text { over } \\
3 \text { years }\end{array}$} & \multirow[t]{3}{*}{ Age } & \multirow[t]{3}{*}{ NR } & OR $(95 \%$ Cl) & \multirow[t]{3}{*}{+} & \multirow[t]{3}{*}{ High (56) } \\
\hline & & & & & 8.95 (1.49 to 53.68$)$ & & \\
\hline & & & & & $p=0.02$ & & \\
\hline \multirow[t]{2}{*}{ Sowers 2011 [28] } & \multirow[t]{2}{*}{$\begin{array}{l}\text { Semi-quantitative MRI } \\
\text { BML, size in TFJ (C) }\end{array}$} & $\begin{array}{l}\text { Progression in } \mathrm{KL} \\
\text { grade }\end{array}$ & \multirow[t]{2}{*}{ Nil } & $\begin{array}{l}R(95 \% \mathrm{Cl}) \text { medial tibia } \\
0.46(0.35 \text { to } 0.55)\end{array}$ & NR & \multirow[t]{2}{*}{+} & \multirow[t]{2}{*}{ Low (53) } \\
\hline & & (11-year follow up) (L) & & $\begin{array}{l}\text { Lateral tibia } \sim 0.23(0.13 \\
\text { to } 0.33)\end{array}$ & & & \\
\hline \multirow[t]{2}{*}{ Kothari 2010 [82] } & \multirow{2}{*}{$\begin{array}{l}\text { Semi-quantitative baseline } \\
\text { MRI BML, (WORMS) (C) TFJ }\end{array}$} & \multirow{2}{*}{$\begin{array}{l}\text { Semi-quantitative } \\
\text { cartilage defect score } \\
\text { change over } 2 \text { years } \\
\text { (WORMS) (L) TFJ. }\end{array}$} & \multirow{2}{*}{$\begin{array}{l}\text { Age, sex, BMl, other bone } \\
\text { lesions }\end{array}$} & OR 4.04, & OR 3.75, & \multirow[t]{2}{*}{+} & \multirow[t]{2}{*}{ Low (50) } \\
\hline & & & & $95 \%$ Cl 2.25 to 7.26 & $95 \% \mathrm{Cl} 1.59$ to 8.82 & & \\
\hline \multirow[t]{5}{*}{ Raynauld 2008 [85] } & \multirow{5}{*}{$\begin{array}{l}\text { Change in BML size (mm) } \\
\text { at } 24 \text { months in medial TFJ (L) }\end{array}$} & \multirow{5}{*}{$\begin{array}{l}\text { Medial cartilage } \\
\text { volume }(L) \text { at } 24 \\
\text { months in medial TFJ }\end{array}$} & \multirow{5}{*}{$\begin{array}{l}\text { Age, gender, BMl, } \\
\text { meniscal extrusion } \\
\text { and tear, pain and } \\
\text { bone lesions at baseline }\end{array}$} & \multirow[t]{5}{*}{ NR } & Change in BML size & \multirow{5}{*}{$\begin{array}{l}\text { - } \\
\text { Larger medial BML } \\
\text { size means more } \\
\text { cartilage loss in } \\
\text { medial compartment }\end{array}$} & \multirow[t]{5}{*}{ Low (50) } \\
\hline & & & & & $\begin{array}{l}\text { With femoral cartilage } \\
\text { volume loss }\end{array}$ & & \\
\hline & & & & & $\beta=-0.31$ & & \\
\hline & & & & & standard error (0.08) & & \\
\hline & & & & & $p=0.0004$ & & \\
\hline Roemer 2009 [90] & Change in MRI semi- & Progression in semi- & Age, sex, BMI, baseline & NR & OR $(95 \% \mathrm{Cl})$ & + & Low (50) \\
\hline & $\begin{array}{l}\text { quantitative BML size } \\
\text { (WORMS) (L) TFJ and PFJ }\end{array}$ & $\begin{array}{l}\text { quaneitative cartilage } \\
\text { defects in (WORMS) } \\
\text { over } 30 \text { months (L) TFJ }\end{array}$ & KL grade & & $\begin{array}{l}\text { Incident BML OR } 3.5 \\
\text { (2.1 to } 5.9)\end{array}$ & & \\
\hline & & and PFJ & & & $\begin{array}{l}\text { Progression of BML } \\
2.8 \text { (1.5 to } 3.2)\end{array}$ & & \\
\hline & & & & & $\begin{array}{l}\text { Resolution of BML OR } \\
0.9(0.5 \text { to } 1.6)\end{array}$ & & \\
\hline & & & & & $\begin{array}{l}\text { Stable BML OR } 1.0 \\
\text { (reference) }\end{array}$ & & \\
\hline Dore 2010 [76] & Baseline semi-quantitative & Ipsi-compartmental & Age, sex, BMI, meniscal & NR & Baseline & - & Low (50) \\
\hline & and lateral TFJ) & volume loss (L) & admage & & BML severity & Bigger BML means & \\
\hline & & & & & $\begin{array}{l}\beta=-22.1 \text { to }-42.0, \\
\text { for all regions }\end{array}$ & me loss & \\
\hline & & & & & $(p<0.05)$ & & \\
\hline
\end{tabular}


Table 1 Knee structural associations by feature and quality grade (Continued)

\begin{tabular}{|c|c|c|c|c|c|c|c|}
\hline $\begin{array}{l}\text { Parsons } 2014 \\
\text { Abstract [83] }\end{array}$ & $\begin{array}{l}\text { Baseline semi-quantitative } \\
\text { BML score (C) }\end{array}$ & Annual TFJ JSN (L) & $\begin{array}{l}\text { Age, sex, baseline } \mathrm{KL} \\
\text { grade }\end{array}$ & NR & $\begin{array}{l}\beta=-0.10,95 \% \mathrm{Cl} \\
-0.18 \text { to } \\
-0.02\end{array}$ & + & Low (50) \\
\hline Wildi 2010 [95] & $\begin{array}{l}\text { 24-month regional } \\
\text { change in TFJ BML } \\
\text { score WORMS }(L)\end{array}$ & $\begin{array}{l}\text { 24-month regional } \\
\text { change in cartilage } \\
\text { volume }(\mathrm{L})\end{array}$ & nil & $\begin{array}{l}R \text { correlation coefficients } \\
\text { all }<0.07 \\
p>0.367 \text { for all three } \\
\text { compartments at } 24 \\
\text { months }\end{array}$ & $N R$ & NC & Low (50) \\
\hline Pelletier 2007 [84] & $\begin{array}{l}\text { Regional Semi-quantitative } \\
\text { baseline BML score (medial } \\
\text { or lateral TFJ) (C) }\end{array}$ & $\begin{array}{l}\text { Regional cartilage } \\
\text { volume over } 24 \\
\text { months (medial } \\
\text { or lateral TFJ) (L) }\end{array}$ & $N R$ & $\begin{array}{l}\text { Lateral compartment BML } \\
\text { score } \\
\beta=-0.31, p=0.001\end{array}$ & $N R$ & & Low (50) \\
\hline Driban 2011 [79] & $\begin{array}{l}\text { Baseline BML volume }(C) \\
\text { and } 24 \text { month change in } \\
\text { BML volume }(\mathrm{L}) \text { in TFJ } \\
\text { compartments }\end{array}$ & $\begin{array}{l}\text { 24-month change in } \\
\text { full thickness cartilage } \\
\text { lesion area }(\mathrm{L})\end{array}$ & $\begin{array}{l}\text { Age, sex, body mass } \\
\text { index }\end{array}$ & NR & $\begin{array}{l}\text { Baseline BML volume } \\
r=0.48,95 \% \mathrm{Cl} 0.20 \\
\text { to } 0.69 \\
p<0.002\end{array}$ & $\begin{array}{l}+ \\
\text { Baseline femur BML } \\
\text { volume with loss in } \\
\text { ipsicompartmental } \\
\text { full thickness } \\
\text { cartilage lesion area. }\end{array}$ & Low (50) \\
\hline Tanamas 2010 [89] & $\begin{array}{l}\text { Baseline semi-quantitative } \\
\text { MRI BML size (C) TFJ }\end{array}$ & $\begin{array}{l}\text { Cartilage volume } \\
\text { change over } 2 \text { years } \\
\text { (L) TFJ Incident TKR } \\
\text { over } 4 \text { years }\end{array}$ & $\begin{array}{l}\text { Age, sex, BMl, baseline } \\
\text { tibial cartilage volume } \\
\text { and bone area }\end{array}$ & $\begin{array}{l}R(95 \% \mathrm{Cl}) \\
\text { Total cartilage loss } \\
0.61(-0.11 \text { to } 1.33) \\
\text { OR }(95 \% \mathrm{Cl}) \\
\text { Incident TKR } \\
1.55(1.04 \text { to } 2.29) \\
p=0.03\end{array}$ & $\begin{array}{l}R(95 \% \mathrm{Cl}) \\
\text { Total cartilage loss } \\
1.09(0.24,1.93) \\
\text { OR }(95 \% \mathrm{Cl}) \\
\text { Incident TKR } \\
1.57(1.04 \text { to } 2.35) \\
p=0.03\end{array}$ & + & Low (50) \\
\hline $\begin{array}{l}\text { Madan-Sharma } \\
2008 \text { [93] }\end{array}$ & $\begin{array}{l}\text { Baseline MRI semi- } \\
\text { quantitative BML (C) TFJ }\end{array}$ & $\begin{array}{l}\text { OARSI medial TFJ JSN } \\
\text { grade progression } \\
\text { over } 2 \text { years (L) TFJ }\end{array}$ & $\begin{array}{l}\text { Age, sex, BMI, family } \\
\text { effect }\end{array}$ & NR & $\begin{array}{l}0.9 \text { RR, } \\
95 \% \text { Cl } 0.18 \text { to } 3.0\end{array}$ & NA & Low (47) \\
\hline Tanamas 2010 [88] & $\begin{array}{l}\text { Semi-quantitative change } \\
\text { in MRI BML severity (C) }\end{array}$ & $\begin{array}{l}\text { Incident TKR over } \\
4 \text { years }(L)\end{array}$ & Age, gender, $\mathrm{KL}$ grade & $\begin{array}{l}\text { OR }(95 \% \mathrm{Cl}) \\
\text { Medial TFJ } \\
1.72 \\
(0.93 \text { to } 3.18) \\
p=0.08 \\
\text { Lateral TFJ } \\
0.95 \text { (0.48 to 1.88) } \\
p=0.89\end{array}$ & $\begin{array}{l}\text { OR }(95 \% \text { Cl }) \\
\text { Medial TFJ } \\
1.99 \\
(1.01 \text { to } 3.90) \\
p=0.05 \\
\text { Lateral TFJ } \\
0.96 \text { (0.48 to } 1.94) \\
p=0.91\end{array}$ & $\begin{array}{l}+ \\
\text { Association in the } \\
\text { medial TFJ but not } \\
\text { in the lateral TFJ }\end{array}$ & Low (47) \\
\hline Roemer 2012 [86] & $\begin{array}{l}\text { Semi-quantitative BML } \\
\text { (WORMS) TFJ and PFJ (C) }\end{array}$ & $\begin{array}{l}\text { Semi-quantitative } \\
\text { cartilage score }\end{array}$ & $\begin{array}{l}\text { Age, sex, treatment, and } \\
\text { BMI. }\end{array}$ & $N R$ & $\begin{array}{l}\text { BML TFJ OR } 4.74,95 \% \\
\text { Cl } 1.14 \text { to } 19.5\end{array}$ & + & Low (44) \\
\hline
\end{tabular}


Table 1 Knee structural associations by feature and quality grade (Continued)

\begin{tabular}{|c|c|c|c|c|c|c|c|}
\hline & & \multirow{2}{*}{$\begin{array}{l}\text { 6-month progression } \\
\text { TFJ and PFJ (L) }\end{array}$} & & & $p=0.032$ & \multirow{2}{*}{$\begin{array}{l}\text { BMLs and cartilage } \\
\text { score correlate }\end{array}$} & \\
\hline & & & & & $\begin{array}{l}\text { BML PFJ OR, } 1.63(0.67 \\
\text { to } 3.92)\end{array}$ & & \\
\hline \multirow[t]{5}{*}{ Crema 2013 [78] } & MRI incident BML (WORMS) & \multirow{5}{*}{$\begin{array}{l}\text { Progressive (30 } \\
\text { month) semi- } \\
\text { quantitative } \\
\text { cartilage defect } \\
\text { (WORMS) TFJ (L) }\end{array}$} & \multirow{5}{*}{$\begin{array}{l}\text { Age, sex, BMI, malalignment, } \\
\text { meniscal disease }\end{array}$} & \multirow[t]{5}{*}{$N R$} & OR $(95 \% \mathrm{Cl})$ & \multirow[t]{5}{*}{+} & \multirow[t]{5}{*}{ Low (44) } \\
\hline & TFJ & & & & Medial TFJ 7.6 & & \\
\hline & \multirow[t]{3}{*}{$(\mathrm{L})$} & & & & (5.1 to 11.3 ) & & \\
\hline & & & & & Lateral TFJ & & \\
\hline & & & & & 11.9 (6.2 to 23.0$)$ & & \\
\hline \multirow[t]{3}{*}{$\begin{array}{l}\text { Hernandez-Molina } \\
2008 \text { [81] }\end{array}$} & \multirow[t]{3}{*}{$\begin{array}{l}\text { Crude presence of central } \\
\text { BMLs on MRI (C) TFJ }\end{array}$} & \multirow{3}{*}{$\begin{array}{l}\text { Semi-quantitative } \\
\text { cartilage defect } \\
\text { (WORMS) (L) TFJ }\end{array}$} & \multirow[t]{3}{*}{$\begin{array}{l}\text { Alignment, BMI, KL grade, } \\
\text { sex, and age. }\end{array}$} & \multirow[t]{3}{*}{ NR } & $\begin{array}{l}\text { Medial TFJ cartilage } \\
\text { loss }\end{array}$ & \multirow[t]{3}{*}{+} & \multirow[t]{3}{*}{ Low (44) } \\
\hline & & & & & OR 6.1, & & \\
\hline & & & & & $95 \%$ Cl 1.0, 35.2 & & \\
\hline \multirow[t]{3}{*}{ Koster 2011 [25] } & \multirow{3}{*}{$\begin{array}{l}\text { Baseline BML presence } \\
\text { (C) TFJ }\end{array}$} & \multirow{3}{*}{$\begin{array}{l}\text { Any progression in } \\
\text { KL grade over } 1 \text { year } \\
\text { (L) TFJ }\end{array}$} & \multirow[t]{3}{*}{ Age, BMl } & OR $(95 \%$ Cl) & OR $(95 \% \mathrm{Cl})$ & \multirow[t]{3}{*}{+} & \multirow[t]{3}{*}{ Low (44) } \\
\hline & & & & 6.01 (1.92 to 18.8$)$ & $5.29(1.64$ to 17.1$)$ & & \\
\hline & & & & $p=0.002$ & $p=0.005$ & & \\
\hline \multirow[t]{6}{*}{ Hunter 2006 [91] } & \multirow{6}{*}{$\begin{array}{l}\text { Change in MRI semi- } \\
\text { quantitative BML score } \\
\text { (L) TFJ }\end{array}$} & \multirow{6}{*}{$\begin{array}{l}\text { Change in semi- } \\
\text { quantitative cartilage } \\
\text { defect score (WORMS) } \\
\text { (L) medial or lateral } \\
\text { TFJ }\end{array}$} & \multirow[t]{6}{*}{ Limb alignment } & Ipsilateral cartilage loss & $\begin{array}{l}\text { Ipsilateral cartilage } \\
\text { loss }\end{array}$ & \multirow[t]{6}{*}{ NA after adjustment } & \multirow[t]{6}{*}{ Low (44) } \\
\hline & & & & $\beta=0.65$ & $\beta=0.26$ & & \\
\hline & & & & $p=0.003$ & $p=0.16$ & & \\
\hline & & & & $\begin{array}{l}\text { Contralateral cartilage } \\
\text { loss }\end{array}$ & $\begin{array}{l}\text { Contralateral cartilage } \\
\text { loss }\end{array}$ & & \\
\hline & & & & $\beta=-0.27$ & $\beta=-0.16$ & & \\
\hline & & & & $p=0.22$ & $p=0.52$ & & \\
\hline \multirow[t]{3}{*}{ Roemer 2009 [94] } & \multirow{3}{*}{$\begin{array}{l}\text { Baseline MRI BML crude } \\
\text { presence or absence } \\
\text { (WORMS) (L) TFJ }\end{array}$} & \multirow{3}{*}{$\begin{array}{l}\text { Semi-quantitative } \\
\text { cartilage defect } \\
\text { progression over } 30 \\
\text { months (WORMS) } \\
\text { (L) TFJ }\end{array}$} & Age, sex, race, BMl, & OR $(95 \%$ Cl) & OR $(95 \%$ Cl) & NA & Low (44) \\
\hline & & & & $\begin{array}{l}\text { Slow cartilage loss OR } \\
1.74 \text { (0.85 to } 3.55)\end{array}$ & $\begin{array}{l}\text { Slow cartilage loss OR } \\
1.79 \text { (0.83 to } 3.87)\end{array}$ & & \\
\hline & & & & $\begin{array}{l}\text { Fast cartilage loss OR } \\
1.32(0.37 \text { to } 4.78)\end{array}$ & $\begin{array}{l}\text { Fast cartilage loss OR } \\
1.0(0.24 \text { to } 4.10)\end{array}$ & & \\
\hline Kubota 2010 [92] & $\begin{array}{l}\text { MRI BML semi-quantitative } \\
\text { volume score change over }\end{array}$ & $\begin{array}{l}\mathrm{KL} \text { grade progression } \\
\text { over } 6 \text { months }(\mathrm{L}) \mathrm{TFJ}\end{array}$ & $\mathrm{Nil}$ & $\begin{array}{l}\text { BML score higher in } \mathrm{KL} \\
\text { progression group }\end{array}$ & NR & $\mathrm{NC}$ & Low (39) \\
\hline & o montus (L) IrJ & & & $p=0.044$ & & & \\
\hline Driban 2012 & MRI BML volume change & Change in cartilage & Nil & Cartilage thickness & NR & + & Low (28) \\
\hline anstiale $[00]$ & 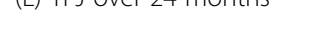 & denuded area of bone & & $r=-0.34, p=0.04$ & & & \\
\hline & & (L) TFJ over 24 months & & denuded bone & & & \\
\hline & & & & $r=0.42, p=0.01$ & & & \\
\hline
\end{tabular}


Table 1 Knee structural associations by feature and quality grade (Continued)

\begin{tabular}{|c|c|c|c|c|c|c|c|}
\hline & & & & $\begin{array}{l}\text { Femoral cartilage indices } \\
p>0.05\end{array}$ & & & \\
\hline Carrino 2006 [77] & $\begin{array}{l}\text { Crude presence of MRI } \\
\text { BML, TFJ (C) and (L) }\end{array}$ & $\begin{array}{l}\text { Any grade of cartilage } \\
\text { defect TFJ (C) and (L) }\end{array}$ & Nil & NR & NR & + & Low (22) \\
\hline \multicolumn{8}{|c|}{ MRI bone marrow lesion - cross-sectional studies } \\
\hline \multirow[t]{8}{*}{ Baranyay 2007 [63] } & \multirow{8}{*}{$\begin{array}{l}\text { MRI BML defined as large } \\
\text { or not large/absent in the } \\
\text { medial and lateral } \\
\text { compartments of TFJ (C) }\end{array}$} & \multirow{3}{*}{$\begin{array}{l}\text { MRI semi-quantitative } \\
\text { cartilage defects of } \\
\text { medial and lateral } \\
\text { compartments of } \\
\text { TFJ (C) }\end{array}$} & \multirow{8}{*}{$\begin{array}{l}\text { Age, gender, BMl, cartilage } \\
\text { volume or bone area }\end{array}$} & OR $(95 \% \mathrm{Cl})$ & OR $(95 \% \mathrm{Cl})$ & + & \multirow{8}{*}{$\begin{array}{l}\text { High } \\
(71 \%)\end{array}$} \\
\hline & & & & $\begin{array}{l}\text { Cartilage defect Medial } \\
\text { TFJ }\end{array}$ & $\begin{array}{l}\text { Cartilage defect Medial } \\
\text { TFJ }\end{array}$ & Cartilage defects & \\
\hline & & & & $1.81(1.26$ to 2.59$)$ & 1.80 (1.21 to 2.69$)$ & NA & \\
\hline & & \multirow{5}{*}{$\begin{array}{l}\text { Quantitative cartilage } \\
\text { volume medial and } \\
\text { lateral TFJ (C) }\end{array}$} & & $p=0.005$ & $p=0.004$ & & \\
\hline & & & & Lateral TFJ & Lateral TFJ & \multirow[t]{4}{*}{ Cartilage volume } & \\
\hline & & & & $1.52(1.14$ to 2.04$)$ & 1.45 (1.02 to 2.07$)$ & & \\
\hline & & & & $p=0.005$ & $p=0.04$ & & \\
\hline & & & & $\begin{array}{l}\text { No association with } \\
\text { ipsicompartmental } \\
\text { cartilage volume }\end{array}$ & $\begin{array}{l}\text { No association with } \\
\text { ipsicompartmental } \\
\text { cartilage volume }\end{array}$ & & \\
\hline \multirow[t]{7}{*}{ Guymer 2007 [35] } & \multirow{3}{*}{$\begin{array}{l}\text { Presence or absence of } \\
\text { MRI BMLs }\end{array}$} & \multirow{3}{*}{$\begin{array}{l}\text { Presence or absence } \\
\text { of semi-quantitative } \\
\text { cartilage defects }\end{array}$} & \multirow{7}{*}{$\begin{array}{l}\text { Age, height, weight, and } \\
\text { tibial cartilage volume }\end{array}$} & OR $(95 \% \mathrm{Cl})$ & OR $(95 \% \mathrm{Cl})$ & + & High (71) \\
\hline & & & & Medial TFJ & Medial TFJ & \multirow{6}{*}{$\begin{array}{l}\text { A positive association } \\
\text { is observed in the } \\
\text { medial but not the } \\
\text { lateral TFJ }\end{array}$} & \\
\hline & & & & 6.46 (1.04 to 38.39$)$ & 3.51 (1.08 to 11.42$)$ & & \\
\hline & \multirow{4}{*}{ (C) TFJ } & \multirow{4}{*}{ (C) TFJ } & & $p=0.04$ & $p=0.04$ & & \\
\hline & & & & Lateral TFJ & Lateral TFJ & & \\
\hline & & & & $1.17(0.22$ to 6.26$)$ & $1.02(0.17$ to 6.12$)$ & & \\
\hline & & & & $p=0.85$ & $p=0.98$ & & \\
\hline Stehling 2010 [65] & $\begin{array}{l}\text { Presence of any MRI semi- } \\
\text { quantitative BMLs (C) }\end{array}$ & $\begin{array}{l}\text { Presence of any } \\
\text { WORMS MRI cartilage } \\
\text { defects (C) }\end{array}$ & $\begin{array}{l}\text { Age, gender and BMI, KL } \\
\text { score, knee injury or knee } \\
\text { surgery, family history of } \\
\text { TKR and Heberden's nodes }\end{array}$ & $N R$ & $p<0.0001$ & + & High (71) \\
\hline Torres 2006 [103] & $\begin{array}{l}\text { MRI BML (WORMS) (C) } \\
\text { TFJ and PFJ }\end{array}$ & $\begin{array}{l}\text { Semi-quantitative } \\
\text { cartilage (WORMS) (C) }\end{array}$ & $\mathrm{Nil}$ & $R=0.56$ & NR & + & High (68) \\
\hline Ip 2011 [99] & $\begin{array}{l}\text { Semi-quantitative MRI } \\
\text { BML (C) }\end{array}$ & $\mathrm{KL}$ grade $(\mathrm{C})$ & $\begin{array}{l}\text { Age, sex, BMl, OA stage, } \\
\text { joint effusion, and meniscal } \\
\text { damage }\end{array}$ & $N R$ & $\begin{array}{l}\text { Highest BML score } \\
p<0.001\end{array}$ & + & High (68) \\
\hline Hayes 2005 [22] & $\begin{array}{l}\text { Semi-quantitative MRI } \\
\text { BML (C) }\end{array}$ & $\mathrm{KL}$ grade $(\mathrm{C})$ & $\mathrm{Nil}$ & $p=0.005$ & $N R$ & + & High (61) \\
\hline
\end{tabular}


Table 1 Knee structural associations by feature and quality grade (Continued)

\begin{tabular}{|c|c|c|c|c|c|c|c|}
\hline \multirow[t]{5}{*}{ Kornaat 2005 [100] } & \multirow{2}{*}{$\begin{array}{l}\text { Semi-quantitative MRI } \\
\text { BML (KOSS) }\end{array}$} & \multirow{5}{*}{$\begin{array}{l}\text { Semi-quantitative } \\
\text { cartilage defects } \\
\text { (KOSS) TFJ and PFJ (C) }\end{array}$} & \multirow[t]{5}{*}{$\mathrm{Nil}$} & OR $(95 \%$ Cl) & \multirow[t]{5}{*}{ NR } & \multirow[t]{5}{*}{+} & \multirow[t]{5}{*}{ Low (57) } \\
\hline & & & & PFJ & & & \\
\hline & \multirow[t]{3}{*}{ TFJ and PFJ (C) } & & & 17 (3.8 to 72 ) & & & \\
\hline & & & & TFJ & & & \\
\hline & & & & $120(6.5$ to 2,221$)$ & & & \\
\hline \multirow{3}{*}{$\begin{array}{l}\text { Gudbergsen } 2013 \\
\text { [98] }\end{array}$} & \multirow{3}{*}{$\begin{array}{l}\text { Semi-quantitative MRI } \\
\text { BML (BLOKS) (C) }\end{array}$} & \multirow[t]{3}{*}{$\mathrm{KL}$ grade $(\mathrm{C})$} & \multirow[t]{3}{*}{$\mathrm{Nil}$} & $\mathrm{KL}$ grade & \multirow[t]{3}{*}{ NR } & \multirow[t]{3}{*}{+} & \multirow[t]{3}{*}{ Low (57) } \\
\hline & & & & $p=0.046$ lateral & & & \\
\hline & & & & $p<0.001$ medial & & & \\
\hline Link 2003 [101] & $\begin{array}{l}\text { Semi-quantitative MRI } \\
\text { BML, (C) }\end{array}$ & $\mathrm{KL}$ grade $(\mathrm{C})$ & Nil & $p<0.05$ & NR & + & Low (54) \\
\hline \multirow[t]{2}{*}{ Sowers 2003 [29] } & \multirow{2}{*}{$\begin{array}{l}\text { Semi-quantitative MRI } \\
\text { BML (C) }\end{array}$} & \multirow{2}{*}{$\begin{array}{l}\text { Semi-quantitative } \\
\text { cartilage defect (C) }\end{array}$} & \multirow[t]{2}{*}{$\mathrm{Nil}$} & $p$ for trend & \multirow[t]{2}{*}{ NR } & \multirow[t]{2}{*}{+} & \multirow[t]{2}{*}{ Low (54) } \\
\hline & & & & $p<0.0001$ & & & \\
\hline Felson 2001 [96] & $\begin{array}{l}\text { Semi-quantitative MRI } \\
\text { BMLs (C) }\end{array}$ & $\mathrm{KL}$ grade $(\mathrm{C})$ & Nil & $N R$ & NR & + & Low (54) \\
\hline Lo 2005 [102] & $\begin{array}{l}\text { Semi-quantitative MRI } \\
\text { BML (WORMS } \geq 1 \text { ) (C) }\end{array}$ & $\mathrm{KL}$ grade $\geq 2(\mathrm{C})$ & Nil & NR & NR & + & Low (50) \\
\hline \multirow[t]{2}{*}{ Meredith 2009 [64] } & $\begin{array}{l}\text { Sum of semi-quantitative } \\
\text { MRI }\end{array}$ & $\begin{array}{l}\text { Sum of semi- } \\
\text { quantitative MRI }\end{array}$ & \multirow[t]{2}{*}{$\mathrm{Nil}$} & \multirow[t]{2}{*}{$p<0.0003$} & \multirow[t]{2}{*}{ NR } & \multirow[t]{2}{*}{+} & \multirow[t]{2}{*}{ Low (50) } \\
\hline & $\begin{array}{l}\text { BML scores in the TFJ } \\
\text { and PFJ (C) }\end{array}$ & $\begin{array}{l}\text { Cartilage defect scores } \\
\text { in the TFJ and PFJ (C) }\end{array}$ & & & & & \\
\hline $\begin{array}{l}\text { Fernandez-Madrid } \\
1994 \text { [97] }\end{array}$ & $\begin{array}{l}\text { Crude presence of MRI } \\
\text { BMLs (C) }\end{array}$ & $\mathrm{KL}$ grade $(\mathrm{C})$ & Nil & $p<0.001$ & NR & + & Low (46) \\
\hline Scher 2008 [87] & $\begin{array}{l}\text { Semi-quantitative MRI } \\
\text { BML (C) }\end{array}$ & $\begin{array}{l}\text { Semi-quantitative } \\
\text { cartilage defect } \\
\text { (modified Noyes) (C) }\end{array}$ & Nil & $p=0.012$ & NR & + & Low (43) \\
\hline \multicolumn{8}{|c|}{ MRI bone marrow lesion - case control studies } \\
\hline \multirow[t]{7}{*}{ Ratzlaff 2014 [104] } & \multirow{7}{*}{$\begin{array}{l}\text { Total tibial BML volume } 12 \\
\text { and } 24 \text { months before TKR } \\
\text { and interval change } \\
\text { between } 12 \text { and } 24 \text { (C) } \\
\text { and (L) TFJ }\end{array}$} & \multirow[t]{7}{*}{ Incident TKR (L) } & NB matched cases and & OR $(95 \% \mathrm{Cl})$ & NR & + & High (65) \\
\hline & & & & 12 months $(C)$ & & True of TFJ but not & \\
\hline & & & & 1.68 (1.33 to 2.13$)$ & & & \\
\hline & & & & 24 months $(C)$ & & & \\
\hline & & & & 1.35 (1.02 to 1.78$)$ & & & \\
\hline & & & & $\begin{array}{l}12 \text { to } 24 \text { months } \\
\text { change }(L)\end{array}$ & & & \\
\hline & & & & $1.23(1.03$ to 1.46$)$ & & & \\
\hline Zhao 2010 [105] & $\begin{array}{l}\text { Baseline crude presence } \\
\text { of MRI BMLs at (C) TFJ }\end{array}$ & $\begin{array}{l}\text { Overlying cartilage } \\
\text { defect progression } \\
\text { after } 1 \text { year (WORMS) } \\
\text { (L) TFJ }\end{array}$ & Nil & $\begin{array}{l}\text { Change in cartilage } \\
\text { defect scores for areas } \\
\text { with and without } \\
\text { underlying BMLs }\end{array}$ & NR & + & Low (56) \\
\hline
\end{tabular}


Table 1 Knee structural associations by feature and quality grade (Continued)

\begin{tabular}{|c|c|c|c|c|c|c|c|}
\hline & & & & $p=0.00003$ & & & \\
\hline \multirow{3}{*}{$\begin{array}{l}\text { Aitken } 2013 \\
\text { Abstract [17] }\end{array}$} & \multirow{3}{*}{$\begin{array}{l}\text { Semi-quantitative BMLs } \\
\text { tibia, femur and patella }\end{array}$} & \multirow{3}{*}{$\begin{array}{l}\text { Cartilage volume and } \\
\text { defect score tibia and } \\
\text { femur }\end{array}$} & \multirow[t]{3}{*}{ Age, sex, BMl } & \multirow[t]{3}{*}{ NR } & Tibial cartilage volume & \multirow[t]{3}{*}{ - } & \multirow[t]{3}{*}{ Low (47) } \\
\hline & & & & & $\begin{array}{l}\beta=-433 \mathrm{~mm}^{3} \text { per } \\
\text { unit increase in BML }\end{array}$ & & \\
\hline & & & & & $p<0.01$ & & \\
\hline Stahl 2011 [41] & $\begin{array}{l}\text { Semi-quantitative MRI BML } \\
\text { size (WORMS) (L) TFJ }\end{array}$ & $\begin{array}{l}\text { Semi-quantitative } \\
\text { cartilage defect size } \\
\text { (L) TFJ }\end{array}$ & Nil & NR & $p<0.165$ & NA & Low (47) \\
\hline \multicolumn{8}{|c|}{ MRI osteophyte - cohort studies } \\
\hline \multirow{2}{*}{$\begin{array}{l}\text { De-Lange } 2014 \\
\text { abstract [106] }\end{array}$} & \multirow{2}{*}{$\begin{array}{l}\text { Semi-quantitative } \\
\text { osteophyte (KOSS) (C) }\end{array}$} & \multirow{2}{*}{$\begin{array}{l}\text { Radiographic } \\
\text { progression of JSN } \\
\text { of TFJ (L) }\end{array}$} & \multirow{2}{*}{$\begin{array}{l}\text { Age, gender, BMI and } \\
\text { baseline JSN }\end{array}$} & \multirow[t]{2}{*}{ NR } & OR $(95 \% \mathrm{Cl})$ & + & \multirow[t]{2}{*}{ High (61) } \\
\hline & & & & & $1.8(1.1$ to 3.1$)$ & $\begin{array}{l}\text { Higher OST score, } \\
\text { the higher the JSN }\end{array}$ & \\
\hline $\begin{array}{l}\text { Liu } 2014 \\
\text { Abstract [45] }\end{array}$ & $\begin{array}{l}\text { Baseline semi-quantitative } \\
\text { osteophyte score (WORMS) } \\
\text { (C) TFJ }\end{array}$ & $\begin{array}{l}\text { Incident TKR at } \\
6 \text {-months follow } \\
\text { up }(L)\end{array}$ & $\begin{array}{l}\text { Activity of daily living } \\
\text { disability score }\end{array}$ & NR & $\begin{array}{l}\mathrm{RR}(95 \% \mathrm{Cl}) 3.01(1.39 \\
\text { to } 6.52)\end{array}$ & + & Low (50) \\
\hline \multirow[t]{2}{*}{ Sowers 2011 [28] } & \multirow[t]{2}{*}{$\begin{array}{l}\text { Semi-quantitative MRI } \\
\text { osteophyte size in TFJ (C) }\end{array}$} & \multirow{2}{*}{$\begin{array}{l}\text { Progression in } \mathrm{KL} \\
\text { grade (11-year follow } \\
\text { up) }(\mathrm{L})\end{array}$} & \multirow[t]{2}{*}{$\mathrm{Nil}$} & $\begin{array}{l}R(95 \% \text { Cl) medial tibia } \\
0.65(0.59 \text { to } 0.71)\end{array}$ & \multirow[t]{2}{*}{ NR } & \multirow[t]{2}{*}{+} & \multirow[t]{2}{*}{ Low (53) } \\
\hline & & & & $\begin{array}{l}\text { Lateral tibia } \sim 0.57(0.49 \\
\text { to } 0.63)\end{array}$ & & & \\
\hline \multicolumn{8}{|c|}{ MRI osteophyte - cross-sectional studies } \\
\hline Stehling 2010 [65] & $\begin{array}{l}\text { Presence of any MRI semi- } \\
\text { quantitative osteophytes (C) }\end{array}$ & $\begin{array}{l}\text { Presence of any } \\
\text { WORMS MRI cartilage } \\
\text { defects (C) }\end{array}$ & $\begin{array}{l}\text { Age, gender and BMI, KL } \\
\text { score, knee injury or knee } \\
\text { surgery, family history of } \\
\text { TKR and Heberden's nodes }\end{array}$ & NR & $p=0.0037$ & + & High (71) \\
\hline Torres 2006 [103] & $\begin{array}{l}\text { MRI osteophyte, (WORMS) } \\
\text { TFJ and PFJ (C) }\end{array}$ & $\begin{array}{l}\text { Semi-quantitative } \\
\text { cartilage (WORMS) } \\
\text { TFJ and PFJ (C) }\end{array}$ & $\mathrm{Nil}$ & $R=0.73$ & $N R$ & + & High (68) \\
\hline Hayes 2005 [22] & $\begin{array}{l}\text { Semi-quantitative MRI } \\
\text { osteophyte }(C)\end{array}$ & $\mathrm{KL}$ grade $(\mathrm{C})$ & $\mathrm{Nil}$ & $p<0.001$ & $N R$ & + & High (61) \\
\hline \multirow[t]{2}{*}{ Meredith 2009 [64] } & $\begin{array}{l}\text { Sum of semi-quantitative } \\
\text { MRI }\end{array}$ & $\begin{array}{l}\text { Sum of semi- } \\
\text { quantitative MRI }\end{array}$ & \multirow[t]{2}{*}{ Nil } & \multirow[t]{2}{*}{$p<0.0001$} & \multirow[t]{2}{*}{ NR } & \multirow[t]{2}{*}{+} & \multirow[t]{2}{*}{ Low (50) } \\
\hline & $\begin{array}{l}\text { Osteophyte scores in the } \\
\text { TFJ and PFJ (C) }\end{array}$ & $\begin{array}{l}\text { cartilage defect scores } \\
\text { in the TFJ and PFJ (C) }\end{array}$ & & & & & \\
\hline \multirow[t]{2}{*}{ McCauley 2001 [26] } & \multirow{2}{*}{$\begin{array}{l}\text { MRI central osteophyte } \\
\text { presence (C) TFJ }\end{array}$} & \multirow{2}{*}{$\begin{array}{l}\text { MRI cartilage lesion } \\
\text { presence (C) TFJ }\end{array}$} & \multirow[t]{2}{*}{ Nil } & \multirow{2}{*}{$\begin{array}{l}\text { Crude association of } 32 \\
\text { of } 35 \text { central osteophytes } \\
\text { having adjacent cartilage } \\
\text { lesions }\end{array}$} & \multirow[t]{2}{*}{ NR } & + & Low (29) \\
\hline & & & & & & Crude, unadjusted & \\
\hline Roemer 2012 [108] & MRI osteophyte & Cartilage defect & Age, sex, BMI, race, TFJ & OR 2378.1, & OR 108.8, & + & Low (57) \\
\hline & (WORMS) (C) & & & $95 \%$ Cl 249.8 to 22643.4 & $95 \% \mathrm{Cl} 14.2$ to 834.9 & & \\
\hline & & & & & $p$ for trend $<0.0001$ & & \\
\hline
\end{tabular}


Table 1 Knee structural associations by feature and quality grade (Continued)

\begin{tabular}{|c|c|c|c|c|c|c|c|}
\hline Link 2003 [101] & $\begin{array}{l}\text { Semi-quantitative MRI } \\
\text { osteophytes (C) }\end{array}$ & $\mathrm{KL}$ grade $(\mathrm{C})$ & $\mathrm{Nil}$ & $p<0.01$ & $N R$ & + & Low (54) \\
\hline $\begin{array}{l}\text { Fernandez-Madrid } \\
1994 \text { [97] }\end{array}$ & $\begin{array}{l}\text { Crude presence of MRI } \\
\text { osteophytes (C) }\end{array}$ & $\mathrm{KL}$ grade $(\mathrm{C})$ & $\mathrm{Nil}$ & $p<0.001$ & NR & + & Low (46) \\
\hline \multicolumn{8}{|c|}{ MRI bone attrition - cohort studies } \\
\hline \multirow[t]{2}{*}{ Kothari 2010 [82] } & Semi-quantitative baseline & \multirow{2}{*}{$\begin{array}{l}\text { Semi-quantitative } \\
\text { cartilage defect score } \\
\text { change over } 2 \text { years } \\
\text { (WORMS) (L) TFJ. }\end{array}$} & \multirow{2}{*}{$\begin{array}{l}\text { Age, sex BMl, other bone } \\
\text { lesions }\end{array}$} & OR 3.17, & OR 1.85, & \multirow[t]{2}{*}{ NA } & \multirow[t]{2}{*}{ Low (50) } \\
\hline & (WORMS) (C) TFJ & & & $95 \% \mathrm{Cl} 1.64$ to 6.16 & $95 \% \mathrm{Cl} 0.71$ to 4.82 & & \\
\hline \multicolumn{8}{|c|}{ MRI bone attrition - cross-sectional studies } \\
\hline Torres 2006 [103] & $\begin{array}{l}\text { MRI attrition (WORMS) TFJ } \\
\text { and PFJ (C) }\end{array}$ & $\begin{array}{l}\text { Semi-quantitative } \\
\text { cartilage (WORMS) } \\
\text { TFJ and PFJ (C) }\end{array}$ & $\mathrm{Nil}$ & $R=0.75$ & NR & + & High (68) \\
\hline \multirow{2}{*}{$\begin{array}{l}\text { Reichenbach } \\
2008 \text { [110] }\end{array}$} & \multirow{2}{*}{$\begin{array}{l}\text { Semi-quantitative MRI bone } \\
\text { attrition (WORMS) (C) }\end{array}$} & \multirow{2}{*}{$\begin{array}{l}\mathrm{KL} \text { grade and semi- } \\
\text { quantitative cartilage } \\
\text { defects (WORMS) (C) }\end{array}$} & \multirow[t]{2}{*}{ Nil } & NR & \multirow[t]{2}{*}{ NR } & \multirow[t]{2}{*}{+} & \multirow[t]{2}{*}{ Low (43) } \\
\hline & & & & Crude correlation & & & \\
\hline \multicolumn{8}{|c|}{ MRI bone attrition - case control studies } \\
\hline \multirow[t]{2}{*}{ Neogi 2009 [109] } & \multirow{2}{*}{$\begin{array}{l}\text { Baseline semi-quantitative } \\
\text { MRI bone attrition size } \\
\text { (WORMS) (C) TFJ }\end{array}$} & \multirow{2}{*}{$\begin{array}{l}\text { Cartilage defects } \\
\text { progression (WORMS) } \\
\text { after } 30 \text { months TFJ }\end{array}$} & \multirow[t]{2}{*}{ Age, sex, BMl } & OR 5.5, & OR 3.0, & \multirow[t]{2}{*}{+} & \multirow[t]{2}{*}{ Low (59) } \\
\hline & & & & $95 \%$ Cl 3.0 to 10.0 & $95 \% \mathrm{Cl} 2.2$ to 4.2 & & \\
\hline \multicolumn{8}{|c|}{ MRI bone Shape/dimension - cohort studies } \\
\hline \multirow[t]{3}{*}{ Cicuttini 2004 [111] } & \multirow{3}{*}{$\begin{array}{l}\text { Baseline quantitative MRI } \\
\text { tibial bone area }(C)\end{array}$} & \multirow{3}{*}{$\begin{array}{l}\text { TKR incidence }(L) \text { over } \\
4 \text { years }\end{array}$} & \multirow{3}{*}{$\begin{array}{l}\text { Age, sex, height, weight, } \\
\text { BMI, WOMAC, ROA severity }\end{array}$} & \multirow[t]{3}{*}{ NR } & OR $(95 \% \mathrm{Cl})$ & \multirow[t]{3}{*}{+} & \multirow[t]{3}{*}{ High (78) } \\
\hline & & & & & $1.2(1.0$ to 1.4$)$ & & \\
\hline & & & & & $p=0.02$ & & \\
\hline \multirow[t]{6}{*}{ Ding 2008 [20] } & \multirow{6}{*}{$\begin{array}{l}\text { Baseline MRI tibial bone } \\
\text { area (C) TFJ }\end{array}$} & Progressive cartilage & Age, sex, BMI, OA family & $\beta(95 \% \mathrm{Cl})$ & $\beta(95 \% \mathrm{Cl})$ & - & High (72) \\
\hline & & volume loss (L) IFJ & $\begin{array}{l}\text { history, muscle strength } \\
\text { and ROA. }\end{array}$ & Medial femoral cartilage & Medial femoral cartilage & & \\
\hline & & & & $\beta=0.17$ (0.04 to 0.29 ) & $\beta=0.35$ (0.14 to 0.56$)$ & & \\
\hline & & & & Total femoral cartilage & Total femoral cartilage & & \\
\hline & & & & $\beta=0.07$ & $\beta=0.13$ & & \\
\hline & & & & (0.003 to 0.14$)$ & (0.02 to 0.25 ) & & \\
\hline Ding 2006 [18] & Baseline MRI tibial bone & Change in semi- & Age, sex, BMl, radiographic & NA & OR $(95 \% \mathrm{Cl})$ & - & High (61) \\
\hline & & $\begin{array}{l}\text { quantitative MIRI } \\
\text { cartilage defect scores }\end{array}$ & OA \feature & & Medial TFJ & & \\
\hline & & over 2.3 years $(L)$ TFJ & & & $1.24(1.01$ to 1.51$)$ & & \\
\hline & & & & & $p=0.04$ & & \\
\hline & & & & & Lateral TFJ & & \\
\hline & & & & & 2.07 (1.52 to 2.82$)$ & & \\
\hline & & & & & $p<0.001$ & & \\
\hline
\end{tabular}


Table 1 Knee structural associations by feature and quality grade (Continued)

\begin{tabular}{|c|c|c|c|c|c|c|c|}
\hline \multirow[t]{6}{*}{ Everhart 2014 [114] } & \multirow{6}{*}{$\begin{array}{l}\text { Baseline TFJ subchondral } \\
\text { surface ratio of medial and } \\
\text { lateral TFJ compartments } \\
\text { (C) }\end{array}$} & \multirow{6}{*}{$\begin{array}{l}\text { Radiographic } \\
\text { progression of lateral } \\
\text { or medial TFJ knee } \\
\text { OA at } 48 \text { months (L) }\end{array}$} & \multirow{6}{*}{$\begin{array}{l}\text { Sex, race, age, BMI, tobacco } \\
\text { use, activity level, knee } \\
\text { coronal alignment, baseline } \\
\text { symptoms, injury history, } \\
\text { surgery history, KL grade, } \\
\text { and JSW }\end{array}$} & $\begin{array}{l}\text { Unadjusted medial SSR } \\
\text { vs progression of medial } \\
\text { JSN }\end{array}$ & \multirow{6}{*}{$\begin{array}{l}\text { Neither medial nor } \\
\text { lateral SSR was } \\
\text { associated lateral } \\
\text { or medial ROA } \\
\text { progression in } \\
\text { adjusted analysis } \\
p<0.05 \text {. }\end{array}$} & \multirow[t]{6}{*}{ NA } & \multirow[t]{6}{*}{ High (61) } \\
\hline & & & & $\begin{array}{l}\text { OR } 1.43,95 \% \mathrm{Cl} 1.15 \\
\text { to } 1.77\end{array}$ & & & \\
\hline & & & & $p=0.0015$ & & & \\
\hline & & & & $\begin{array}{l}\text { Medial SSR vs progression } \\
\text { of lateral JSN }\end{array}$ & & & \\
\hline & & & & $\begin{array}{l}\text { OR } 1.87,95 \% \mathrm{Cl} 1.44 \\
\text { to } 2.42\end{array}$ & & & \\
\hline & & & & $p<0.001$ & & & \\
\hline \multirow{2}{*}{$\begin{array}{l}\text { Davies-Tuck } \\
2008 \text { [112] }\end{array}$} & \multirow{2}{*}{$\begin{array}{l}\text { Baseline MRI tibial bone } \\
\text { plateau area (C) TFJ }\end{array}$} & \multirow{2}{*}{$\begin{array}{l}\text { Progressive semi- } \\
\text { quantitative cartilage } \\
\text { defect score }(L) \text { medial } \\
\text { and lateral TFJ }\end{array}$} & \multirow{2}{*}{$\begin{array}{l}\text { Age, sex, BMI, baseline } \\
\text { cartilage defect score, } \\
\text { baseline cartilage volume } \\
\text { and baseline tibial plateau } \\
\text { area }\end{array}$} & Lateral TFJ & OR $(95 \% \mathrm{Cl})$ & \multirow[t]{2}{*}{+} & \multirow[t]{2}{*}{ High (56 } \\
\hline & & & & $\begin{array}{l}\text { OR }(95 \% \mathrm{Cl})-0.01(-0.06 \\
\text { to } 0.03) p=0.59\end{array}$ & $\begin{array}{l}\text { Lateral TFJ } 0.06(0.004 \\
\text { to } 0.11) p=0.03 \\
\text { Medial TFJ } 0.07(0.03 \\
\text { to } 0.12) p=0.002\end{array}$ & & \\
\hline Carnes 2012 [113] & MRI tibial bone area $(C)$ & $\begin{array}{l}\text { Semi-quantitative } \\
\text { cartilage defect } \\
\text { progression TFJ (L) }\end{array}$ & $\begin{array}{l}\text { Age, sex, BMI, cartilage } \\
\text { defects, BML }\end{array}$ & $\begin{array}{l}\text { Lateral tibial bone area OR } \\
1.11,95 \% \mathrm{Cl} 1.0 \text { to } 1.23\end{array}$ & $\begin{array}{l}\text { OR }(95 \% \text { CI) bone } \\
\text { area medial } 1.12(1.01 \\
\text { to } 1.26) \text { and lateral } \\
\text { tibial }(1.35(1.12 \text { to } \\
1.63)\end{array}$ & + & Low (50) \\
\hline Dore 2010 [68] & $\begin{array}{l}\text { Baseline tibial bone area } \\
\text { MRI (C) }\end{array}$ & $\begin{array}{l}\text { Increase or no increase } \\
\text { in semi-quantitative } \\
\text { MRI tibial cartilage } \\
\text { defects over } 2.7 \text { years } \\
(\mathrm{L})\end{array}$ & $\begin{array}{l}\text { Age, sex, body mass index, } \\
\text { baseline cartilage defects, } \\
\text { and subchondral bone } \\
\text { mineral density }\end{array}$ & NR & $\begin{array}{l}\text { OR }(95 \% \mathrm{Cl}) \text { medial } \\
\text { tibia } 1.6(1.0 \text { to } 2.6) \\
p=0.04 \text { lateral tibia } \\
2.4(1.4 \text { to } 4.0) p<0.01\end{array}$ & $\begin{array}{l}\text { + Bone area size is } \\
\text { associated with } \\
\text { increasing cartilage } \\
\text { defect scores }\end{array}$ & Low (50) \\
\hline $\begin{array}{l}\text { Hudelmaier } 2013 \\
\text { [180] Abstract }\end{array}$ & $\begin{array}{l}\text { Annual change in } \\
\text { segmented MRI knee } \\
\text { bone area }(L)\end{array}$ & Baseline $\mathrm{KL}$ grade $(\mathrm{C})$ & Nil & Medial tibia $p<0.05$ & NR & $\begin{array}{l}\text { + The higher the } \mathrm{KL} \\
\text { grade the larger the } \\
\text { increase in bone area }\end{array}$ & Low (50) \\
\hline \multicolumn{8}{|c|}{ MRI bone shape/dimension - cross-sectional studies } \\
\hline Ding 2005 [19] & $\begin{array}{l}\text { MRI quantitative tibial } \\
\text { bone area }(\mathrm{C})\end{array}$ & $\begin{array}{l}\text { Semi-quantitative MRI } \\
\text { knee cartilage defect } \\
\text { severity scores (C) TFJ }\end{array}$ & $\begin{array}{l}\text { Age, sex, BMl, family history, } \\
\text { cartilage volume }\end{array}$ & $\begin{array}{l}\beta(95 \% \text { Cl) medial TFJ } \\
0.06(0.03 \text { to } 0.09) \text { lateral } \\
\text { TFJ } 0.09 \text { ( } 0.05 \text { to } 0.13)\end{array}$ & $\begin{array}{l}\beta(95 \% \mathrm{Cl}) \text { medial TFJ } \\
0.11(0.07 \text { to } 0.15) \\
\text { lateral TFJ } 0.17(0.11 \\
\text { to } 0.22)\end{array}$ & $\begin{array}{l}\text { + Association } \\
\text { maintained for the } \\
\text { whole TFJ and by } \\
\text { compartment }\end{array}$ & High (64) \\
\hline $\begin{array}{l}\text { Kalichman } \\
2007[165]\end{array}$ & $\begin{array}{l}\text { MRI patellar length ratio, } \\
\text { trochlea sulcus angle (C) }\end{array}$ & JSN grade $(C)$ & Age, sex, BMl & NR & $\begin{array}{l}\text { Trochlea sulcus angle } \\
p \text { for trend, medial } \\
\text { JSN } p=0.0162 \text {, lateral } \\
\text { JSN } p=0.1206\end{array}$ & NC & High (64) \\
\hline $\begin{array}{l}\text { Kalichman } \\
2007[115]\end{array}$ & $\begin{array}{l}\text { MRI patellar length ratio, } \\
\text { trochlea sulcus angle }(C)\end{array}$ & $\begin{array}{l}\text { Cartilage defect } \\
\text { (WORMS) (C) }\end{array}$ & Age, sex, BMl & NR & $\begin{array}{l}\text { Trochlea sulcus angle } \\
p \text { for trend, medial } \\
\text { cartilage loss } p= \\
0.0016 \text {, lateral cartilage } \\
\text { loss } p=0.0009\end{array}$ & + & Low (57) \\
\hline
\end{tabular}


Table 1 Knee structural associations by feature and quality grade (Continued)

\begin{tabular}{|c|c|c|c|c|c|c|c|}
\hline Stefanik 2012 [116] & $\begin{array}{l}\text { MRI lateral trochlear } \\
\text { inclination and trochlear } \\
\text { angle (C) }\end{array}$ & $\begin{array}{l}\text { Semi-quantitative } \\
\text { cartilage defect } \\
\text { (WORMS) (C) }\end{array}$ & Age, sex, BMl & NR & $\begin{array}{l}\text { Lateral trochlear } \\
\text { inclination OR 2, } \\
95 \% \mathrm{Cl} 1.9 \text { to } 3.7 \\
p<0.0001 \text {, trochlear } \\
\text { angle } \mathrm{OR} 2.0,95 \% \mathrm{Cl} \\
1.2 \text { to } 3.5, p<0.0001\end{array}$ & + & Low (57) \\
\hline Frobell 2010 [107] & $\begin{array}{l}\text { MRI bone area - manual } \\
\text { segmentation }(C)\end{array}$ & $\begin{array}{l}\text { KL grade, OARSI JSN } \\
\text { grade }(C)\end{array}$ & Age and BMl & $\begin{array}{l}\text { Medial tibia JSN and KL } \\
\mathrm{p}<0.0125\end{array}$ & $\begin{array}{l}\text { Medial tibia JSN and } \\
\mathrm{KL} p<0.0125\end{array}$ & + & Low (57) \\
\hline Wang 2005 [66] & $\begin{array}{l}\text { Annual \% change in tibial } \\
\text { bone area }(L) 2 \text { years } \\
\text { follow up }\end{array}$ & Baseline JSN (C) & $\begin{array}{l}\text { Age, sex, BMI, WOMAC } \\
\text { score, SF-36 score, physical } \\
\text { activity, radiographic OA } \\
\text { features, baseline tibial } \\
\text { plateau bone area. }\end{array}$ & $\begin{array}{l}\beta(95 \% \text { Cl) medial tibia } \\
\beta=0.35(-1.10 \text { to } 1.80) \\
p=0.63 \text {, lateral tibia } \\
-0.87(-2.35 \text { to } 0.61) \\
p=0.25\end{array}$ & $\begin{array}{l}\beta(95 \% \mathrm{Cl}) \text { medial } \\
\text { tibia } 1.88 \text { (0.43 to } 3.33) \\
p=0.01 \text { lateral tibia } \\
-0.42(-2.31 \text { to } 1.48) \\
p=0.66\end{array}$ & $\begin{array}{l}\text { + Association with } \\
\text { medial tibia but not } \\
\text { in the lateral tibia }\end{array}$ & Low (57) \\
\hline Jones 2004 [23] & Tibial bone area (MRI) (C) & Radiographic JSN (C) & Age, sex, height, weight & $\begin{array}{l}\beta(95 \% \mathrm{Cl}) \text { medial tibia } \\
\beta=-0.03(-0.11 \text { to } 0.06), \\
\text { lateral tibia }-0.00(-0.07 \\
\text { to } 0.06)\end{array}$ & $\begin{array}{l}\beta(95 \% \mathrm{Cl}) \text { medial } \\
\text { tibia } \beta=-0.00(-0.04 \\
\text { to } 0.06) \text {, lateral tibia } \\
+0.00(-0.04 \text { to } 0.05)\end{array}$ & NA & Low (50) \\
\hline Eckstein 2010 [117] & $\begin{array}{l}\text { MRI tibial bone area } \\
\text { (segmented) }(C)\end{array}$ & OARSI JSN grade $(C)$ & Nil & $p<0.01$ & NR & + & Low (43) \\
\hline \multicolumn{8}{|c|}{ MRI bone shape/dimension - case-control studies } \\
\hline Bowes 2013 [118] & $\begin{array}{l}\text { Change in segmented } \\
\text { MRI 3D bone area over } \\
4 \text { years }(L)\end{array}$ & $\begin{array}{l}\mathrm{KL} \text { grade defined } \mathrm{ROA} \\
\text { knee }(\mathrm{C}) \text { and }(\mathrm{L})\end{array}$ & Nil & $\begin{array}{l}\text { NR bone area increased } \\
\text { significantly faster in ROA } \\
\text { vs non-ROA } p<0.0001\end{array}$ & NR & $\begin{array}{l}\text { + Higher KL grades } \\
\text { had greater increase } \\
\text { in bone area, }\end{array}$ & High (71) \\
\hline Neogi 2013 [120] & $\begin{array}{l}\text { MRI 3D bone shape (tibia, } \\
\text { femur and patella) (C) }\end{array}$ & $\begin{array}{l}\text { Incident TFJ ROA KL } \\
\text { grade } \geq 2(\mathrm{~L})\end{array}$ & Age, sex, BMl & NR & OR $3,95 \%$ Cl 1.8 to 5.0 & $\begin{array}{l}\text { + Developing } 3 D \\
\text { OA knee shape is } \\
\text { associated with } \\
\text { increasing ROA knee }\end{array}$ & High (65) \\
\hline $\begin{array}{l}\text { Hunter } 2013 \\
\text { abstract [119] }\end{array}$ & $\begin{array}{l}\text { Change in MRI knee bone } \\
\text { area over } 24 \text { months }(L)\end{array}$ & $\begin{array}{l}\text { Incident TFJ ROA (KL } \\
\text { grade } \geq 2)(L)\end{array}$ & NR & NR & $\begin{array}{l}\text { Hazard ratio }(95 \% \mathrm{Cl}) \\
\text { range from } 1.17(1.08 \\
\text { to } 1.27) \text { to } 3.97(2.38 \\
\text { to } 6.63) \text {, all highly } \\
\text { statistically significant }\end{array}$ & $\begin{array}{l}+ \text { for all bone } \\
\text { regions Enlarging } \\
\text { bone area associated } \\
\text { with increasing ROA } \\
\text { knee }\end{array}$ & Low (59) \\
\hline Wluka 2005 [121] & $\begin{array}{l}\text { Change in MRI tibial bone } \\
\text { area }(\mathrm{L})\end{array}$ & $\begin{array}{l}\text { Baseline radiographic } \\
\text { JSN (C) }\end{array}$ & $\begin{array}{l}\text { Age, BMI, pain, physical } \\
\text { activity }\end{array}$ & $\begin{array}{l}\text { Medial tibial bone area } \\
R=160,95 \% \mathrm{Cl} 120 \text { to } \\
201, p<0.001\end{array}$ & $\begin{array}{l}\text { Medial tibial bone } \\
\text { area } R=145,95 \% \mathrm{Cl} \\
103 \text { to } 186, p<0.001\end{array}$ & + & Low (47) \\
\hline \multicolumn{8}{|c|}{ MRI bone cyst - cohort studies } \\
\hline Kotharii 2010 [82] & $\begin{array}{l}\text { Semi-quantitative baseline } \\
\text { MRI bone cyst (WORMS) } \\
\text { (C) TFJ }\end{array}$ & $\begin{array}{l}\text { Semi-quantitative } \\
\text { cartilage defect score } \\
\text { change over } 2 \text { years } \\
\text { (WORMS) (L) TFJ. }\end{array}$ & $\begin{array}{l}\text { Age, sex BMI, other bone } \\
\text { lesions }\end{array}$ & $\begin{array}{l}\text { OR } 1.66,95 \% \mathrm{Cl} 0.55 \\
\text { to } 4.99\end{array}$ & $\begin{array}{l}\text { OR } 0.47,95 \% \mathrm{Cl} 0.11 \\
\text { to } 2.03\end{array}$ & NA & Low (50) \\
\hline Tanamas 2010 [88] & $\begin{array}{l}\text { Semi-quantitative change } \\
\text { in MRI bone cyst size }(\mathrm{L})\end{array}$ & $\begin{array}{l}\text { Knee Cartilage volume } \\
\text { loss over } 2 \text { years (L) } \\
\text { TFJ }\end{array}$ & Nil & $\begin{array}{l}\beta(95 \% \mathrm{Cl}) \text { lateral tibial } \\
\text { cartilage loss in cyst } \\
\text { regression relative to } \\
\text { stable and progressive } \\
\text { cysts }\end{array}$ & NR & + & Low (47) \\
\hline
\end{tabular}


Table 1 Knee structural associations by feature and quality grade (Continued)

\begin{tabular}{|c|c|c|c|c|c|c|c|}
\hline & & & & $\begin{array}{l}\beta=-11.81(-16.64 \\
\text { to }-6.98)\end{array}$ & & & \\
\hline $\begin{array}{l}\text { Madan-Sharma } \\
2008 \text { [93] }\end{array}$ & $\begin{array}{l}\text { Baseline MRI semi- } \\
\text { quantitative bone cyst } \\
\text { (C) TFJ }\end{array}$ & $\begin{array}{l}\text { OARSI medial TFJ JSN } \\
\text { grade progression } \\
\text { over } 2 \text { years (L) TFJ }\end{array}$ & $\begin{array}{l}\text { Age, sex, BMI and family } \\
\text { effect }\end{array}$ & NR & $\begin{array}{l}R R 1.6,95 \% \mathrm{Cl} 0.5 \text { to } \\
4.0\end{array}$ & NA & Low (47) \\
\hline Carrino 2006 [77] & $\begin{array}{l}\text { Crude presence of MRI } \\
\text { bone cyst TFJ (C) and (L) }\end{array}$ & $\begin{array}{l}\text { Any grade of cartilage } \\
\text { defect TFJ (C) and (L) }\end{array}$ & Nil & NR & NR & + & Low (22) \\
\hline \multicolumn{8}{|c|}{ MRI bone cyst -- cross-sectional studies } \\
\hline Stehling 2010 [65] & $\begin{array}{l}\text { Presence of any MRI semi- } \\
\text { quantitative cyst (C) }\end{array}$ & $\begin{array}{l}\text { Presence of any } \\
\text { WORMS MRI cartilage } \\
\text { defects (C) }\end{array}$ & $\begin{array}{l}\text { Age, gender and BMI, KL } \\
\text { score, knee injury or knee } \\
\text { surgery, family history of } \\
\text { TKR and Heberden's nodes }\end{array}$ & NR & $p=0.0131$ & + & High (71) \\
\hline Torres 2006 [103] & $\begin{array}{l}\text { MRI bone cyst (WORMS) } \\
\text { TFJ and PFJ (C) }\end{array}$ & $\begin{array}{l}\text { Semi-quantitative } \\
\text { cartilage (WORMS) } \\
\text { TFJ and PFJ (C) }\end{array}$ & $\mathrm{Nil}$ & $R=0.75$ & & NC & High (68) \\
\hline Hayes 2005 [22] & $\begin{array}{l}\text { Semi-quantitative MRI bone } \\
\text { cyst }(C)\end{array}$ & $\mathrm{KL}$ grade $(\mathrm{C})$ & Nil & $p=0.02$ & NR & + & High (61) \\
\hline Link 2003 [101] & $\begin{array}{l}\text { Crude presence of MRI } \\
\text { bone cyst (C) }\end{array}$ & $\mathrm{KL}$ grade $(\mathrm{C})$ & Nil & $p<0.01$ & NR & + & Low (54) \\
\hline Crema 2010 [122] & $\begin{array}{l}\text { MRI Bone cysts } \\
\text { (WORMS) (C) }\end{array}$ & $\begin{array}{l}\text { Cartilage defect } \\
\text { (WORMS) (C) }\end{array}$ & $\mathrm{Nil}$ & NR & NR & + & Low (50) \\
\hline \multicolumn{8}{|c|}{ CT bone cyst - cross-sectional studies } \\
\hline Okazaki 2014 [40] & $\begin{array}{l}\text { Number of CT bone cysts } \\
\text { (medial femur and tibia) (C) }\end{array}$ & Knee $\mathrm{KL}$ grade $(\mathrm{C})$ & $\mathrm{Nil}$ & $p<0.05$ & Nil & $\begin{array}{l}\text { +with KL grade in } \\
\text { medial TFJ }\end{array}$ & Low (50) \\
\hline \multicolumn{8}{|c|}{ MRI subchondral bone morphometry - cohort studies } \\
\hline $\begin{array}{l}\text { Lo } 2012 \\
\text { Abstract [53] }\end{array}$ & $\begin{array}{l}\text { MRI BVF, trabecular number, } \\
\text { thickness and spacing }(C)\end{array}$ & $\begin{array}{l}\text { OARSI medial TFJ JSN } \\
\text { progression between } \\
24 \text { and } 48 \text { months }(L)\end{array}$ & Nil & $\begin{array}{l}\text { OR } 2.4,95 \% \mathrm{Cl} 1.1 \text { to } \\
5.0, p=0.02\end{array}$ & NR & $\begin{array}{l}\text { BVF, trabecular } \\
\text { number and } \\
\text { thickness are } \\
\text { positively associated } \\
\text { with JSN progression } \\
\text { but negatively } \\
\text { associated with } \\
\text { trabecular spacing. }\end{array}$ & Low (50) \\
\hline \multicolumn{8}{|c|}{ MRI subchondral bone morphometry - cross-sectional studies } \\
\hline $\begin{array}{l}\text { Driban } 2011 \\
\text { [50] Abstract }\end{array}$ & $\begin{array}{l}\text { MRI bone volume fraction, } \\
\text { trabecular number, spacing } \\
\text { \& thickness of medial tibia } \\
\text { (C) }\end{array}$ & $\begin{array}{l}\text { The presence of any } \\
\text { grade of radiographic } \\
\text { medial \& lateral JSN } \\
\text { (C) }\end{array}$ & Nil & $R=0.09$ to 1.77 & NR & $\begin{array}{l}\text { + Medial JSN } \\
\text { associated with higher } \\
\text { BVF, trabecular } \\
\text { number and thickness } \\
\text { but lower spacing }\end{array}$ & High (71) \\
\hline Driban 2011 [49] & $\begin{array}{l}\text { MRI bone volume fraction } \\
\text { (C) }\end{array}$ & Radiographic JSN (C) & $\mathrm{Nil}$ & NR & NR & $\begin{array}{l}\text { + Higher JSN score, } \\
\text { lower JSW) were } \\
\text { associated with } \\
\text { higher BVF }\end{array}$ & High (64) \\
\hline
\end{tabular}


Table 1 Knee structural associations by feature and quality grade (Continued)

\begin{tabular}{|c|c|c|c|c|c|c|c|}
\hline Lindsey 2004 [123] & $\begin{array}{l}\text { MRI bone volume fraction } \\
\text { trabecular and trabecular } \\
\text { number (TFJ) (C) }\end{array}$ & $\begin{array}{l}\text { Cartilage volume of } \\
\text { tibia or femur in } \\
\text { contralateral TFJ } \\
\text { compartment (C) }\end{array}$ & Nil & $\begin{array}{l}\text { Medial TFJ cartilage with } \\
\text { lateral TFJ BVF and } \\
\text { trabecular number. } \\
\beta=0.29 \text { to } 0.36 \\
p=0.0020 \text { to } 0.02\end{array}$ & NR & $\begin{array}{l}\text { + With contralateral } \\
\text { BVF and trabecular } \\
\text { number, but - with } \\
\text { trabecular spacing }\end{array}$ & High (64) \\
\hline Lo 2012 [54] & $\begin{array}{l}\text { MRI bone volume fraction, } \\
\text { trabecular thickness, } \\
\text { number, spacing and DXA } \\
\text { BMD of (proximal medial } \\
\text { tibia) (C) }\end{array}$ & $\begin{array}{l}\text { Radiographic medial } \\
\text { JSN grade }(C)\end{array}$ & Nil & All $p<0.0001$ & Nil & $\begin{array}{l}+(B V / T V \text {, thickness, } \\
\text { number, BMD) } \\
\text { (spacing) }\end{array}$ & High (64) \\
\hline Chiba 2012 [34] & $\begin{array}{l}\text { MRI bone volume fraction } \\
\text { and trabecular thickness of } \\
\text { the medial \& lateral femur } \\
\text { \& tibia. (C) }\end{array}$ & $\begin{array}{l}\text { Metric JSW } \\
\text { (radiographic) of the } \\
\text { medial and lateral TFJ } \\
\text { (C) }\end{array}$ & Nil & $\begin{array}{l}\text { Bone volume fraction } \\
-0.48(p<0.001) \text { trabecular } \\
\text { thickness }-0.51 \quad(p<0.001)\end{array}$ & NR & - & Low (57) \\
\hline \multicolumn{8}{|c|}{ DXA BMD - cohort studies } \\
\hline Dore 2010 [68] & $\begin{array}{l}\text { Baseline proximal tibial } \\
\text { BMD, DXA (C) }\end{array}$ & $\begin{array}{l}\text { Increase or no increase } \\
\text { in semi-quantitative MRI } \\
\text { tibial cartilage } \\
\text { defects over } 2.7 \text { years } \\
(\mathrm{L})\end{array}$ & $\begin{array}{l}\text { Age, sex, BMI, baseline } \\
\text { cartilage defects and } \\
\text { subchondral tibial } \\
\text { bone area }\end{array}$ & NR & $\begin{array}{l}\text { OR }(95 \% \mathrm{Cl}) \text { medial } \\
\text { tibia } 1.6(1.2 \text { to } 2.1) \\
p<0.01 \text { lateral tibia } \\
1.2(0.9,1.6) p=0.19\end{array}$ & $\begin{array}{l}\text { + Association only } \\
\text { observed in medial } \\
\text { tibia }\end{array}$ & Low (50) \\
\hline $\begin{array}{l}\text { Lo } 2012 \\
\text { Abstract [53] }\end{array}$ & $\begin{array}{l}\text { DXA-measured } \\
\text { medial:lateral periarticular } \\
\text { BMD (paBMD) (C) }\end{array}$ & $\begin{array}{l}\text { OARSI medial TFJ JSN } \\
\text { progression }(L)\end{array}$ & Nil & $\begin{array}{l}\text { OR } 8.4,95 \% \mathrm{Cl} 2.8 \\
\text { to } 25.0, p<0.0001\end{array}$ & nil & $\begin{array}{l}+ \text { JSN association } \\
\text { with baseline M:L } \\
\text { paBMD }\end{array}$ & Low (50) \\
\hline Bruyere 2003 [42] & $\begin{array}{l}\text { Subchondral tibial bone } \\
\text { BMD (DXA) (C) }\end{array}$ & $\begin{array}{l}\text { Minimum medial JSW } \\
\text { TFJ after one year }(L)\end{array}$ & $\begin{array}{l}\text { Age, sex, BMl, minimum } \\
\text { JSW }\end{array}$ & NR & $R=-0.43, p=0.02$ & $\begin{array}{l}\text { Negative correlation } \\
\text { i.e., lower BMD gives } \\
\text { bigger JSW or less JSN }\end{array}$ & Low (44) \\
\hline \multicolumn{8}{|c|}{ DXA BMD - cross-sectional studies } \\
\hline Dore 2009 [52] & $\begin{array}{l}\text { DXA tibial subchondral } \\
\text { BMD (C) }\end{array}$ & $\begin{array}{l}\text { Radiograph JSN grade } \\
\text { and MRI cartilage } \\
\text { defect and volume (C) }\end{array}$ & Age, sex BMl & NR & $\begin{array}{l}\text { Medial tibial BMD vs } \\
\text { JSN } R=0.11, p<0.01 \\
\text { defect } R=0.16, p<0.01, \\
\text { cartilage volume } R= \\
0.12, p=0.01\end{array}$ & $\begin{array}{l}\text { + Higher the BMD } \\
\text { the greater the JSN } \\
\text { and cartilage defects, }\end{array}$ & High (71) \\
\hline Lo 2006 [55] & $\begin{array}{l}\text { DXA medial:Iateral BMD } \\
\text { ratio at the tibial plateau (C) }\end{array}$ & $\begin{array}{l}\text { Radiographic JSN } \\
\text { grade (medial and } \\
\text { lateral TFJ) (C) }\end{array}$ & Age, sex, BMI & $p<0.0001$ & $N R$ & $\begin{array}{l}\text { + With medial JSN, - } \\
\text { with lateral JSN }\end{array}$ & High (71) \\
\hline Lo 2012 [54] & $\begin{array}{l}\text { DXA BMD (proximal medial } \\
\text { tibia) (C) }\end{array}$ & $\begin{array}{l}\text { Radiographic medial } \\
\text { JSN grade }(C)\end{array}$ & Nil & $p<0.0001$ & $N R$ & + & High (64) \\
\hline $\begin{array}{l}\text { Akamatsu } 2014 \\
\text { [31] Abstract }\end{array}$ & $\begin{array}{l}\text { BMD (DXA) (C) (medial } \\
\text { tibia and femoral condyle) }\end{array}$ & $\begin{array}{l}\text { Medial TFJ JSN } \\
\text { (radiographic) (C) }\end{array}$ & Nil & $\begin{array}{l}\text { Tibia } R=0.571, p<0.001 \\
\text { femur } R=0.550, p<0.001\end{array}$ & NR & $\begin{array}{l}\text { + Medial femoral } \\
\text { and tibial condyle } \\
\text { BMD correlated with } \\
\text { medial JSN }\end{array}$ & Low (57) \\
\hline \multicolumn{8}{|c|}{ Volumetric CT BMD - case control studies } \\
\hline Bennell 2008 [56] & & $\mathrm{KL}$ grade $(C)$ & Age, sex, BMI & NR & $p<0.05$ & $\begin{array}{l}\text { NC BMD falls in } \\
\text { posterior tibial }\end{array}$ & Low (59) \\
\hline
\end{tabular}

OARSI medial TFJ JSN

Minimum medial JSW Age, sex, BMI, minimum

nd MRl cartilage

defect $R=0.16, p<0$.

$0.12, p=0.01$

diographic media

JSN grade $(\mathrm{C})$ 
Table 1 Knee structural associations by feature and quality grade (Continued)

\section{Volumetric BMD in tibial \\ subchondral trabecular \\ bone (C)}

Knee scintigraphic subchondral bone cohort studies

Mazzuca 2004 [37] Baseline late-phase

subchondral bone

scintigraphy (adjusted for healthy diaphysis uptake) of the medial tibia and whole knee (C)

Mazzuca 2005 [38] Baseline late-phase subchondral bone scintigraphy (adjusted for healthy diaphysis uptake) of the medial tibia and whole knee (C)

Dieppe 1993 [58] Baseline late and or earlyphase subchondral bone scintigraphy signal (C)

Progression of minimum JSN of the medial TFJ from baseline to 30 months (L)

Progression of minimum JSN of the medial TFJ from baseline to 30 months $(\mathrm{L})$

Progression of JSN by $\geq 2 \mathrm{~mm}$ or knee operation incidence after 5 years $(\mathrm{L})$

Knee scintigraphic subchondral bone cross-sectional studies

Kraus 2009 [59]

Ipsilateral late-phase bone Ipsilateral OARSI scale scintigraphy, semiquantitative retention scoring of TFJ (C)

McCrae 1992 [62] Late-phase 'extended bone uptake' pattern bone scintigraphy, presence around the TFJ (C)

2D knee bone shape - cross-sectional studies

Haverkamp 2D bone shape knee.

$2011[36]$

1. Femur and tibial width
2. Elevation of lateral tibial

plateau (C) of JSN (C)

Radiographic JSN presence (C)

1. Presence of diffuse cartilage defects semiquantitative scoring (MRI). 2. Presence of ROA knee $(K L \geq 2)(C)$

\section{Age, BM} women) $r$ $r=0$ to $0.08(p<0.05)$
Baseline JSW, treatment group osteophyte OARSI score, knee alignment knee symptoms

\section{Nil}

NB (this is a population of women only) ROA models adjusted for age, BMI; cartilage defect models adjusted for $\mathrm{KL}$ only 0.22 to $0.30(p<0.05)$ NA after adjustm
for covariates

High (56)

plateau as $\mathrm{KL}$

increases but

anteriorly increase in

BMD noted

or covariates

Coefficient $0.221,95 \% \quad+$ The greater the scintigraphic bone signal the greater the JSN

$p<0.005$

NR

Low (50)

Coefficient 0.47 to 0.48 Coefficient 0.26 to $0.29+$ $(p<0.0001) \quad(p=0.0005$ to 0.001$)$

OR $47.3,95 \% \mathrm{Cl} 6.4$ to $\quad \mathrm{NR}$
OR (95\% Cl) bone width vs knee ROA $2.03(1.55$ to 2.66) $p<0.001$ bone width Presence of diffuse cartilage defects $p<0.001$
OR $(95 \% \mathrm{Cl})$ knee ROA + Wider bones and 1.94 (1.44 to 2.62) elevated tibial plateau $p<0.001$ the presence of knee. Cartilage defects were only associated with bone width Positive correlation reported between bone feature and outcome measure (+); negative correlation reported between bone feature and outcome measure (-). BMD bone mineral density, BMI body mass index, BML
bone marrow lesion, BOKS Boston osteoarthritis of the knee study, BLOKS Boston-Leeds osteoarthritis knee score, BVF bone volume fraction, C a feature or outcome described in cross-section, CT computed tomography, DXA bone marrow lesion, BOKS Boston osteoarthritis of the knee study, BLOKS Boston-Leeds osteoarthritis knee score, BVF bone volume fraction, $C$ a feature or outcome described in cross-section, CT computed tomography, DXA
dual-energy x-ray absorptiometry, GARP Genetics, osteoarthritis and progression study, JSN joint space narrowing, JSW joint space width, KL Kellgren-Lawrence, KOSS knee osteoarthritis scoring system, L a feature or outcome described longitudinally, MAK-2 mechanical factors in arthritis of the knee 2. NC no conclusion could be found for an association between bone feature and outcome measure, SWAN Michigan study of women's health across the nation, MOST multicentre osteoarthritis study, MRI magnetic resonance imaging, NA no association. NR not reported, OA osteoarthritis, OAI Osteoarthritis Initiative, OR odds ratio, RR relative risk ratio, SSR subchondral surface ratio TASOAC Tasmanian older adult cohort, TFJ tibiofemoral joint, VAS visual analogue scale, WOMAC Western Ontario and McMaster Universities arthritis index, WORMS whole-organ magnetic resonance imaging score, CRP C-reactive protein, TKR total knee replacement, OARSI Osteoarthritis Research Society International, PFJ patellofemoral joint, ROA radiographic osteoarthritis 


\section{Bone marrow lesions}

MRI (31 cohort, 15 cross-sectional, 4 case-control studies): in prospective cohorts with high- quality, well-adjusted analyses the presence and increasing size of baseline BMLs and incidence of BMLs conferred greater odds of structural progression [67, 70-73]. Similarly increasing baseline BML size increased the risk of total knee replacement (TKR) and expedited the outcome of TKR $[44,74-76]$. The association between BMLs and structural progression of OA was maintained in cohorts without clinical features of knee OA [67] and in analyses with poorer quality or statistical adjustment [25, 28, 76-90]. Only five low quality cohort analyses did not support these findings [91-95]. All cross-sectional analyses found positive correlation between BMLs and structural severity of OA [22, 29, 35, 63-65, 87, 96-103]. Three case-control analyses found similar associations [17, 104, 105]. In summary, BMLs are independently associated with structural progression of OA of the knee and incident TKR.

\section{Osteophytes}

MRI (three cohorts, eight cross-sectional studies): in one prospective cohort with high quality and well-adjusted analysis, the increasing size of osteophytes conferred greater odds of structural progression of OA [106]. In lower quality, inadequately adjusted, prospective cohorts, increasing osteophyte size increased the risk of incident TKR and structural progression of OA $[28,45]$. The increasing size and presence of osteophytes was associated with greater structural progression or severity in all included analyses [22, 26, 28, 45, 64, 65, 97, 101, 103, 106-108]. In summary, osteophytes are independently associated with knee structural progression and are associated with TKR incidence.

\section{Bone attrition}

MRI (one cohort, two cross-sectional, one case-control study): one prospective, well-adjusted, but below-averagequality cohort analysis found an association with baseline attrition severity and structural progression that became insignificant after covariate adjustment [82]. The unadjusted cross-sectional analyses and case-control analysis found similar associations with structural severity [103, 109, 110]. In summary, bone attrition is associated, but not independently so, with structural progression.

\section{Bone shape/dimension}

MRI (eight cohort, seven cross-sectional, four case-control studies): in prospective cohorts with high quality welladjusted analyses, greater baseline tibial plateau bone area conferred greater odds of structural progression of OA and incidence of TKR $[18,20,111,112]$. The same association was observed in a lower quality, prospective- cohort, well-adjusted analysis [113] and in a study of the knee in patients who predominantly had no radiographic evidence of knee OA [18]. The mismatch ratio of the femoral and tibial articulating areas was not associated with structural progression after adjustment [114], but the trochlear sulcus angle and shape was associated with cross-sectional patellofemoral structural severity demonstrated on MRI [115, 116]. All cross-sectional [23, $66,107,117]$ and case-control [118-121] analyses of tibial bone area or 3D knee bone shape found association with structural severity [23, 66, 107, 117-121]. In summary, tibial bone area is independently associated with structural progression of OA of the knee and incidence of TKR.

\section{Bone cyst}

MRI and CT (five cohort, five cross-sectional) studies: two prospective cohorts with well-adjusted but belowaverage-quality analyses of cysts reported no association with structural progression of OA before or after adjustment $[82,93]$. Two prospective cohorts with low quality unadjusted analyses of cysts found an association with structural progression of OA [77, 88]. Cross-sectional well-adjusted [65] and unadjusted [22, 40, 101, 122] cyst analyses found an association with structural severity. In summary, after covariate adjustment there is no independent association between cysts and structural progression of $\mathrm{OA}$.

\section{Trabecular bone morphometry}

MRI (one cohort, five cross-sectional studies): one prospective cohort, unadjusted, below-average-quality analysis reported increasing bone volume fraction, trabecular number and thickness and decreasing trabecular spacing were associated with structural progression [53]. The same bone changes were associated with structural severity in cross-sectional unadjusted analyses [34, 49, 50, $54,123]$. In summary, increasing bone volume fraction, trabecular number, trabecular thickness, and decreasing trabecular spacing are associated with structural progression and severity of OA of the knee.

\section{Peri-articular bone mineral density}

DXA and CT (three cohort, four cross-sectional, onecase control study): two prospective cohorts with welladjusted but below-average-quality analyses reported that increasing tibial subchondral BMD is associated with structural progression of OA $[42,68]$. In one prospective cohort with an unadjusted below-average-quality analysis, the medial-to-lateral ratio of tibial peri-articular BMD was associated with structural progression [53]. All crosssectional analyses [31, 52, 54, 55], including two that were well-adjusted [52, 55], reported increasing BMD with greater structural severity. One well-adjusted analysis using quantitative $\mathrm{CT}(\mathrm{qCT})$ reported higher and lower BMD in 
the anterior and posterior tibial plateau, respectively, in knees of patients with moderate OA relative to asymptomatic controls. In summary, increasing peri-articular radiographic BMD is associated with structural progression and severity of OA.

\section{Scintigraphy}

Scintigraphy (three cohort, two cross-sectional studies): prospective cohorts with high quality analyses found greater late-phase bone signal was associated with structural progression of $\mathrm{OA}$, with no or inadequate covariate adjustment $[37,38]$, but not after adequate covariate adjustment [37]. A prospective cohort, with below-averagequality, unadjusted analysis found greater bone signal was associated with structural progression of OA [58]. Bone signal was associated with structural severity in well-adjusted and unadjusted cross-sectional analyses [59, 62]. In summary, bone scintigraphy signal is associated, but not independently so, with structural progression of OA.

\section{D Knee bone shape}

One cross-sectional, well-adjusted analysis identified an association between greater femoral and tibial bone width and elevating tibial plateau, and greater structural severity [36]. In summary, 2D bone shape is associated with structural severity of OA.

\section{Relationship between knee bone feature and pain}

The association between bone features and pain is described in Tables 2 and 5 . In all types of study, bone features were compared with the presence, chronicity and severity of pain. In longitudinal studies, bone features were also compared with change in the presence or severity of pain (e.g., change in Western Ontario and McMaster Universities arthritis index (WOMAC) pain score). Change in the presence of pain included developing new frequent pain, [49], or the resolution of existing pain.

\section{Bone marrow lesions}

MRI ( 9 cohort, 18 cross-sectional, 5 case-control studies): in 3 prospective cohort, well-adjusted, high quality analyses the baseline or longitudinal increase in size of BMLs was associated with longitudinally increasing knee WOMAC pain severity [21, 72, 124]. This association was observed in one [28] but not two [89, 95] similar prospective-cohort, unadjusted, lower quality analyses. Baseline BML size in the lateral but not the medial tibiofemoral joint was associated with incident frequent knee pain in a prospective-cohort, well-adjusted, high quality analysis [125]. Longitudinally increasing BML size was associated with incident frequent knee pain in a similar but inadequately adjusted analysis of below average quality [126]. In cross-sectional studies the size or presence of BMLs was inconsistently associated with the presence of a heterogenous range of pain measures, irrespective of adequate covariate adjustment $[22,29,48,96,97,99$, $101,103,125,127-135]$. In summary, BMLs are independently associated with longitudinally increasing pain severity and are associated with incident frequent knee pain.

\section{Osteophytes}

MRI (one cohort, eight cross-sectional, one case-control study): one prospective cohort, unadjusted, below-averagequality analysis reported increasing baseline osteophyte size was associated with increasing WOMAC pain severity score [28]. In well-adjusted cross-sectional analyses, osteophyte size was associated with the presence [130] but not severity of pain [136]. In unadjusted cross-sectional analyses osteophytes were inconsistently associated with a heterogenous range of pain measures [22, 97, 101, 103, $127,137]$. In summary, osteophytes are associated with longitudinally increasing pain severity and the crosssectional presence of pain.

\section{Bone attrition}

MRI (no cohort, two cross-sectional, one case-control study); cross-sectional analyses found greater attrition was associated with greater pain severity, without covariate adjustment [103, 138], but not after adequate covariate adjustment [138]. An unadjusted case-control analysis found an association between attrition and prevalent pain [139]. In summary, bone attrition is associated, but not independently so, with severity of pain.

\section{Bone shape/dimension}

MRI (one cohort, one cross-sectional study): one prospective, well-adjusted, high quality analysis found the femorotibial articulating surface mismatch was associated with incident frequent knee pain [114]. One unadjusted crosssectional analysis found the irregularity of the femoral condyle surface was associated with severity of knee pain [47]. In summary, specific features of bone shape are independently associated with incident frequent knee pain and severity of pain.

\section{Bone cyst}

MRI (one cohort, five cross-sectional, two case-control studies): one prospective cohort, unadjusted, low quality analysis found no association between bone cyst size and increasing WOMAC pain score [28]. In mostly unadjusted cross-sectional $[22,101,103,130,137]$ and case control analyses [139, 140] of heterogenous cyst measures and pain measures, an association between cysts and pain was inconsistently found. In summary, bone cysts may not be associated with longitudinal severity of pain and crosssectional association with pain is uncertain. 
Table 2 Knee pain associations by feature and quality score

\begin{tabular}{|c|c|c|c|c|c|c|c|}
\hline Author & Feature (method) & Knee pain outcome & Adjustment for confounders & $\begin{array}{l}\text { Association (magnitude) } \\
\text { crude }\end{array}$ & $\begin{array}{l}\text { Association (magnitude) } \\
\text { adjusted }\end{array}$ & Association & $\begin{array}{l}\text { Quality score } \\
(\%)\end{array}$ \\
\hline \multicolumn{8}{|c|}{ MRI bone marrow lesion - cohort studies } \\
\hline \multirow[t]{2}{*}{ Foong 2014 [21] } & \multirow{2}{*}{$\begin{array}{l}\text { Change in BML size }(\mathrm{L}) \\
\text { and incident BMLs }(\mathrm{L}) \\
\text { in all three knee } \\
\text { compartments }\end{array}$} & \multirow[t]{2}{*}{$\begin{array}{l}\text { WOMAC Knee pain } \\
\text { severity at 2-year } \\
\text { and 10-year visits }(\mathrm{L})\end{array}$} & \multirow[t]{2}{*}{$\begin{array}{l}\text { Age, sex, BMl, leg strength, } \\
\text { and the presence of ROA }\end{array}$} & \multirow[t]{2}{*}{ NR } & $\begin{array}{l}\text { Incident or change in total } \\
\text { BML size } \beta=1.53(95 \% \mathrm{Cl} \\
0.37 \text { to } 2.70 \text {. }\end{array}$ & + & \multirow[t]{2}{*}{ High (67) } \\
\hline & & & & & $\begin{array}{l}\text { Medial tibial change in } \\
\text { BML size } \beta=2.96(95 \% \mathrm{Cl} \\
0.59-5.34\end{array}$ & $\begin{array}{l}\text { Incidence of BML or } \\
\text { increase in size } \\
\text { associated with increase } \\
\text { in pain in the medial tibia }\end{array}$ & \\
\hline \multirow[t]{3}{*}{ Driban 2013 [72] } & \multirow{3}{*}{$\begin{array}{l}\text { Knee baseline BML } \\
\text { volume (C), BML } \\
\text { volume change } \\
\text { (L) (TFJ) }\end{array}$} & \multirow{3}{*}{$\begin{array}{l}\text { 48-month change } \\
\text { in WOMAC pain }(L)\end{array}$} & \multirow[t]{3}{*}{ Age, sex, BMl } & \multirow[t]{3}{*}{ NR } & $\beta=0.21$ & + & \multirow[t]{3}{*}{ High (61) } \\
\hline & & & & & (standard error 0.07) & \multirow{2}{*}{$\begin{array}{l}\text { Longitudinal }(\mathrm{L}) \text { changes } \\
\text { in BML correlated with } \\
\text { (L) changes in pain severity }\end{array}$} & \\
\hline & & & & & $p=0.004$ & & \\
\hline Dore 2010 [124] & $\begin{array}{l}\text { MRI BML size }(L) \\
\text { regional or whole } \\
\text { TFJ over } 2.7 \text { years }\end{array}$ & $\begin{array}{l}\text { Change in WOMAC } \\
\text { pain }(\mathrm{L}) \text { over } \\
2.7 \text { years }\end{array}$ & $\begin{array}{l}\text { Age, sex, BMl, leg strength, } \\
\text { quality of life, and baseline } \\
\text { pain, function }\end{array}$ & $\begin{array}{l}\beta(95 \% \mathrm{Cl}) \text { Total } \\
\text { BML size change }= \\
1.06(0.10 \text { to } 2.03)\end{array}$ & $\begin{array}{l}\beta(95 \% \mathrm{Cl}) \text { total } \mathrm{BML} \\
\text { size change }=1.13(0.28 \\
\text { to } 1.98)\end{array}$ & + & High (56) \\
\hline $\begin{array}{l}\text { Kornaat } 2007 \\
\text { [173] }\end{array}$ & $\begin{array}{l}\text { Semi-quantitative } \\
\text { MRI BML change } \\
\text { over } 2 \text { years (L) TFJ }\end{array}$ & $\begin{array}{l}\text { Mean WOMAC pain } \\
\text { over } 2 \text { years }\end{array}$ & Age, sex and BMI & NR & $\beta(95 \% \mathrm{Cl})=2(-8$ to 11$)$ & NA & High (56) \\
\hline $\begin{array}{l}\text { Moisio } \\
2009 \text { [125] }\end{array}$ & $\begin{array}{l}\text { Baseline MRI semi- } \\
\text { quantitative BML } \\
\text { score (C) TFJ and PFJ }\end{array}$ & $\begin{array}{l}\text { Incident frequent } \\
\text { knee pain } 2 \text { years } \\
\text { after baseline }(\mathrm{L})\end{array}$ & $\begin{array}{l}\text { Age, sex, BMl, BML score, } \\
\% \text { denuded bone }\end{array}$ & NR & $\begin{array}{l}\text { OR }(95 \% \text { Cl) medial tibia } \\
\text { and femur } 1.41 \text { ( } 0.86 \text { to } \\
2.33) \text {, lateral tibia and } \\
\text { femur } 1.70 \text { (1.07 to } 2.69)\end{array}$ & $\begin{array}{l}\text { + Lateral TFJ BML score } \\
\text { associated with incident } \\
\text { frequent knee pain }\end{array}$ & High (56) \\
\hline $\begin{array}{l}\text { Sowers } 2011 \\
\text { [28] }\end{array}$ & $\begin{array}{l}\text { Semi-quantitative MRI } \\
\text { BML, size in TFJ (C) }\end{array}$ & $\begin{array}{l}\text { Increasing WOMAC } \\
\text { pain }(\mathrm{L})\end{array}$ & Nil & $\begin{array}{l}\text { Medial and lateral } \\
\text { TFJ BMLs both } \\
p<0.005\end{array}$ & NR & + & Low (53) \\
\hline $\begin{array}{l}\text { Zhang } 2011 \\
{[126]}\end{array}$ & $\begin{array}{l}\text { Semi-quantitative } \\
\text { change in MRI BML } \\
\text { size (L) TFJ over } \\
30 \text { months }\end{array}$ & $\begin{array}{l}\text { Incidence of } \\
\text { frequent knee } \\
\text { pain, and categorical } \\
\text { severity }(\mathrm{L}) \text { over } \\
30 \text { months }\end{array}$ & Synovitis and effusions & $\begin{array}{l}\text { OR }(95 \% \text { Cl) Severity } \\
\text { of frequent knee pain } \\
\text { OR } 3.0(1.5 \text { to } 6.0)\end{array}$ & $\begin{array}{l}\text { OR }(95 \% \mathrm{Cl}) \text { Incident } \\
\text { frequent knee pain } p \text { for } \\
\text { trend }=0.006 \text {. Severity of } \\
\text { frequent knee pain OR } \\
2.2(1.0 \text { to 4.7) } p=0.047\end{array}$ & + Ipsilateral association & Low (50) \\
\hline Wildi 2010 [95] & $\begin{array}{l}\text { 24-month change in } \\
\text { regional TFJ BML } \\
\text { score WORMS }(L)\end{array}$ & $\begin{array}{l}\text { 24-month change } \\
\text { in WOMAC pain }(\mathrm{L})\end{array}$ & Nil & $\begin{array}{l}R<0.15, p>0.067 \text { for } \\
\text { all compartments }\end{array}$ & NR & $\begin{array}{l}\text { NA, all compartments } \\
\text { had no correlation }\end{array}$ & Low (50) \\
\hline $\begin{array}{l}\text { Tanamas } 2010 \\
\text { [89] }\end{array}$ & $\begin{array}{l}\text { Baseline semi- } \\
\text { quantitative MRI BML } \\
\text { size }(C)\end{array}$ & $\begin{array}{l}\text { Annual change in } \\
\text { WOMAC pain }(\mathrm{L})\end{array}$ & Nil & NR & NR & NA & Low (50) \\
\hline \multicolumn{8}{|c|}{ MRI bone marrow lesion - cross-sectional studies } \\
\hline Zhai 2006 [135] & $\begin{array}{l}\text { Semi-quantitative MRI } \\
\text { BML (C) }\end{array}$ & WOMAC pain >1 (C) & $\begin{array}{l}\text { Age, BMl, sex, knee strength, } \\
\text { chondral defects }\end{array}$ & NR & OR 1.44, $95 \%$ Cl 1.04, 2.00 & + & High (79) \\
\hline $\begin{array}{l}\text { Sharma } 2014 \\
\text { [133] }\end{array}$ & $\begin{array}{l}\text { Semi-quantitative BML } \\
\text { score WORMS TFJ or } \\
\text { PFJ (C) }\end{array}$ & $\begin{array}{l}\text { Prevalent frequent } \\
\text { knee symptoms }(\mathrm{C})\end{array}$ & $\begin{array}{l}\text { Age, sex, body mass index } \\
\text { (BMI), previous knee injury, } \\
\text { and previous knee surgery }\end{array}$ & NR & $\begin{array}{l}\text { BMLs in any compartment } \\
\text { OR } 1.96,95 \% \mathrm{Cl} 1.38 \text { to } 2.77\end{array}$ & $\begin{array}{l}\text { + BML association with } \\
\text { prevalent knee symptoms }\end{array}$ & High (71) \\
\hline
\end{tabular}


Table 2 Knee pain associations by feature and quality score (Continued)

\begin{tabular}{|c|c|c|c|c|c|c|c|}
\hline $\begin{array}{l}\text { Kornaat } 2006 \\
{[130]}\end{array}$ & $\begin{array}{l}\text { Semi-quantitative MRI } \\
\text { BML (C) }\end{array}$ & $\begin{array}{l}\text { Chronic pain } \\
\text { presence (C) }\end{array}$ & Age, sex, and BMl & NR & $\begin{array}{l}\text { OR 1.13, } 95 \% 0.41,3.11 \\
p=0.76\end{array}$ & NA & High (71) \\
\hline Lo 2009 [131] & $\begin{array}{l}\text { Semi-quantitative MRI } \\
\text { BML (BLOKS) (C) }\end{array}$ & WOMAC pain (C) & Synovitis, effusion scores & $p$ for trend $=0.0009$ & $p$ for trend $=0.006$ & + & High (71) \\
\hline $\begin{array}{l}\text { Stefanik } 2014 \\
\text { abstract [134] }\end{array}$ & $\begin{array}{l}\text { BML (WORMS) (C) } \\
\text { (patellofemoral joint) }\end{array}$ & $\begin{array}{l}\text { Prevalent knee pain } \\
\text { (any pain in last } 30 \\
\text { days) and pain VAS (C) }\end{array}$ & $\begin{array}{l}\text { Adjusted for age, sex, BMl, } \\
\text { depressive symptoms and } \\
\text { TFJ BMLs }\end{array}$ & NR & $\begin{array}{l}\text { Isolated BML of the lateral } \\
\text { PFJ, OR ( } 95 \% \text { CI) } 1.4(0.9 \text { to } \\
\text { 2.0); medial PFJ, OR }(95 \% \mathrm{Cl}) \\
\text { 1.1 ( } 0.8 \text { to } 1.5) \text {. Isolated } \\
\text { lateral PFJ BMLs OR } 6.6(1.7 \\
\text { to } 11.5)\end{array}$ & NC & High (71) \\
\hline $\begin{array}{l}\text { Ratzlaff } 2013 \\
\text { [132] }\end{array}$ & $\begin{array}{l}\text { Total BML volume in } \\
\text { the femur or tibia (C) }\end{array}$ & $\begin{array}{l}\text { Weight-bearing } \\
\text { knee pain WOMAC } \\
\text { subscale (C) }\end{array}$ & $\begin{array}{l}\text { Age, sex, BMI, race, and } \\
\text { medial minimum joint } \\
\text { space width }\end{array}$ & NR & $\begin{array}{l}\text { Total BML volume femur } \\
p=0.003, \text { tibia } p=0.101\end{array}$ & + Femoral NA Tibial & High (71) \\
\hline \multirow[t]{2}{*}{ Ip 2011 [99] } & \multirow{2}{*}{$\begin{array}{l}\text { Semi-quantitative MRI } \\
\text { BML (C) }\end{array}$} & \multirow[t]{2}{*}{ WOMAC pain (C) } & \multirow{2}{*}{$\begin{array}{l}\text { Age, sex, BMI, OA stage, } \\
\text { joint effusion, and meniscal } \\
\text { damage }\end{array}$} & \multirow[t]{2}{*}{ NR } & Total WOMAC pain & \multirow[t]{2}{*}{ NC } & \multirow[t]{2}{*}{ High (68) } \\
\hline & & & & & $\begin{array}{l}R=0.05,95 \% \mathrm{Cl}-0.04 \text { to } \\
0.14 . \text { Stair climbing pain } \\
R=0.09(0.00 \text { to } 0.18)\end{array}$ & & \\
\hline $\begin{array}{l}\text { Torres } 2006 \\
\text { [103] }\end{array}$ & $\begin{array}{l}\text { MRI BML (WORMS) } \\
\text { TFJ and PFJ (C) }\end{array}$ & Pain VAS (C) & Age, BMl & $\begin{array}{l}\text { Coefficient 5.00, } \\
95 \% \text { Cl } 3.00 \text { to } 7.00\end{array}$ & $\begin{array}{l}\text { Coefficient } 3.72,95 \% \mathrm{Cl} \\
1.76 \text { to } 5.68\end{array}$ & + & High (68) \\
\hline Kim 2013 [129] & $\begin{array}{l}\text { Summary score and } \\
\text { severity of MRI BML } \\
\text { (WORMS) (C) }\end{array}$ & $\begin{array}{l}\text { WOMAC pain } \\
\text { severity or presence } \\
\text { of knee pain }(C)\end{array}$ & $\begin{array}{l}\text { Age, sex, BMI, radiographic } \\
\text { OA }\end{array}$ & NR & $\begin{array}{l}\text { BML summary score medial } \\
\text { TFJ OR } 2.33,95 \% \mathrm{Cl} 1.02 \text { to } \\
5.33, p<0.001\end{array}$ & $\begin{array}{l}\text { + Severity of BML is } \\
\text { proportional to WOMAC } \\
\text { in medial compartment } \\
\text { after adjustment }\end{array}$ & High (64) \\
\hline $\begin{array}{l}\text { Moisio } 2009 \\
{[125]}\end{array}$ & $\begin{array}{l}\text { Baseline MRI semi- } \\
\text { quantitative BML } \\
\text { score (C) TFJ and PFJ }\end{array}$ & $\begin{array}{l}\text { Presence of baseline } \\
\text { moderate to severe } \\
\text { knee pain (C) }\end{array}$ & $\begin{array}{l}\text { Percent denuded bone, } \\
\text { age, sex, BMl }\end{array}$ & NR & $\begin{array}{l}\text { Bone marrow lesion score, } \\
\text { OR } 0.95,95 \% \mathrm{Cl} 0.63 \text { to } \\
\text { 1.44. Not significant in all } \\
\text { compartments }\end{array}$ & $\begin{array}{l}\text { NA found on cross-sectional } \\
\text { analysis }\end{array}$ & High (64) \\
\hline $\begin{array}{l}\text { Ratzlaff } 2014 \\
\text { [48] Abstract }\end{array}$ & $\begin{array}{l}\text { Median BML volume } \\
(\text { PFJ, TFJ) (C) }\end{array}$ & $\begin{array}{l}\text { Stair-climbing knee } \\
\text { pain WOMAC }(C)\end{array}$ & Nil & $\begin{array}{l}\text { TFJ } p=0.01, \\
\text { patellofemoral } p=0.01 \\
\text { femur } p=0.02 \text {, tibia } \\
p=0.03\end{array}$ & NR & + & High (64) \\
\hline Hayes 2005 [22] & $\begin{array}{l}\text { Semi-quantitative MRI } \\
\text { BML (C) }\end{array}$ & $\begin{array}{l}\text { Chronic pain } \\
\text { presence (C) }\end{array}$ & Nil & $p=0.001$ & NR & + & High (61) \\
\hline Ai 2010 [127] & $\begin{array}{l}\text { Semi-quantitative MRI } \\
\text { BML (C) }\end{array}$ & $\begin{array}{l}\text { Pain verbal rating } \\
\text { scale (Likert) (C) }\end{array}$ & Nil & $p=0.33$ & NR & NA & Low (57) \\
\hline $\begin{array}{l}\text { Bilgici } 2010 \\
\text { [128] }\end{array}$ & MRI BML (WORMS) (C) & $\begin{array}{l}\text { WOMAC pain, pain } \\
\text { VAS (C) }\end{array}$ & Nil & $\begin{array}{l}\text { WOMAC } r=0.508 \\
p<0.01 \text { Pain VAS } \\
r=0.488, p<0.01\end{array}$ & NR & + & Low (57) \\
\hline $\begin{array}{l}\text { Sowers } 2003 \\
\text { [29] }\end{array}$ & $\begin{array}{l}\text { Semi-quantitative MRI } \\
\text { BML (C) }\end{array}$ & $\begin{array}{l}\text { Chronic pain } \\
\text { presence }(C)\end{array}$ & $\mathrm{Nil}$ & $\begin{array}{l}\text { OR } 5.0,95 \% \mathrm{Cl} 2.4 \\
\text { to } 10.5\end{array}$ & NR & + & Low (54) \\
\hline Link 2003 [101] & $\begin{array}{l}\text { Semi-quantitative MRI } \\
\text { BML (C) }\end{array}$ & WOMAC pain (C) & Nil & $p>0.05$ & NR & NA & Low (54) \\
\hline Felson 2001 [96] & & & & $p<0.001$ & OR 3.31, $95 \% \mathrm{Cl} 1.54$ to 7.41 & + & Low (54) \\
\hline
\end{tabular}


Table 2 Knee pain associations by feature and quality score (Continued)

\begin{tabular}{|c|c|c|c|c|c|c|c|}
\hline & $\begin{array}{l}\text { Semi-quantitative MRI } \\
\text { BMLs (C) }\end{array}$ & $\begin{array}{l}\text { Chronic knee pain } \\
\text { presence (C) }\end{array}$ & $\begin{array}{l}\text { Radiographic severity, age, } \\
\text { sex, and effusion score }\end{array}$ & & & & \\
\hline $\begin{array}{l}\text { Fernandez- } \\
\text { Madrid } \\
1994 \text { [97] }\end{array}$ & $\begin{array}{l}\text { Crude presence of MRI } \\
\text { BMLs (C) }\end{array}$ & $\begin{array}{l}\text { Crude pain } \\
\text { presence }(C)\end{array}$ & $\mathrm{Nil}$ & NR & NR & NA & Low (46) \\
\hline \multicolumn{8}{|c|}{ MRI bone marrow lesion - case-control studies } \\
\hline $\begin{array}{l}\text { Javaid } 2010 \\
{[140]}\end{array}$ & $\begin{array}{l}\text { Baseline semi- } \\
\text { quantitative MRI BML } \\
\text { size (WORMS) (C) TFJ } \\
\text { and PFJ }\end{array}$ & $\begin{array}{l}\text { Incident frequent } \\
\text { knee pain after } \\
15 \text { months }(L)\end{array}$ & Age, sex, race, BMl & NR & $\begin{array}{l}\text { Whole knee OR } 2.8,95 \% \mathrm{Cl} \\
1.2 \text { to } 6.5\end{array}$ & + & High (76) \\
\hline $\begin{array}{l}\text { Felson } 2007 \\
{[181]}\end{array}$ & $\begin{array}{l}\text { Semi-quantitative MRI } \\
\text { BML size increase } \\
\text { (WORMS) (L) TFJ } \\
\text { and PFJ }\end{array}$ & $\begin{array}{l}\text { Incident frequent } \\
\text { pain at } 15 \text { months } \\
\text { (L) }\end{array}$ & $\begin{array}{l}\text { Age, sex, race, BMI, } \\
\text { quadriceps strength, } \mathrm{KL} \\
\text { score, malalignment, } \\
\text { baseline BML score }\end{array}$ & $\begin{array}{l}\text { OR } 4.1,95 \% \text { Cl } 2.1 \\
\text { to } 8.1\end{array}$ & OR $3.2,95 \% \mathrm{Cl} 1.5$ to 6.8 & + & High (71) \\
\hline $\begin{array}{l}\text { Javaid } 2012 \\
\text { [139] }\end{array}$ & $\begin{array}{l}\text { Baseline Semi- } \\
\text { quantitative MRI BML, } \\
\text { (WORMS) (C) TFJ } \\
\text { and PFJ }\end{array}$ & $\begin{array}{l}\text { Presence of } \\
\text { frequent knee } \\
\text { pain }(C) \text { after } \\
2 \text { years }\end{array}$ & $\mathrm{Nil}$ & $\begin{array}{l}\text { OR } 1.70,95 \% \mathrm{Cl} \\
1.08 \text { to }\end{array}$ & NR & + & Low (59) \\
\hline Zhao 2010 [105] & $\begin{array}{l}\text { Baseline crude } \\
\text { presence of MRI BMLs } \\
\text { at (C) TFJ }\end{array}$ & $\begin{array}{l}\text { Change in WOMAC } \\
\text { Pain }(\mathrm{L})\end{array}$ & $\mathrm{Nil}$ & $p=0.60$ & $N R$ & NA & Low (56) \\
\hline Stahl 2011 [41] & $\begin{array}{l}\text { Semi-quantitative MRI } \\
\text { BML size (WORMS) } \\
\text { (L) TFJ }\end{array}$ & $\begin{array}{l}\text { Changes in WOMAC } \\
\text { score }(L)\end{array}$ & $\mathrm{Nil}$ & NR & Data not shown & NA & Low (47) \\
\hline \multicolumn{8}{|c|}{ MRI osteophyte - cohort studies } \\
\hline $\begin{array}{l}\text { Sowers } 2011 \\
\text { [28] }\end{array}$ & $\begin{array}{l}\text { Semi-quantitative MRI } \\
\text { osteophyte, size in } \\
\text { TFJ (C) }\end{array}$ & $\begin{array}{l}\text { Increasing WOMAC } \\
\text { pain }(L)\end{array}$ & Nil & $\begin{array}{l}\text { Medial and lateral } \\
\text { TFJ BMLs both } \\
p<0.001\end{array}$ & NR & + & Low (53) \\
\hline \multicolumn{8}{|c|}{ MRI osteophyte - cross-sectional studies } \\
\hline $\begin{array}{l}\text { Kornaat } 2006 \\
{[130]}\end{array}$ & $\begin{array}{l}\text { Semi-quantitative MRI } \\
\text { osteophyte (C) }\end{array}$ & $\begin{array}{l}\text { Chronic pain } \\
\text { presence }(C)\end{array}$ & Age, sex, BMl & NR & $\begin{array}{l}\text { Patellofemoral OR 2.25, } \\
95 \% \text { Cl } 1.06 \text { to } 4.77\end{array}$ & + & High (71) \\
\hline $\begin{array}{l}\text { Sengupta } \\
2006[136]\end{array}$ & $\begin{array}{l}\text { Semi-quantitative MRI } \\
\text { osteophyte (WORMS) } \\
\text { (C) }\end{array}$ & $\begin{array}{l}\text { Pain severity } \\
\text { WOMAC, chronic } \\
\text { pain (C) }\end{array}$ & Age, sex, BMl & NR & OR $0.97,95 \% \mathrm{Cl} 0.86$ to 1.10 & NA & High (71) \\
\hline $\begin{array}{l}\text { Torres } 2006 \\
\text { [103] }\end{array}$ & $\begin{array}{l}\text { MRI osteophyte, } \\
\text { (WORMS) TFJ and } \\
\text { PFJ (C) }\end{array}$ & Pain VAS (C) & Nil & $\begin{array}{l}\text { Coefficient } 1.18 \\
95 \% \mathrm{Cl} 0.63 \text { to } 1.72\end{array}$ & $\begin{array}{l}\text { Coefficient } 0.50,95 \% \mathrm{Cl} \\
0.07 \text { to } 0.94\end{array}$ & NC & High (68) \\
\hline Hayes 2005 [22] & $\begin{array}{l}\text { Semi-quantitative MRI } \\
\text { osteophyte (C) }\end{array}$ & $\begin{array}{l}\text { Chronic pain } \\
\text { presence (C) }\end{array}$ & $\mathrm{Nil}$ & $p<0.001$ & NR & + & High (61) \\
\hline Ai 2010 [127] & $\begin{array}{l}\text { Semi-quantitative MRI } \\
\text { osteophytes (C) }\end{array}$ & $\begin{array}{l}\text { Pain verbal rating } \\
\text { scale (Likert) (C) }\end{array}$ & $\mathrm{Nil}$ & $p=0.166$ & $N R$ & NA & Low (57) \\
\hline $\begin{array}{l}\text { Hayashi } 2012 \\
{[137]}\end{array}$ & $\begin{array}{l}\text { Crude presence of } \\
\text { MRI osteophytes (C) }\end{array}$ & & $\mathrm{Nil}$ & $\begin{array}{l}\text { OR } 4.2 \text { to } 6.4, p= \\
0.001-0.011\end{array}$ & $N R$ & + & Low (57) \\
\hline
\end{tabular}


Table 2 Knee pain associations by feature and quality score (Continued)

\begin{tabular}{|c|c|c|c|c|c|c|c|}
\hline & & $\begin{array}{l}\text { Presence of pain } \\
\text { on WOMAC pain } \\
\text { subscale (C) }\end{array}$ & & & & & \\
\hline Link 2003 [101] & $\begin{array}{l}\text { Semi-quantitative MRI } \\
\text { osteophytes (C) }\end{array}$ & WOMAC pain (C) & Nil & $p>0.05$ & NR & NA & Low (54) \\
\hline $\begin{array}{l}\text { Fernandez- } \\
\text { Madrid } 1994 \\
\text { [97] }\end{array}$ & $\begin{array}{l}\text { Crude presence of MRI } \\
\text { osteophytes (C) }\end{array}$ & $\begin{array}{l}\text { Crude pain } \\
\text { presence }(C)\end{array}$ & Nil & NR & NR & NA & Low (46) \\
\hline \multicolumn{8}{|c|}{ MRI osteophyte - case-control studies } \\
\hline $\begin{array}{l}\text { Javaid } 2010 \\
\text { [140] }\end{array}$ & $\begin{array}{l}\text { Baseline semi- } \\
\text { quantitative MRI } \\
\text { osteophyte, size } \\
\text { (WORMS) (C) TFJ } \\
\text { and PFJ }\end{array}$ & $\begin{array}{l}\text { Incident frequent } \\
\text { knee pain after } \\
15 \text { months }(L)\end{array}$ & Age, sex, race, BMl & NR & $\begin{array}{l}\text { Whole knee severe osteophyte } \\
\text { OR } 4.7,95 \% \mathrm{Cl} 1.3 \text { to } 18\end{array}$ & + & High (76) \\
\hline \multicolumn{8}{|c|}{ MRI bone attrition - cross-sectional studies } \\
\hline $\begin{array}{l}\text { Hernandez- } \\
\text { Molina 2008 } \\
\text { [138] }\end{array}$ & $\begin{array}{l}\text { Semi-quantitative MRI } \\
\text { bone attrition } \\
\text { (WORMS) (C) }\end{array}$ & $\begin{array}{l}\text { Pain severity and } \\
\text { nocturnal pain } \\
\text { (WOMAC) (C) }\end{array}$ & $\begin{array}{l}\text { Age, sex, BMI, BMLs, } \\
\text { effusions and KL grade }\end{array}$ & $\begin{array}{l}\text { OR }(95 \% \text { Cl) pain } \\
\text { severity OR } 1.6(1.1 \\
\text { to } 2.3) \text {, nocturnal } \\
\text { pain OR } 1.1(0.5 \\
\text { to } 2.1)\end{array}$ & $\begin{array}{l}\text { OR }(95 \% \mathrm{Cl}) \text { pain severity } \\
\text { OR } 0.9(0.6 \text { to } 1.4) \text {, nocturnal } \\
\text { pain OR } 1.0(0.5 \text { to } 2.1) \text {. }\end{array}$ & NA & High (71) \\
\hline $\begin{array}{l}\text { Torres } 2006 \\
{[103]}\end{array}$ & $\begin{array}{l}\text { MRI attrition, (WORMS) } \\
\text { TFJ and PFJ (C) }\end{array}$ & Pain VAS (C) & $\mathrm{Nil}$ & $\begin{array}{l}\text { Coefficient } 3.33 \\
95 \% \mathrm{Cl} 1.79 \text { to } 4.87\end{array}$ & $\begin{array}{l}\text { Coefficient } 1.91,95 \% \text { Cl } 0.68 \\
\text { to } 3.13\end{array}$ & + & High (68) \\
\hline \multicolumn{8}{|c|}{ MRI bone attrition - case-control studies } \\
\hline $\begin{array}{l}\text { Javaid } 2012 \\
\text { [139] }\end{array}$ & $\begin{array}{l}\text { Baseline semi- } \\
\text { quantitative MRI } \\
\text { attrition size (WORMS) } \\
\text { (C) TFJ and PFJ }\end{array}$ & $\begin{array}{l}\text { Presence of } \\
\text { frequent knee } \\
\text { pain }(C) \text { after } \\
2 \text { years }\end{array}$ & $\mathrm{Nil}$ & $\begin{array}{l}\text { OR } 2.40,95 \% \mathrm{Cl} \\
1.51 \text { to } 3.83\end{array}$ & NR & + & Low (59) \\
\hline \multicolumn{8}{|c|}{ MRI bone shape/dimension - cohort studies } \\
\hline $\begin{array}{l}\text { Everhart } 2014 \\
{[114]}\end{array}$ & $\begin{array}{l}\text { Baseline TFJ } \\
\text { subchondral surface } \\
\text { ratio of medial and } \\
\text { lateral TFJ } \\
\text { compartments (C) }\end{array}$ & $\begin{array}{l}\text { Incident frequent } \\
\text { knee pain at } \\
48 \text { months, (L) }\end{array}$ & $\begin{array}{l}\text { Sex, race, age, BMI, tobacco } \\
\text { use, activity level, knee } \\
\text { coronal alignment, baseline } \\
\text { symptoms, injury history, } \\
\text { surgery history, KL grade, } \\
\text { and JSW }\end{array}$ & NR & $\begin{array}{l}\text { Medial SSR OR } 0.48,95 \% \mathrm{Cl} \\
0.30 \text { to } 0.75, p=0.0009 . \\
\text { Lateral SSR OR } 1.27,95 \% \mathrm{Cl} \\
0.86 \text { to } 1.88, p=0.19\end{array}$ & $\begin{array}{l}\text { - larger MSSR gets less } \\
\text { incident frequent knee pain }\end{array}$ & High (61) \\
\hline \multicolumn{8}{|c|}{ MRI bone shape/dimension - cross-sectional studies } \\
\hline Ochiai 2010 [47] & $\begin{array}{l}\text { MRI irregularity of } \\
\text { femoral condyle } \\
\text { contour (C) }\end{array}$ & Knee pain VAS (C) & Nil & $\begin{array}{l}\text { Irregularity of femoral } \\
\text { condyle contour } \\
r=0.472, p=0.0021\end{array}$ & NR & + & Low (50) \\
\hline \multicolumn{8}{|c|}{ MRI bone cyst - cohort studies } \\
\hline $\begin{array}{l}\text { Sowers } 2011 \\
\text { [28] }\end{array}$ & $\begin{array}{l}\text { Semi-quantitative MRI } \\
\text { bone cyst size in } \\
\text { TFJ (C) }\end{array}$ & $\begin{array}{l}\text { Increasing WOMAC } \\
\text { pain }(\mathrm{L})\end{array}$ & $\mathrm{Nil}$ & NR & $\begin{array}{l}\text { NR analysis described as not } \\
\text { significant but data not shown }\end{array}$ & NA & Low (53) \\
\hline
\end{tabular}

MRI bone cyst - cross-sectional studies 
Table 2 Knee pain associations by feature and quality score (Continued)

\begin{tabular}{|c|c|c|c|c|c|c|c|}
\hline $\begin{array}{l}\text { Kornaat } 2006 \\
\text { [130] }\end{array}$ & $\begin{array}{l}\text { Semi-quantitative MRI } \\
\text { bone cyst(C) }\end{array}$ & $\begin{array}{l}\text { Chronic pain } \\
\text { presence }(C)\end{array}$ & Nil & NR & $\begin{array}{l}\text { Patellofemoral OR } 1.83 \text {, } \\
95 \% \mathrm{Cl}(0.80 \text { to } 4.16)\end{array}$ & NA & High (71) \\
\hline $\begin{array}{l}\text { Torres } 2006 \\
{[103]}\end{array}$ & $\begin{array}{l}\text { MRI bone cyst } \\
\text { (WORMS) TFJ and } \\
\text { PFJ (C) }\end{array}$ & Pain VAS (C) & Age, BMl & $\begin{array}{l}\text { Coefficient 2.50, } \\
95 \% \mathrm{Cl}-0.38 \text { to } 5.38\end{array}$ & $\begin{array}{l}\text { Coefficient } 0.82,95 \% \mathrm{Cl} \\
-0.50 \text { to } 2.14\end{array}$ & NA & High (68) \\
\hline Hayes 2005 [22] & $\begin{array}{l}\text { Semi-quantitative MRI } \\
\text { bone cyst }(C)\end{array}$ & $\begin{array}{l}\text { Chronic pain } \\
\text { presence }(C)\end{array}$ & Age, sex, and BMl & $p<0.001$ & NR & + & High (61) \\
\hline $\begin{array}{l}\text { Hayashi } 2012 \\
\text { [137] }\end{array}$ & $\begin{array}{l}\text { Crude presence of MRI } \\
\text { bone cysts }(C)\end{array}$ & $\begin{array}{l}\text { Presence of pain } \\
\text { on WOMAC pain } \\
\text { subscale (C) }\end{array}$ & Nil & $\begin{array}{l}\text { OR } 6.7 \text { to } 17.8, p= \\
0.004 \text { to } 0.03\end{array}$ & NR & + & Low (57) \\
\hline Link 2003 [101] & $\begin{array}{l}\text { Crude presence of MRI } \\
\text { bone cyst (C) }\end{array}$ & WOMAC pain $(C)$ & Nil & $p>0.05$ & NR & NA & Low (54) \\
\hline \multicolumn{8}{|c|}{ MRI bone cyst - case control studies } \\
\hline $\begin{array}{l}\text { Javaid } 2010 \\
\text { [140] }\end{array}$ & $\begin{array}{l}\text { Baseline semi- } \\
\text { quantitative MRI bone } \\
\text { cyst size (WORMS) (C) } \\
\text { TFJ and PFJ }\end{array}$ & $\begin{array}{l}\text { Incident frequent } \\
\text { knee pain after } \\
15 \text { months }(\mathrm{L})\end{array}$ & Nil & NR & $\mathrm{NR} p>0.1$ & NA & High (76) \\
\hline $\begin{array}{l}\text { Javaid } 2012 \\
\text { [139] }\end{array}$ & $\begin{array}{l}\text { Baseline semi- } \\
\text { quantitative MRI bone } \\
\text { cyst size (WORMS) (C) } \\
\text { TFJ and PFJ }\end{array}$ & $\begin{array}{l}\text { Presence of } \\
\text { frequent knee } \\
\text { pain }(C) \text { after } \\
2 \text { years }\end{array}$ & Nil & $\begin{array}{l}\text { OR } 1.61,95 \% \mathrm{Cl} \\
1.03 \text { to } 2.52\end{array}$ & NR & + & Low (59) \\
\hline \multicolumn{8}{|c|}{$\mathrm{qCT}$ bone mineral density - cross-sectional studies } \\
\hline $\begin{array}{l}\text { Burnett } 2012 \\
\text { [57] }\end{array}$ & $\begin{array}{l}\text { BMD of patellar lateral } \\
\text { facet }(\mathrm{qCT})(\mathrm{C})\end{array}$ & $\begin{array}{l}\text { WOMAC - knee } \\
\text { pain at rest (C) }\end{array}$ & Nil & $\begin{array}{l}\text { Total lateral patella } \\
\text { facet } p=0.04 \text {, inferior } \\
\text { lateral facet } p=0.005\end{array}$ & NR & & Low (57) \\
\hline \multicolumn{8}{|c|}{ 2D Knee bone shape - cross-sectional studies } \\
\hline $\begin{array}{l}\text { Haverkamp } 2011 \\
\text { [36] }\end{array}$ & $\begin{array}{l}\text { 2D Bone shape knee, } \\
\text { 1. femur and tibial } \\
\text { width, 2. elevation of } \\
\text { lateral tibial plateau (C) }\end{array}$ & Pain severity VAS (C) & $\begin{array}{l}\text { Models adjusted for Age, } \\
\text { BMI }\end{array}$ & NR & $\begin{array}{l}\text { Bone width } p=0.167 \text {, } \\
\text { lateral tibia plateau elevation } \\
p=0.002\end{array}$ & $\begin{array}{l}\text { + Lateral tibial plateau } \\
\text { associated with pain } \\
\text { severity, NA bone width } \\
\text { with pain severity }\end{array}$ & Low (46) \\
\hline
\end{tabular}

Positive correlation reported between bone feature and outcome measure (+); negative correlation reported between bone feature and outcome measure (-). BMI body mass index, BML bone marrow lesion, $C$ a feature or
outcome described in cross-section, knee pain on most days for at least the last month (chronic pain) confidence interval (CC), KL Kellgren-Lawrence, $L$ a feature or outcome described longitudinally, NA no association, $N C$ no outcome described in cross-section, knee pain on most days for at least the last month (chronic pain) confidence interval (CC), KL Kellgren-Lawrence, $L$ a feature or outcome described longitudinally, $N A$ no association, $N C$ no
conclusion could be found for an association between bone feature and outcome measure, NR not reported, OA osteoarthritis, OAI Osteoarthritis Initiative, OR odds ratio, PFJ patellofemoral joint, ROA radiographic osteoarthritis, SSR subchondral surface ratio VAS visual analogue scale, WOMAC Western Ontario and McMaster Universities arthritis index, qCT quantitative computed tomography 


\section{$2 D$ Knee bone shape}

One inadequately adjusted cross-sectional analysis found an association between the elevation of the lateral tibial plateau and severity of pain [36]. In summary, 2D lateral tibial bone shape is associated with cross-sectional severity of pain.

\section{Relationship between hand bone feature and structural progression}

The association between bone features and structural progression is described in Tables 3 and 5 .

\section{Bone marrow lesions}

MRI (one case series, two cross-sectional studies): one well-adjusted, high quality analysis of a prospective OA case series, found that increasing BML number and size in the interphalangeal joints at baseline conferred greater odds of structural progression of OA [141]. Two adjusted cross-sectional analyses found increasing BML number and size scores were associated with increasing severity of structural progression [142, 143]. In summary, BMLs are independently associated with structural progression of hand OA.

\section{Osteophyte attrition and cysts}

One cross-sectional, adjusted analysis found greater MRI attrition or MRI osteophyte number and size was associated with greater structural severity [142]. However, greater presence of cysts observed on MRI was not associated with greater structural severity of OA [142]. In summary, osteophytes and attrition, but not cysts, are associated with structural severity of hand OA.

\section{Relationship between hand bone feature and pain}

The association between bone features and pain is described in Tables 4 and 5.

\section{Bone marrow lesions}

MRI (one case series, one cross-sectional study): one welladjusted, high quality analysis of a prospective OA case series, found that BML number and size at baseline was not associated with longitudinal change in hand pain [144]. One adjusted cross-sectional analysis found no association of BMLs with severity of pain [145]. In summary, BMLs are not independently associated with longitudinal or cross-sectional severity of pain.

\section{Osteophyte attrition and cysts}

One cross-sectional, adjusted analysis found no association between bone features, osteophytes, attrition or cysts observed on MRI, and pain severity [145]. In summary, osteophytes, attrition and cysts are not associated with severity of hand pain.

\section{Scintigraphy}

Scintigraphy (one cross-sectional study): one cross-sectional unadjusted analysis found no significant association between bone signal in the hands and severity of pain. In summary, bone scintigraphy signal is not associated with severity of pain in hand OA.

\section{Relationship between hip bone feature and structural progression}

The association between bone features, and structural progression and joint replacement is described in Tables 3 and 5 .

\section{Bone marrow lesions}

MRI (two cross-sectional studies): one well-adjusted [69] and one unadjusted [46] cross-sectional analysis both found that BMLs were associated with greater structural severity. In summary, BMLs are associated with structural severity of hip OA.

\section{Trabecular bone morphometry}

One unadjusted cross-sectional analysis found greater MRI bone volume fraction, trabecular thickening, trabecular number and lower trabecular spacing were associated with greater structural severity of OA [33]. In summary, bone volume fraction, trabecular thickening, number and spacing are associated with structural severity in hip OA.

\section{Peri-articular bone mineral density}

DXA (two cross-sectional studies): one well-adjusted [30] and one adjusted [32] cross-sectional analysis found greater BMD was associated with greater structural severity. In summary, BMD is associated with structural severity of hip OA.

\section{$2 D$ and $3 D$ hip bone shape}

Hip bone shape (three cohort, two cross-sectional, three case-control studies): in two prospective cohort, welladjusted, high quality analyses increasing asphericity of the femoral head (measured as an elevated alpha angle, or in shape modes 11 and 15) was associated with total hip replacement (THR) [146] or with structural progression and THR [147] respectively. In one prospective cohort, well-adjusted, high quality analysis, acetabular undercoverage of the femoral head (a low centre-edge angle) was associated with structural progression or THR [148]. In one well-adjusted cross-sectional analysis, 2D asphericity deformity of the femoral head (cam-type deformity) was associated with structural severity [149]. In one well-adjusted cross-sectional analysis of MRI-determined femoral head asphericity in asymptomatic young men, there was a significantly lower cartilage thickness in those with than those without any detectable asphericity. This became insignificant after covariate adjustment [27]. Case-control analyses 
Table 3 Hand, hip and ankle structural associations by feature and quality grade

\begin{tabular}{|c|c|c|c|c|c|c|c|}
\hline Author & Feature (method) & $\begin{array}{l}\text { Structural severity or } \\
\text { progression outcome }\end{array}$ & Adjustment for confounders & $\begin{array}{l}\text { Association (magnitude) } \\
\text { crude }\end{array}$ & $\begin{array}{l}\text { Association (magnitude) } \\
\text { adjusted }\end{array}$ & Association & $\begin{array}{l}\text { Quality } \\
\text { (score \%) }\end{array}$ \\
\hline \multicolumn{8}{|c|}{ Hand MRI bone marrow lesion case series } \\
\hline \multirow{2}{*}{$\begin{array}{l}\text { Haugen } 2014 \\
\text { [141] }\end{array}$} & \multirow{2}{*}{$\begin{array}{l}\text { BMLs - semi-quantitative } \\
\text { at } 2^{\text {nd }} \text { to } 5^{\text {th }} I \mathrm{PJs}(\mathrm{C})\end{array}$} & \multirow{2}{*}{$\begin{array}{l}\text { Progression of hand } \\
\mathrm{ROA} \text { (JSN, } \mathrm{KL} \text { grade } \\
\text { or new erosion) (L) }\end{array}$} & \multirow[t]{2}{*}{ Age, sex, BMl, } & \multirow{2}{*}{$\begin{array}{l}\text { OR } 2.73,95 \% \text { Cl } 1.29 \\
\text { to } 5.78\end{array}$} & \multirow[t]{2}{*}{ NR } & + & \multirow[t]{2}{*}{ High (61) } \\
\hline & & & & & & $\begin{array}{l}\text { Bigger the BML, the } \\
\text { more the JSN }\end{array}$ & \\
\hline \multicolumn{8}{|c|}{ Hand MRI bone marrow lesion cross-sectional studies } \\
\hline \multirow{2}{*}{$\begin{array}{l}\text { Haugen } 2012 \\
\text { Abstract [143] } 299\end{array}$} & \multirow{2}{*}{$\begin{array}{l}\text { BML (Oslo MRI hand } \\
\text { score) (C) IPJs }\end{array}$} & \multirow{2}{*}{$\begin{array}{l}\text { Radiographic JSN } \\
\text { grade IPJ (OARSI } \\
\text { atlas) (C) }\end{array}$} & \multirow[t]{2}{*}{ Age, sex, } & \multirow{2}{*}{$\begin{array}{l}\text { OR } 10.0,95 \% \mathrm{Cl} 4.2 \\
\text { to } 23\end{array}$} & \multirow[t]{2}{*}{ OR $4.4,95 \% \mathrm{Cl} 2.2$ to 9.0} & + & \multirow[t]{2}{*}{ Low (43) } \\
\hline & & & & & & $\begin{array}{l}\text { BML score association } \\
\text { with more JSN }\end{array}$ & \\
\hline \multirow{3}{*}{$\begin{array}{l}\text { Haugen } 2012 \\
\text { [142] }\end{array}$} & \multirow{3}{*}{$\begin{array}{l}\text { BML (Oslo MRI hand score) } \\
\text { (C) IPJs }\end{array}$} & \multirow{3}{*}{$\begin{array}{l}\text { Hand KL grade of } \\
\text { IPJs (C) }\end{array}$} & \multirow[t]{3}{*}{ Age, sex } & \multirow[t]{3}{*}{ NR } & OR $(95 \% \mathrm{Cl})$ & + & \multirow[t]{3}{*}{ High (64) } \\
\hline & & & & & BMLs 11 (5.5 to 21) & & \\
\hline & & & & & $p<0.001$ & & \\
\hline
\end{tabular}

Hand MRI osteophyte cross-sectional studies

\begin{tabular}{|c|c|c|}
\hline $\begin{array}{l}\text { Haugen } 2012 \\
\text { [142] }\end{array}$ & $\begin{array}{l}\text { Osteophyte (Oslo MRI hand } \\
\text { score) (C) IPJs }\end{array}$ & $\begin{array}{l}\text { Hand } K L \text { grade of } \\
\text { IPJs }(C)\end{array}$ \\
\hline
\end{tabular}

NR

OR $(95 \% \mathrm{Cl})$

osteophytes

415 (189 to 908$)$

$p<0.001$

Hand MRI attrition cross-sectional studies

Attrition (Oslo MRI hand

[142]

score) (C) IPJs

Hand KL grade of

IPJS (C)

Age, sex

Hand KL grade of Age, sex

IPJS (C)

Semi-quantitative cartilage lesions (C)

$\begin{array}{lll}\begin{array}{l}\text { Neumann 2007 } \\ \text { [46] }\end{array} & \text { Semi-quantitative BMLs (C) } & \begin{array}{l}\text { Semi-quantitative } \\ \text { cartilage lesions (C) }\end{array} \\ \text { Dawson 2013 } & \text { Femoral head BMLs (MRI) (C) } & \begin{array}{l}\text { 1. Presence of hip } \\ \text { OA. 2. Femoral } \\ \text { Abstract [69] }\end{array} \\ & & \text { head cartilage } \\ & \text { volume (MRI) (C) }\end{array}$

$\mathrm{Nil}$

Age, sex, BMI
Hand MRI bone cyst cross-sectional studies

$\begin{array}{lll}\text { Haugen } 2012 & \text { Cyst (Oslo MRI hand score) } & \begin{array}{l}\text { Hand KL grade of } \\ \text { [142] }\end{array} \\ \text { (C) IPJs } & \text { Age, sex (C) }\end{array}$

volume (MRI) (C)
$N R$

OR

(37 to 204)

$p<0.001$

OR $(95 \% \mathrm{Cl})$

cysts 2.0 (0.6 to 6.3)

$p=0.26$

$R=0.44, p \leq 0.001 \quad$ NR

NR

OA hip presence

OR $(95 \% \mathrm{Cl})$

5.32 (1.78 to 15.9$)$

$p=0.003$

cartilage volume

regression coefficient

$(95 \% \mathrm{Cl})$
High (64)

High (64)

High (64)

Nil

+ Correlation between BML and cartilage lesions

$+$

BMLs associated with diagnosis of hip OA

BMLs inversely associated with cartilage volume
Low (43)

Low (14) 
Table 3 Hand, hip and ankle structural associations by feature and quality grade (Continued)

$-245.7 \mathrm{~mm}^{3}$

$(-456$ to -36$) p=0.02$

Hip CT bone morphometry cross-sectional studies

Chiba 2011 [33] Acetabular and femoral

Hip joint space

head subchondral trabecular volume (CT) (C)

morphometry: bone volume

fraction, trabecular thickness,

number, separation (CT) (C)

Hip DXA BMD cross-sectional studies

Chaganti $2010 \quad$ Femoral neck BMD (C) DXA [30]

Hip ROA Modified Croft score (categorical 0-4) (C)

Age, BMI, height, activity evel, race, 6-m walk pace,

NR

Femoral head Bone volume fraction $r=-0.691, p<0.001$

strength, inability to do
chair stands, and clinic site,

Antoniades 2000 DXA BMD of the femoral neck of left (nondominant) hip with $\mathrm{ROA}(\mathrm{C})$

Radiographic OA (Croft score) (C)

BMI, lifetime physical activity, menopausal status, use of oestrogen, and smoking

OR $1.63,95 \% \mathrm{Cl} 1.06$ to 2.50 )

Age, sex, BMI, shape modes

THR at or within

5 years $(\mathrm{L})$

Agricola 2013 Baseline 2D femoral and

acetabular shape modes

(segmented by statistical

shape modelling) (C)

Agricola 2013 Baseline alpha angle (2D

[147] femur shape) dichotomous abnormal $>60^{\circ}$, normal $\leq 60^{\circ}(\mathrm{C})$

Agricola 2013 Baseline 2D centre edge

[148] Baseline $2 \mathrm{D}$ centre edge $25^{\circ}<$ normal $<40^{\circ}$, undercoverage $<25^{\circ}$ overcoverage $>40^{\circ}(\mathrm{C})$

Incident ROA hip ( $K L>1$ ), incident end-stage ROA hip (KL >2 or THR) at or within 5 years (L)

Incidence within 5 years of: 1 . ROA hip $(K L>1)$, 2. endstage $\mathrm{OA}(\mathrm{KL}>2$

or THR)

Age, sex, BMI, KL grade

associated with THR

OR 1.71 to 2.01,

$p \leq 0.001$

OR (95\% Cl) Incident

ROA hip $6.82(3.55$

to 13.10$) p<0.0001$

Age, sex, BMI, KL grade

OR $(95 \% \mathrm{Cl})$

overcoverage 0.52

(0.19 to 1.43$) p=0.2$

undercoverage 3.64

(1.91 to 6.99) $p=0.00$

2D and 3D hip bone shape cross-sectional studies

Gosvig 2010 [149] Categorical hip 2D deformity: 1. normal, 2.'pistol grip',

3) deep acetabular socket (C) (JSW $\leq 2 \mathrm{~mm}$ ) (C)

Joint space narrowing

is associated with

increased bone volume

fraction, trabecular

thickening. trabecular

number and spacing

decrease

$p<0.0001$

High (64)

Higher BMD for higher grade of OA of hip

OR $1.80,95 \%$ Cl 1.05 to 3.12

+ Association between BMD and hip ROA grade in the index hip

Higher OA grade means higher BMD

3 modes were associated

head asphericity is

$p \leq 0.00$

associated with THR

OR (95 \% Cl) incident

ROA hip 2.42 (1.15 to

5.06) $p=0.02$, incident

severe ROA or THR 3.67

(1.68 to 8.01$) p<0.0001$

+ Elevated alpha

angle is associated

with incident end-

stage OA hip

OR $(95 \% \mathrm{Cl})$ overcoverage Overcoverage is

0.34 (0.13 to 0.87$) p=\quad$ protective against

0.025 , undercoverage OA incidence $(-)$.

5.45 (2.40 to 12.34 )

Undercoverage is

associated with

greater odds of OA

incidence and end-

stage OA (+) 
Table 3 Hand, hip and ankle structural associations by feature and quality grade (Continued)

Reichenbach The presence or absence of $2011[27]$ any $3 \mathrm{D}$ semi-quantitative MRI-defined cam-deformity (C) Combined femora and acetabular cartilage thickness were young men)

2D hip bone shape case control studies

Doherty 2008 [43] Non-spherical femoral head 2D shape assessment: 1. appearance of 'pistol grip deformity' (C), 2 maximum femoral head diamet femora head diameter ratio neck diameter $(\mathrm{C})$

Barr 2012 [150]２D Shape measures of centre-ed shape) (C)

Presence of radiographic hip OA (JSW $\leq 2.5$ $\mathrm{mm})(\mathrm{C})$

\section{THR vs no} 5 years $(\mathrm{L})$

Nicholls 2011 [39] CAM deformity; mean modified triangular index height, alpha angle. 2D lateral center edge angle, (C)

Total hip replacement $(\mathrm{L})$ type 3 hand (index finger shorter than ring finger), hand nodes, and centeredge angle function, duration of pain Age, BMI (NB all participants acetabular dysplasia; mean radiographic

Age, sex, BMI, BMD, physical activity, history of hip injury,

\section{Age, gender, BMl KL grade, $\quad$ OR $(95 \%$ Cl) mode 2 OR (95\% Cl) Mode 2} $p>0.05$

$0.17(0.04$

$\mathrm{p}<0.05$

$\mathrm{OR}$ ( $p$ value) triangular index 1.131 (0.021) Alpha angle 1.056 $(<0.0005)$. Centre edge
angle $0.906(0.004)$ Alpha angle 1.057 $(<0.0005)$. Centre- edge angle $0.887(0.002)$

NR

Age, gender, BMI

Tibiotalar ROA KL Kraus 2013 [60] scintigraphy, retention presence in tibiotalar grade and JSN (C)

0.62 to $0.75(p<0.01)$

OR (95\% CI) pistol grip deformity 6.95 (4.64 to neck ratio $12.08(8.05$

Knupp 2009 [24] Late phase bone scintigraphy, Tibiotalar ankle joint Nil

\section{OR ( $p$ value) Triangular} index $1.291(0.011)$. Ipsilateral late phase bone

$.4(2.0$ to 2.9$) p<0.001$

Adjusted mean cartilage

thickness difference with

CAM deformity $-0.19 \mathrm{~mm}$

NC

High (64) erence with CAM semi-quantitative retention JSN. (modified scoring of tibiotalar joint (C) Takakura score) (C)
$\mathrm{NB}$, this model association is inverse and correlates with Association of hip replacement with CAM impingement and acetabular by these results dysplasia indicated

Positive correlation was reported between bone feature and outcome measure (+); negative correlation reported between bone feature and outcome measure (-).BMD bone mineral density, $B M L$ bone marrow lesion, C a feature or outcome described in cross-section, CT computed tomography, DXA dual-energy x-ray absorptiometry, HOAMS Hip osteoarthritis MRI scoring system, IPJ interphalangeal joint, JSN joint space narrowing, JSW joint space width, KL Kellgren-Lawrence, $L$ a feature or outcome described longitudinally, NA no association, $N C$ no conclusion could be found for an association between bone feature and outcome measure, MRI magnetic resonance imaging, PFJ patellofemoral joint, ROA radiographic osteoarthritis, OA osteoarthritis, OARSI Osteoarthritis Research Society International, OR odds ratio, RR relative risk, TFJ tibiofemoral joint, THR total hip replacement, TKR total knee replacement, VAS visual analogue scale, WOMAC Western Ontario and McMaster Universities arthritis index, WORMS whole-organ magnetic resonance imaging score 
Table 4 Hand and hip pain associations by feature and quality score

\begin{tabular}{|c|c|c|c|c|c|c|c|}
\hline Author & Feature (method) & Pain outcome & $\begin{array}{l}\text { Adjustment for } \\
\text { confounders }\end{array}$ & $\begin{array}{l}\text { Association (magnitude) } \\
\text { crude }\end{array}$ & $\begin{array}{l}\text { Association (magnitude) } \\
\text { adjusted }\end{array}$ & Association & Quality score (\%) \\
\hline \multicolumn{8}{|c|}{ Hand MRI bone marrow lesion case series } \\
\hline $\begin{array}{l}\text { Haugen } 2014 \text { Abstract } \\
\text { [144] }\end{array}$ & $\begin{array}{l}\text { Sum scores (0-48) for BMLs } \\
\text { (Oslo hand OA MRI score) (C) }\end{array}$ & AUSCAN pain scale (L) & $\begin{array}{l}\text { Age, sex, BMl, } \\
\text { follow-up time }\end{array}$ & NR & $\begin{array}{l}\beta=-0.26,95 \% \mathrm{Cl} \\
-0.55 \text { to } 0.03\end{array}$ & NA & High (61) \\
\hline \multicolumn{8}{|c|}{ Hand MRI bone marrow lesion cross-sectional studies } \\
\hline Haugen 2012 [145] & $\begin{array}{l}\text { BML (Oslo MRI hand score) } \\
\text { (C) IPJs sum scores }\end{array}$ & AUSCAN pain scale $(C)$ & Age, sex & NR & $\begin{array}{l}\mathrm{OR}(95 \% \mathrm{Cl}) 0.96 \\
(0.82 \text { to } 1.12)\end{array}$ & NA & High (64) \\
\hline \multicolumn{8}{|c|}{ Hand MRI osteophyte cross-sectional studies } \\
\hline Haugen 2012 [145] & $\begin{array}{l}\text { Osteophyte (Oslo MRI hand } \\
\text { score) (C) IPJs sum scores }\end{array}$ & AUSCAN pain scale (C) & Age, sex & NR & $\begin{array}{l}\text { OR }(95 \% C l) 1.04 \\
(0.98 \text { to } 1.10)\end{array}$ & NA & High (64) \\
\hline \multicolumn{8}{|c|}{ Hand MRI attrition cross-sectional studies } \\
\hline Haugen 2012 [145] & $\begin{array}{l}\text { Attrition (Oslo MRI hand } \\
\text { score) (C) IPJs sum scores }\end{array}$ & AUSCAN pain scale $(C)$ & Age, sex & NR & $\begin{array}{l}\text { OR }(95 \% C l) 1.15 \\
(0.98 \text { to } 1.34)\end{array}$ & NA & High (64) \\
\hline \multicolumn{8}{|c|}{ Hand MRI subchondral cyst cross-sectional studies } \\
\hline Haugen 2012 [145] & $\begin{array}{l}\text { Cyst (Oslo MRI hand score) } \\
\text { (C) IPJs sum scores }\end{array}$ & AUSCAN pain scale $(C)$ & Age, sex & NR & $\begin{array}{l}\text { OR }(95 \% \text { Cl) } 0.93 \\
(0.56 \text { to } 1.55)\end{array}$ & NA & High (64) \\
\hline \multicolumn{8}{|c|}{ Hand scintigraphy subchondral bone cross-sectional studies } \\
\hline Macfarlane 1993 [61] & $\begin{array}{l}\text { Late phase isotope bone scan } \\
\text { small joints of the hand }(C)\end{array}$ & Hand pain VAS (C) & Nil & $\begin{array}{l}\text { Correlation coefficient } \\
0.06, p=0.304\end{array}$ & NR & NA & Low (57) \\
\hline \multicolumn{8}{|c|}{ Hip MRI bone marrow lesion cross-sectional studies } \\
\hline Kumar 2013 [151] & $\begin{array}{l}\text { Total hip semi-quantitative } \\
\text { BML score (C) }\end{array}$ & $\begin{array}{l}\text { Self-reported hip pain } \\
\text { HOOS score (C) }\end{array}$ & Nil & NR & $\begin{array}{l}\text { p correlation }-0.29 \\
(p<0.01)\end{array}$ & $\begin{array}{l}\text { A higher BML score means } \\
\text { a lower or worse HOOS } \\
\text { pain score }\end{array}$ & High (71) \\
\hline $\begin{array}{l}\text { Maksymowych } 2014 \\
\text { [152] }\end{array}$ & $\begin{array}{l}\text { Semi-quantitative BML HIP } \\
\text { (HOAMS) (C) }\end{array}$ & Baseline WOMAC pain (C) & $\mathrm{Nil}$ & $p<0.001$ & NR & + & High (64) \\
\hline \multicolumn{8}{|c|}{ Hip MRI subchondral cyst cross-sectional studies } \\
\hline Kumar 2013 [151] & $\begin{array}{l}\text { Total hip semi-quantitative } \\
\text { subchondral cyst score (C) }\end{array}$ & $\begin{array}{l}\text { Self-reported hip pain } \\
\text { HOOS score (C) }\end{array}$ & Nil & NR & $\begin{array}{l}\text { p correlation }-0.37 \\
(p<0.001)\end{array}$ & $\begin{array}{l}\text { A higher cyst score means a } \\
\text { lower or worse HOOS pain } \\
\text { score }\end{array}$ & High (71) \\
\hline
\end{tabular}

Positive correlation reported between bone feature and outcome measure (+); negative correlation reported between bone feature and outcome measure (-). AUSCAN Australian/Canadian Osteoarthritis hand index, $B M L$ bone marrow lesion, $C$ a feature or outcome described in cross-section, chronic pain knee pain on most days for at least the last month, HOAMS Hip osteoarthritis MRI scoring system, HOOS Hip dysfunction and osteoarthritis outcome score, IPJ interphalangeal joint, $L$ a feature or outcome described longitudinally, NA no association, NR not recorded, OA osteoarthritis, OR odds ratio, VAS visual analogue scale 
Table 5 The summary subchondral bone associations with joint replacement, structural progression and pain in peripheral OA

\begin{tabular}{|c|c|c|c|c|c|c|c|}
\hline \multirow{2}{*}{$\begin{array}{l}\text { Subchondral bone feature of } \\
\text { OA }\end{array}$} & \multicolumn{7}{|c|}{ Pain and structural associations } \\
\hline & Knee structure & Knee pain & Hand structure & Hand pain & Hip structure & Hip pain & Ankle structure \\
\hline \multirow[t]{2}{*}{ MRI bone marrow lesions } & Progression (i) & LPS (i) & Progression (i) & No LPS (W) & Severity (w) & Severity (n) & \\
\hline & TKR (i) & $\operatorname{IFP}(n)$ & & No severity (n) & & & \\
\hline \multirow[t]{2}{*}{ MRI osteophytes } & Progression (i) & LPS (n) & Severity (n) & No severity (n) & & & \\
\hline & $\operatorname{TKR}(n)$ & & & & & & \\
\hline MRI bone attrition & No progression (0) & No severity (0) & Severity (n) & No severity (n) & & & \\
\hline \multirow[t]{2}{*}{ MRI bone shape or dimensions } & Progression (i) & IFP (i) & & & No severity (0) & & \\
\hline & TKR (i) & Severity (n) & & & & & \\
\hline \multirow[t]{2}{*}{ MRI bone cyst } & No progression & No LPS (n) & No severity (n) & No severity (n) & & Severity (n) & \\
\hline & ?severity & & & & & & \\
\hline $\begin{array}{l}\text { MRI or CT trabecular } \\
\text { morphometry }\end{array}$ & Progression (n) & & & & Severity (n) & & \\
\hline DXA or CT Peri-articular BMD & Progression (n) & & & & Severity (w) & & \\
\hline \multirow[t]{2}{*}{ 2D Bone shape } & Severity (w) & Severity (n) & & & Progression (i) & & \\
\hline & & & & & THR (i) & & \\
\hline Scintigraphy & No Progression (0) & & & No severity (n) & & & Severity (w) \\
\hline
\end{tabular}

CT computed tomography, dual-energy DXA x-ray absorptiometry, (i) independent association, IFP incident frequent pain, ( $n$ ) association with no or inadequate covariate adjustment, TKR total knee replacement, THR total hip replacement, LPS mean change in longitudinal pain severity, ( $w$ ) well-adjusted association, (0) association insignificant after covariate adjustment

identified the same associations as the cohort analyses $[39,43,150]$. In summary, asphericity of the femoral head and acetabular undercoverage of the femoral head are independently associated with structural progression and THR.

\section{Relationship between hip bone feature and pain}

The association between bone features and pain is described in Tables 4 and 5.

\section{Bone marrow lesions}

MRI (two cross-sectional studies): two cross-sectional, unadjusted analyses found that increasing semi-quantitative BML scores were associated with greater severity of pain [151, 152]. In summary, BMLs are associated with severity of pain in hip OA.

\section{Bone cyst}

One cross-sectional, unadjusted analysis found that increasing semi-quantitative cyst scores on MRI were associated with greater severity of pain [151]. In summary: cysts are associated with severity of pain in hip OA.

\section{Relationship between ankle bone features and structural progression}

The association between bone features and structure is described in Table 3 and 5.

\section{Scintigraphy}

Scintigraphy (two cross-sectional studies): one well-adjusted [59] and one unadjusted [62] cross-sectional analysis found the presence or semi-quantitative scoring of late-phase bone signal in the tibiotalar joint was associated with greater structural severity. In summary, bone scintigraphy signal is associated with ankle structural severity.

\section{Discussion}

This systematic review is the first to have incorporated quality scoring alongside statistical adjustment in the comprehensive examination of the relationship of subchondral bone pathology with both structural progression of OA and pain for all non-conventional types of radiographic imaging of peripheral joints with OA. This systematic review has concluded that there are independent associations between imaging-assessed bone pathology and structural progression and pain in the knee, hand, and hip.

Subchondral bone pathology may lead to cartilage degeneration by altering the biomechanical force distribution across joint cartilage, or disruption of the osteochondral junction and release of soluble biomediators influencing the cartilage [153, 154]. In OA the homeostatic process of subchondral bone remodeling fails, leading to increased bone turnover, volume and change in stiffness and shockabsorbing capacity [155-157]. BMLs histologically represent increased bone turnover [158]. Cartilage overlying 
altered bone has been observed to have greater damage than healthy bone in knees from human cadavers [159]. That study, and an excluded study [160], concur with the independent association between BMLs, and structural progression of $\mathrm{OA}$ in knees and hands and total knee replacement, as concluded by this analysis. Although randomised control trials were not excluded from this review, several such trials were excluded on the basis of failure to formally quantify any correlation between BMLs and structural progression outcomes. These include the strontium [161], intensive weight-loss therapy [162] and glucosamine [163] trials, and some of these describe a concordant reduction in BML size and cartilage volume loss.

Osteophytes represent subchondral bone hypertrophy typical of OA. They represent endochondral and direct bone formation and create a circumferential increase in bone area around each knee cartilage plate, particularly on the medial side in OA [118], which concurs with the independent association between osteophytes demonstrated on MRI and structural progression as observed in this analysis.

In terms of bone morphology, knee $\mathrm{OA}$ is associated with shallow trochlear patellar grooves in multiple epiphyseal dysplasia [164]. These findings concur with the findings of Stefanik and Kalichman, and colleagues in studies of knee OA in this review [115, 116, 165]. Anteriorcruciate ligament $(\mathrm{ACL})$ rupture represents a risk factor for developing knee OA. In cases of ACL tear in previously normal knees of young healthy adults, the 3D shape of the femur, tibia and patella expands more rapidly than in controls without radiographic evidence of knee OA in the subsequent 5 years [166]. The 3D shape of the same knee bones has also been associated with the outcome of joint replacement [167]. This highlights the importance of bone shape and concurs with our conclusion that 3D knee shape and 2D hip shape are independently associated with structural progression of $\mathrm{OA}$ and total joint replacement.

We found that bone attrition and cysts were associated with structural progression or severity, but not after covariate adjustment, which included other OA subchondral bone features. This suggests these bone features are an epiphenomenon of the pathogenic process of structural progression rather than a primary cause. This hypothesis is supported by bone cysts and attrition frequently occurring synchronously with BMLs $[88,138]$ and incident bone attrition has been strongly associated with the presence of BMLs within the same compartment [168].

Increasing bone volume fraction, trabecular number and thickness, but decreasing trabecular spacing on CT and MRI studies were associated with structural progression. These specific associations concur with numerous histological analyses of peripheral joint OA [169-171].
Subchondral bone, particularly BMLs, have been found to be associated with pain in knee, hip and hand OA. However, some analyses, in which pain was measured using heterogenous pain outcomes, report an absence of longitudinal or cross-sectional association with BMLs [101, 172, 173]. Furthermore, previous systematic reviews have concluded moderate association at the most between BMLs and knee pain $[7,14]$. With the benefit of incorporating more well-adjusted analyses in this systematic review, we have highlighted that BMLs are independently longitudinally associated with change in severity of pain, but are only associated with incident frequent knee pain. In analyses excluded from the current review, incident knee BMLs predicted incident knee pain in healthy community-based adults at risk of OA [174]. Concurrent trends in reduction of pain and BML size were observed in the zoledronic acid trial [175] and the intensive diet and exercise for arthritis trial [176]. These were not included because they did not make a formal comparison of pain and BMLs. The mechanism by which BMLs may cause pain is unknown but may include subchondral microfractures, angina from a decreased blood supply causing ischaemia, and raised intraosseous pressure [177-179].

The independent association between a mismatch of the femoral and tibial articulating surface areas and incident frequent knee symptoms indicates that bone shape may predict not only the incidence of radiographic knee OA [120], but also symptomatic OA.

In terms of limitations, stratifying observational studies by quality may artificially create relatively high quality studies from a collection of generally low quality studies. However the distribution and summary statistics of quality scores indicate a suitably broad range of quality, particularly in the influential cohort studies with a mean of $54 \%$ and range of $22-83 \%$. The decision to exclude articles reporting analysis of association that included fewer than 20 patients with OA may seem arbitrary. However, several papers report associations with the presence or absence of pain or structural progression based upon small numbers of patients. Our threshold decision reflects the absence of specific guidelines on how to exclude such papers, with inherent risk of imprecision, in the context of heterogenous populations and statistical analyses. Had these papers been included there would have been no change in any of the conclusions in Table 5 (data not shown). The use of joint replacement as an outcome measure has a number of limitations including the effect of patient willingness, variation in orthopaedic opinion, availability of health services and health insurance, and therefore may be influenced depending upon the country and context in which the study is performed.

Publication bias could not be assessed with a funnel plot as there were insufficient results for odds and relative risk ratios. The heterogenous nature of the measures 
of bone features and structural or pain outcomes precluded a meta-analysis or calculation of an effect size. This was because there were insufficient analyses describing the same association between the same bone features and outcome measure pair.

\section{Conclusions}

In conclusion subchondral bone plays an integral role in the pathogenesis of OA. BMLs, osteophytes identified on MRI and tibial bone area are independently associated with structural progression of knee OA. BMLs and tibial bone area are independently associated with TKR. BMLs are independently associated with structural progression of hand $\mathrm{OA}$ and 2D hip bone shape is associated with progression of structural hip OA and THR. BMLs are independently associated with longitudinal change in severity of pain and femorotibial articulating area mismatch is independently associated with incident frequent knee pain. These bone features may be used in the future for targeting treatment, stratifying patients into those most in need of OA modification and measuring treatment response.

\section{Additional file}

Additional file 1: Supplementary material: supplementary methods and results. (DOC $1659 \mathrm{~kb}$ )

\section{Abbreviations}

2D: two-dimensional; 3D: three-dimensional; ACL: anterior cruciate ligament; BLOKS: Boston-Leeds osteoarthritis knee score; BMD: bone mineral density; BMl: body mass index; BML: bone marrow lesion; BOKS: Boston osteoarthritis of the knee study; BVF: bone volume fraction; Cam: a resemblance to a camshaft; CRP: C-reactive protein; CT: computed tomography; DMOAD: disease-modifying osteoarthritis drug; DXA: dual-energy x-ray absorptiometry; EMBASE: Excerpta Medica database; GARP: Genetics, osteoarthritis and progression study; HOAMS: Hip osteoarthritis MRI scoring system; IPJ: interphalangeal joint; JSN: joint space narrowing; JSW: joint space width; KL: Kellgren-Lawrence; KOSS: knee osteoarthritis scoring system; MOST: multicentre osteoarthritis study; MRI: magnetic resonance imaging; NA: no association; NC: no conclusion; OA: osteoarthritis; OAl: Osteoarthritis Initiative; OARSI: Osteoarthritis Research Society International; OR: odds ratio; PET: positron emission tomography; PFJ: patellofemoral joint;

PRISMA: Preferred reporting items for systematic reviews and meta-analyses; qCT: quantitative computed tomography; ROA: radiographic osteoarthritis; RR: relative risk ratio; SSR: subchondral surface ratio; THR: total hip replacement; TFJ: tibiofemoral joint; TKR: total knee replacement; VAS: visual analogue scale; WOMAC: Western Ontario and McMaster Universities arthritis index; WORMS: whole-organ magnetic resonance imaging score.

\section{Competing interests}

Dr Bowes is an employee and shareholder of Imorphics Ltd. Professor Conaghan, Sarah Kingsbury, Andrew Barr, Devan Hopkinson and Thomas Mark Campbell have nothing to disclose.

\section{Authors' contributions}

$A B$ carried out conception and design, eligibility assessment, extraction of data, quality assessment, along with drafting and revising of the manuscript content. DH carried out design, eligibility assessment and extraction of data. TC carried out conception and design, quality assessment and revising manuscript for content. MB carried out conception and design along with revising the manuscript for content. SK carried out conception and design, eligibility assessment and revising the manuscript for content. PC carried out conception and design, quality assessment and revising the manuscript for content. All authors read and approved the final manuscript version for publication.

\section{Acknowledgements}

This study has been part funded by Arthritis Research UK (Grant numbers 20154 and 20083) and the National Institute for Health Research (NIHR) through the Leeds Musculoskeletal Biomedical Research Unit. This article/paper/report presents independent research funded by the NIHR. The views expressed are those of the authors and not necessarily those of the National Health Service, the NIHR or the Department of Health.

\section{Author details}

${ }^{1}$ Leeds Institute of Rheumatic and Musculoskeletal Medicine, University of Leeds and NIHR Leeds Musculoskeletal Biomedical Research Unit, Chapeltown Rd, Leeds LS7 4SA, UK. '² Department of Medicine, University of Ottawa, Ottawa, Canada. ${ }^{3}$ Imorphics Ltd, Kilburn House, Manchester, UK.

Received: 22 December 2014 Accepted: 3 August 2015

Published online: 25 August 2015

\section{References}

1. Felson DT, Naimark A, Anderson J, Kazis L, Castelli W, Meenan RF. The prevalence of knee osteoarthritis in the elderly. The Framingham Osteoarthritis Study. Arthritis Rheum. 1987:30:914-8.

2. Bijlsma JW, Berenbaum F, Lafeber FP. Osteoarthritis: an update with relevance for clinical practice. Lancet. 2011;377:2115-26.

3. EMA. European Medicines Agency. Guideline on clinical investigation of medicinal products used in the treatment of osteoarthritis. 2010. http:// www.ema.europa.eu/docs/en_GB/document_library/Scientific_guideline/ 2009/09/WC500003440.pdf

4. FDA. Food and Drug Administration. Guidance for industry: Clinical development programs for drugs, devices, and biological products intended for the treatment of osteoarthritis (OA). 1999. FDA document 07/1999.

5. Guermazi A, Niu J, Hayashi D, Roemer FW, Englund M, Neogi T, et al. Prevalence of abnormalities in knees detected by MRI in adults without knee osteoarthritis: population based observational study (Framingham Osteoarthritis Study). BMJ. 2012;345.

6. Barr AJ, Dube B, Hensor EM, Kingsbury SR, Peat G, Bowes MA, et al. The relationship between clinical characteristics, radiographic osteoarthritis and $3 \mathrm{D}$ bone area: data from the osteoarthritis initiative. Osteoarthritis Cartilage. 2014;22:1703-9.

7. Hunter DJ, Zhang W, Conaghan PG, Hirko K, Menashe L, Li L, et al. Systematic review of the concurrent and predictive validity of MRI biomarkers in OA. Osteoarthritis Cartilage. 2011;19:557-88.

8. Conaghan PG, Felson D, Gold G, Lohmander S, Totterman S, Altman R. MRI and non-cartilaginous structures in knee osteoarthritis. Osteoarthritis Cartilage. 2006;14:A87-94.

9. Betancourt MC, Linden JC, Rivadeneira F, Rozendaal RM, Zeinstra SM Weinans $\mathrm{H}$, et al. Dual energy $\mathrm{x}$-ray absorptiometry analysis contributes to the prediction of hip osteoarthritis progression. Arthritis Res Ther. 2009;11:R162.

10. Boegard T. Radiography and bone scintigraphy in osteoarthritis of the knee-comparison with MR imaging. Acta Radiol Suppl. 1998;418:7-37.

11. Boegard T, Rudling O, Dahlstrom J, Dirksen H, Petersson IF, Jonsson K. Bone scintigraphy in chronic knee pain: comparison with magnetic resonance imaging. Ann Rheum Dis. 1999;58:20-6.

12. Temmerman OP, Raijmakers PG, Kloet R, Teule GJ, Heyligers IC, Lammertsma AA. In vivo measurements of blood flow and bone metabolism in osteoarthritis. Rheumatol Int. 2013;33:959-63.

13. Johnston JD, Masri BA, Wilson DR. Computed tomography topographic mapping of subchondral density (CT-TOMASD) in osteoarthritic and normal knees: methodological development and preliminary findings. Osteoarthritis Cartilage. 2009;17:1319-26.

14. Yusuf E, Kortekaas MC, Watt I, Huizinga TW, Kloppenburg M. Do knee abnormalities visualised on MRI explain knee pain in knee osteoarthritis? A systematic review. Ann Rheum Dis. 2011;70:60-7.

15. Stroup DF, Berlin JA, Morton SC, Olkin I, Williamson GD, Rennie D, et al. Meta-analysis of observational studies in epidemiology: a proposal for 
reporting. Meta-analysis Of Observational Studies in Epidemiology (MOOSE) group. JAMA. 2000;283:2008-12.

16. van Tulder M, Furlan A, Bombardier C, Bouter L. Updated method guidelines for systematic reviews in the cochrane collaboration back review group. Spine (Phila Pa 1976). 2003;28:1290-9.

17. Aitken D, Khan HI, Ding C, Blizzard L, Pelletier JP, Martel-Pelletier J, et al. Structural predictors of ten year knee cartilage volume loss. Arthritis Rheum. 2013;65:S97-8.

18. Ding C, Cicuttini F, Scott F, Cooley H, Boon C, Jones G. Natural history of knee cartilage defects and factors affecting change. Arch Intern Med. 2006;166:651-8.

19. Ding C, Garnero P, Cicuttini F, Scott F, Cooley H, Jones G. Knee cartilage defects: association with early radiographic osteoarthritis, decreased cartilage volume, increased joint surface area and type II collagen breakdown. Osteoarthritis Cartilage. 2005;13:198-205.

20. Ding C, Martel-Pelletier J, Pelletier JP, Abram F, Raynauld JP, Cicuttini F, et al. Two-year prospective longitudinal study exploring the factors associated with change in femoral cartilage volume in a cohort largely without knee radiographic osteoarthritis. Osteoarthritis Cartilage. 2008;16:443-9.

21. Foong YC, Khan HI, Blizzard L, Ding C, Cicuttini F, Jones G, et al. The clinical significance, natural history and predictors of bone marrow lesion change over eight years. Arthritis Res Ther. 2014;16:R149.

22. Hayes CW, Jamadar DA, Welch GW, Jannausch ML, Lachance LL, Capul DC, et al. Osteoarthritis of the knee: comparison of MR imaging findings with radiographic severity measurements and pain in middle-aged women. Radiology. 2005;237:998-1007.

23. Jones G, Ding C, Scott F, Glisson M, Cicuttini F. Early radiographic osteoarthritis is associated with substantial changes in cartilage volume and tibial bone surface area in both males and females. Osteoarthritis Cartilage. 2004;12:169-74.

24. Knupp M, Pagenstert Gl, Barg A, Bolliger L, Easley ME, Hintermann B. SPECT-CT compared with conventional imaging modalities for the assessment of the varus and valgus malaligned hindfoot. J Orthop Res. 2009;27:1461-6.

25. Koster IM, Oei EHG, Hensen JHJ, Boks SS, Koes BW, Vroegindeweij D, et al. Predictive factors for new onset or progression of knee osteoarthritis one year after trauma: MRI follow-up in general practice. Eur Radiol. 2011;21:1509-16.

26. McCauley TR, Kornaat PR, Jee WH. Central osteophytes in the knee: prevalence and association with cartilage defects on MR imaging. AJR Am J Roentgenol. 2001;176:359-64.

27. Reichenbach S, Leunig M, Werlen S, Nuesch E, Pfirrmann CW, Bonel H, et al. Association between cam-type deformities and magnetic resonance imaging-detected structural hip damage: a cross-sectional study in young men. Arthritis Rheum. 2011;63:4023-30.

28. Sowers M, Karvonen-Gutierrez CA, Jacobson JA, Jiang Y, Yosef M. Associations of anatomical measures from MRI with radiographically defined knee osteoarthritis score, pain, and physical functioning. J Bone Joint Surg Am. 2011;93:241-51.

29. Sowers MF, Hayes C, Jamadar D, Capul D, Lachance L, Jannausch M, et al. Magnetic resonance-detected subchondral bone marrow and cartilage defect characteristics associated with pain and $x$-ray-defined knee osteoarthritis. Osteoarthritis Cartilage. 2003;11:387-93.

30. Chaganti RK, Parimi N, Lang T, Orwoll E, Stefanick ML, Nevitt M, et al. Bone mineral density and prevalent osteoarthritis of the hip in older men for the Osteoporotic Fractures in Men (MrOS) Study Group. Osteoporos Int. 2010;21:1307-16

31. Akamatsu Y, Kobayashi H, Kusayama Y, Kumagai K, Mitsugi N, Saito T. Does subchondral sclerosis protect progression of joint space narrowing in patients with varus knee osteoarthritis? Osteoarthritis Cartilage. 2014;22.

32. Antoniades L, MacGregor AJ, Matson M, Spector TD. A cotwin control study of the relationship between hip osteoarthritis and bone mineral density. Arthritis Rheum. 2000;43:1450-5.

33. Chiba K, Ito $M$, Osaki $M$, Uetani $M$, Shindo $H$. In vivo structural analysis of subchondral trabecular bone in osteoarthritis of the hip using multidetector row CT. Osteoarthritis Cartilage. 2011;19:180-5.

34. Chiba K, Uetani M, Kido Y, Ito M, Okazaki N, Taguchi K, et al. Osteoporotic changes of subchondral trabecular bone in osteoarthritis of the knee: a 3-T MRI study. Osteoporosis Int. 2012;23:589-97.

35. Guymer E, Baranyay F, Wluka AE, Hanna F, Bell RJ, Davis SR, et al. A study of the prevalence and associations of subchondral bone marrow lesions in the knees of healthy, middle-aged women. Osteoarthritis Cartilage. 2007;15:1437-42
36. Haverkamp DJ, Schiphof D, Bierma-Zeinstra SM, Weinans H, Waarsing JH. Variation in joint shape of osteoarthritic knees. Arthritis Rheum. 2011;63:3401-7.

37. Mazzuca A, Brandt D, Schauwecker S, Buckwalter A, Katz P, Meyer M, et al. Bone scintigraphy is not a better predictor of progression of knee osteoarthritis than Kellgren and Lawrence grade. J Rheumatol. 2004;31:329-32.

38. Mazzuca A, Brandt D, Schauwecker S, Katz P, Meyer M, Lane A, et al. Severity of joint pain and Kellgren-Lawrence grade at baseline are better predictors of joint space narrowing than bone scintigraphy in obese women with knee osteoarthritis. J Rheumatol. 2005;32:1540-6.

39. Nicholls AS, Kiran A, Pollard TC, Hart DJ, Arden CP, Spector T, et al. The association between hip morphology parameters and nineteen-year risk of end-stage osteoarthritis of the hip: a nested case-control study. Arthritis Rheum. 2011;63:3392-400.

40. Okazaki N, Chiba K, Kidera K, Yonekura A, Osaki M. Relationship between subchondral bone cysts, the severity of knee osteoarthritis, and alignments of lower extremities. Osteoarthritis Cartilage. 2014;22:S370-1.

41. Stahl R, Jain SK, Lutz J, Wyman BT, Le MPM, Vignon E, et al. Osteoarthritis of the knee at 3.0 T: comparison of a quantitative and a semi-quantitative score for the assessment of the extent of cartilage lesion and bone marrow edema pattern in a 24-month longitudinal study. Skeletal Radiology. 2011;40:1315-27.

42. Bruyere O, Dardenne C, Lejeune E, Zegels B, Pahaut A, Richy F, et al Subchondral tibial bone mineral density predicts future joint space narrowing at the medial femoro-tibial compartment in patients with knee osteoarthritis. Bone. 2003;32:541-5.

43. Doherty M, Courtney P, Doherty S, Jenkins W, Maciewicz RA, Muir K, et al. Nonspherical femoral head shape (pistol grip deformity), neck shaft angle, and risk of hip osteoarthritis: a case-control study. Arthritis Rheum. 2008;58:3172-82

44. Hochberg MC, Yip A, Favors K, Sorkin J, Martel-Pelletier J, Pelletier JP. Features assessed on magnetic resonance images improve prediction of total knee arthroplasty in subjects with symptomatic radiographic knee osteoarthritis: Data from the Osteoarthritis Initiative. Osteoarthritis Cartilage. 2014;22:S175.

45. Liu L, Kaneko H, Sadatsuki R, Hada S, Yusup A, Kinoshita M, et al. MRIdetected osteophyte is a predictor for receiving total knee arthroplasty in patients with end-stage knee osteoarthritis. Osteoarthritis Cartilage. 2014;22:S470-1.

46. Neumann G, Mendicuti AD, Zou KH, Minas T, Coblyn J, Winalski CS, et al. Prevalence of labral tears and cartilage loss in patients with mechanical symptoms of the hip: evaluation using MR arthrography. Osteoarthritis Cartilage. 2007;15:909-17.

47. Ochiai N, Sasho T, Tahara M, Watanabe A, Matsuki K, Yamaguchi S, et al. Objective assessments of medial osteoarthritic knee severity by MRI: new computer software to evaluate femoral condyle contours. Int Orthop. 2010;34:811-7.

48. Ratzlaff C, Russell R, Duryea J. Quantitatively-measured bone marrow lesions in the patellofemoral joint: Distribution and association with pain. Osteoarthritis Cartilage. 2014;22:S247-8.

49. Driban JB, Price LL, Tassinari AM, Lo GH, McAlindon TE. Peri-articular apparent bone volume fraction is associated with numerous patient characteristics in knees with osteoarthritis: Data from the osteoarthritis initiative. Arthritis Rheum. 2011;63.

50. Driban JB, Price LL, Tassinari AM, Lo GH, Schneider E, McAlindon TE. Trabecular morphology is associated with numerous patient characteristics in knees with osteoarthritis: Data from the osteoarthritis initiative (OAI). Osteoarthritis Cartilage. 2011;19:s169.

51. Altman RD, Hochberg M, Murphy Jr WA, Wolfe F, Lequesne M. Atlas of individual radiographic features in osteoarthritis. Osteoarthritis Cartilage. 1995;3:3-70.

52. Dore D, Quinn S, Ding C, Winzenberg T, Jones G. Correlates of subchondral BMD: a cross-sectional study. J Bone Miner Res. 2009;24:2007-15.

53. Lo GH, Schneider E, Price L, Driban J, Tassinari A, Nevitt M, et al. Periarticular bone density and trabecular morphology predict knee OA structural progression. Osteoarthritis Cartilage. 2012;20:S76.

54. Lo GH, Tassinari AM, Driban JB, Price LL, Schneider E, Majumdar S, et al. Cross-sectional DXA and MR measures of tibial periarticular bone associate with radiographic knee osteoarthritis severity. Osteoarthritis Cartilage. 2012;20:686-93.

55. Lo GH, Zhang Y, McLennan C, Niu J, Kiel DP, McLean RR, et al. The ratio of medial to lateral tibial plateau bone mineral density and compartment-specific tibiofemoral osteoarthritis. Osteoarthritis Cartilage. 2006;14:984-90. 
56. Bennell KL, Creaby MW, Wrigley TV, Hunter DJ. Tibial subchondral trabecular volumetric bone density in medial knee joint osteoarthritis using peripheral quantitative computed tomography technology. Arthritis Rheum. 2008:58:2776-85.

57. Burnett WK, McLennan SA, Wheaton CE, Talmo D, Hunter C, Wilson DJ, et al. Patella Bone density is lower in knee osteoarthritis patients experiencing pain at rest. Osteoarthritis Cartilage. 2012;20:S200-1.

58. Dieppe P, Cushnaghan J, Young P, Kirwan J. Prediction of the progression of joint space narrowing in osteoarthritis of the knee by bone scintigraphy. Ann Rheum Dis. 1993;52:557.

59. Kraus VB, McDaniel G, Worrell TW, Feng S, Vail TP, Varju G, et al. Association of bone scintigraphic abnormalities with knee malalignment and pain. Ann Rheum Dis. 2009;68:1673.

60. Kraus VB, Worrell TW, Renner JB, Coleman RE, Pieper CF. High prevalence of contralateral ankle abnormalities in association with knee osteoarthritis and malalignment. Osteoarthritis and cartilage/OARS, Osteoarthritis Research Society. 2013;21:1693.

61. Macfarlane DG, Buckland-Wright JC, Lynch J, Fogelman L. A study of the early and late 99technetium scintigraphic images and their relationship to symptoms in osteoarthritis of the hands. Br J Rheumatol. 1993:32:977-81.

62. McCrae F, Shouls J, Dieppe P, Watt I. Scintigraphic assessment of osteoarthritis of the knee joint. Ann Rheum Dis. 1992;51:938.

63. Baranyay FJ, Wang Y, Wluka AE, English DR, Giles GG, Sullivan RO, et al. Association of bone marrow lesions with knee structures and risk factors for bone marrow lesions in the knees of clinically healthy, community-based adults. Semin Arthritis Rheum. 2007;37:112-8.

64. Meredith DS, Losina E, Neumann G, Yoshioka H, Lang PK, Katz JN. Empirical evaluation of the inter-relationship of articular elements involved in the pathoanatomy of knee osteoarthritis using magnetic resonance imaging BMC Musculoskelet Disord. 2009;10.

65. Stehling C, Lane NE, Nevitt MC, Lynch J, McCulloch CE, Link TM. Subjects with higher physical activity levels have more severe focal knee lesions diagnosed with $3 \mathrm{~T} \mathrm{MRI}$ : Analysis of a non-symptomatic cohort of the osteoarthritis initiative. Osteoarthritis Cartilage. 2010;18:776-86.

66. Wang $Y$, Wluka $A E$, Cicuttini FM. The determinants of change in tibial plateau bone area in osteoarthritic knees: a cohort study. Arthritis Res Ther. 2005;7:R687-93.

67. Davies-Tuck ML, Wluka AE, Forbes A, Wang Y, English DR, Giles GG, et al. Development of bone marrow lesions is associated with adverse effects on knee cartilage while resolution is associated with improvement-a potential target for prevention of knee osteoarthritis: a longitudinal study. Arthritis Res Ther. 2010;12:R10.

68. Dore D, Quinn S, Ding C, Winzenberg T, Cicuttini F, Jones G. Subchondral bone and cartilage damage: a prospective study in older adults. Arthritis Rheum. 2010;62:1967-73.

69. Dawson L, Bennell K, Wluka A, Wang Y, Cicuttini F. Hip bone marrow lesions in asymptomatic and osteoarthritic adults: Prevalence, risk factors and significance. Osteoarthritis and cartilage / OARS, Osteoarthritis Research. Society. 2013;21:S241.

70. Felson DT, McLaughlin S, Goggins J, LaValley MP, Gale ME, Totterman S, et al. Bone marrow edema and its relation to progression of knee osteoarthritis. Ann Intern Med. 2003;139:330-6.

71. Crema MD, Cibere J, Sayre EC, Roemer FW, Wong H, Thorne A, et al. The relationship between subchondral sclerosis detected with MRI and cartilage loss in a cohort of subjects with knee pain: the knee osteoarthritis progression (KOAP) study. Osteoarthritis Cartilage. 2014;22:540-6.

72. Driban JB, Price LL, Lo GH, Pang J, Hunter DJ, Miller E, et al. Evaluation of bone marrow lesion volume as a knee osteoarthritis biomarker longitudinal relationships with pain and structural changes: Data from the Osteoarthritis Initiative. Arthritis Res Ther. 2013;15.

73. Guermazi A, Eckstein F, Hayashi D, Roemer FW, Wirth W, Yang T, et al. Cartilage damage, bone marrow lesions and meniscal lesions predict quantitatively measured loss of cartilage over 30 months: The most study. Osteoarthritis Cartilage. 2014;22:S356.

74. Raynauld JP, Martel-Pelletier J, Dorais M, Haraoui B, Choquette D, Abram F, et al. Total knee replacement as a knee osteoarthritis outcome: predictors derived from a 4-year long-term observation following a randomized clinical trial using chondroitin sulfate. Cartilage. 2013:4:219-26.

75. Raynauld JP, Martel-Pelletier J, Haraoui B, Choquette D, Dorais M, Wildi LM, et al. Risk factors predictive of joint replacement in a 2-year multicentre clinical trial in knee osteoarthritis using MRI: Results from over 6 years of observation. Ann Rheum Dis. 2011;70:1382-8.

76. Dore D, Martens A, Quinn S, Ding C, Winzenberg T, Zhai G, et al. Bone marrow lesions predict site-specific cartilage defect development and volume loss: a prospective study in older adults. Arthritis Res Ther. 2010;12:R222.

77. Carrino JA, Blum J, Parellada JA, Schweitzer ME, Morrison WB. MRI of bone marrow edema-like signal in the pathogenesis of subchondral cysts. Osteoarthritis Cartilage. 2006;14:1081-5.

78. Crema MD, Felson DT, Roemer FW, Wang K, Marra MD, Nevitt MC, et al. Prevalent cartilage damage and cartilage loss over time are associated with incident bone marrow lesions in the tibiofemoral compartments: the MOST study. Osteoarthritis and cartilage / OARS, Osteoarthritis Research Society. 2013;21:306-13.

79. Driban JB, Lo GH, Lee JY, Ward RJ, Miller E, Pang J, et al. Quantitative bone marrow lesion size in osteoarthritic knees correlates with cartilage damage and predicts longitudinal cartilage loss. BMC Musculoskelet Disord. 2011;12:217

80. Driban JB, Pang J, Miller E, Destenaves G, Lo GH, Ward RJ, et al. Quantitative bone marrow lesion changes relate to cartilage parameter changes. Osteoarthritis Cartilage. 2012;20:S217-8.

81. Hernandez-Molina G, Guermazi A, Niu J, Gale D, Goggins J, Amin S, et al, Central bone marrow lesions in symptomatic knee osteoarthritis and their relationship to anterior cruciate ligament tears and cartilage loss. Arthritis Rheum. 2008:58:130-6.

82. Kothari A, Guermazi A, Chmiel JS, Dunlop D, Song J, Almagor O, et al. Within-subregion relationship between bone marrow lesions and subsequent cartilage loss in knee osteoarthritis. Arthritis Care Res. 2010;62:198-203.

83. Parsons $\mathrm{CEMH}$, Bruye`re O, Belissa P, Genant HK, Guermazi A, Roemer F, et al. Impact of bone marrow lesion on the progression of knee osteoarthritis in the sekoia study. Rheumatology. 2014;53:i130.

84. Pelletier JP, Raynauld JP, Berthiaume MJ, Abram F, Choquette D, Haraoui B, et al. Risk factors associated with the loss of cartilage volume on weight-bearing areas in knee osteoarthritis patients assessed by quantitative magnetic resonance imaging: a longitudinal study. Arthritis Res Ther. 2007;9.

85. Raynauld JP, Martel-Pelletier J, Berthiaume MJ, Abram F, Choquette D, Haraoui B, et al. Correlation between bone lesion changes and cartilage volume loss in patients with osteoarthritis of the knee as assessed by quantitative magnetic resonance imaging over a 24-month period. Ann Rheum Dis. 2008;67:683-8.

86. Roemer FW, Kwoh CK, Hannon MJ, Green SM, Jakicic JM, Boudreau R, et al. Risk factors for magnetic resonance imaging-detected patellofemoral and tibiofemoral cartilage loss during a six-month period: the joints on glucosamine study. Arthritis Rheum. 2012;64:1888-98.

87. Scher C, Craig J, Nelson F. Bone marrow edema in the knee in osteoarthrosis and association with total knee arthroplasty within a three-year follow-up. Skeletal Radiol. 2008;37:609-17.

88. Tanamas SK, Wluka AE, Pelletier JP, Martel-Pelletier J, Abram F, Wang Y, et al. The association between subchondral bone cysts and tibial cartilage volume and risk of joint replacement in people with knee osteoarthritis: a longitudinal study. Arthritis Res Ther. 2010;12:R58.

89. Tanamas SK, Wluka AE, Pelletier JP, Pelletier JM, Abram F, Berry PA, et al. Bone marrow lesions in people with knee osteoarthritis predict progression of disease and joint replacement: a longitudinal study. Rheumatology. 2010:49:2413-9.

90. Roemer FW, Guermazi A, Javaid MK, Lynch JA, Niu J, Zhang Y, et al. Change in MRI-detected subchondral bone marrow lesions is associated with cartilage loss: the MOST Study. A longitudinal multicentre study of knee osteoarthritis. Ann Rheum Dis. 2009;68:1461-5.

91. Hunter DJ, Zhang Y, Niu J, Goggins J, Amin S, LaValley MP, et al. Increase in bone marrow lesions associated with cartilage loss: a longitudinal magnetic resonance imaging study of knee osteoarthritis. Arthritis Rheum. 2006;54:1529-35.

92. Kubota M, Ishijima M, Kurosawa H, Liu L, Ikeda H, Osawa A, et al. A longitudinal study of the relationship between the status of bone marrow abnormalities and progression of knee osteoarthritis. J Orthop Sci. 2010;15:641-6

93. Madan-Sharma R, Kloppenburg M, Kornaat PR, Botha-Scheepers SA, Le MPH, Bloem $J$, et al. Do MRI features at baseline predict radiographic joint space narrowing in the medial compartment of the osteoarthritic knee 2 years later? Skeletal Radiol. 2008:37:805-11.

94. Roemer FW, Zhang Y, Niu J, Lynch JA, Crema MD, Marra MD, et al. Tibiofemoral joint osteoarthritis: Risk factors for MR-depicted fast cartilage 
loss over a 30-month period in the multicenter osteoarthritis study. Radiology. 2009;252:772-80.

95. Wildi LM, Raynauld JP, Martel-Pelletier J, Abram F, Dorais M, Pelletier JP. Relationship between bone marrow lesions, cartilage loss and pain in knee osteoarthritis: results from a randomised controlled clinical trial using MRI. Ann Rheum Dis. 2010;69:2118-24.

96. Felson DT, Chaisson CE, Hill CL, Totterman SM, Gale ME, Skinner KM, et al. The association of bone marrow lesions with pain in knee osteoarthritis. Ann Intern Med. 2001;134:541-9.

97. Fernandez-Madrid F, Karvonen RL, Teitge RA, Miller PR, Negendank WG. MR features of osteoarthritis of the knee. Magn Reson Imaging. 1994;12:703-9.

98. Gudbergsen H, Lohmander LS, Jones G, Christensen R, Bartels EM, Danneskiold-Samsoe B, et al. Correlations between radiographic assessments and MRI features of knee osteoarthritis - a cross-sectional study. Osteoarthritis Cartilage. 2013;21:535-43.

99. Ip S, Sayre EC, Guermazi A, Nicolaou S, Wong H, Thorne A, et al. Frequency of bone marrow lesions and association with pain severity: results from a population-based symptomatic knee cohort. J Rheumatol. 2011;38:1079-85.

100. Kornaat PR, Watt I, Riyazi N, Kloppenburg M, Bloem JL. The relationship between the MRI features of mild osteoarthritis in the patellofemoral and tibiofemoral compartments of the knee. Eur Radiol. 2005;15:1538-43.

101. Link TM, Steinbach LS, Ghosh S, Ries M, Lu Y, Lane N, et al. Osteoarthritis: MR imaging findings in different stages of disease and correlation with clinical findings. Radiology. 2003;226:373-81.

102. Lo GH, Hunter DJ, Zhang Y, McLennan CE, Lavalley MP, Kiel DP, et al. Bone marrow lesions in the knee are associated with increased local bone density. Arthritis Rheum. 2005;52:2814-21.

103. Torres L, Dunlop DD, Peterfy C, Guermazi A, Prasad P, Hayes KW, et al. The relationship between specific tissue lesions and pain severity in persons with knee osteoarthritis. Osteoarthritis Cartilage. 2006;14:1033-40.

104. Ratzlaff C, Rusell RL, Kwoh K, Hannon M, Grago J, Guermazi A, et al. Quantitative MRI measures of bone marrow lesion volume predict total knee replacement. Osteoarthritis Cartilage. 2014;22:S238-9.

105. Zhao J, Li X, Bolbos RI, Link TM, Majumdar S. Longitudinal assessment of bone marrow edema-like lesions and cartilage degeneration in osteoarthritis using 3 T MR T1rho quantification. Skeletal Radiol. 2010;39:523-31.

106. de Lange BJ, loan-Facsinay A, Bijsterbosch J, Van G, Zuurmond A, Kornaat P, et al. The patellofemoral and femorotibial joints are related based on patterns of MRI features and their association with radiologic progression. Osteoarthritis Cartilage. 2014;22:S254-5.

107. Frobell RB, Nevitt MC, Hudelmaier M, Wirth W, Wyman BT, Benichou O, et al. Femorotibial subchondral bone area and regional cartilage thickness: a cross-sectional description in healthy reference cases and various radiographic stages of osteoarthritis in 1,003 knees from the Osteoarthritis Initiative. Arthritis Care Res. 2010;62:1612-23.

108. Roemer FW, Guermazi A, Niu J, Zhang Y, Mohr A, Felson DT. Prevalence of magnetic resonance imaging-defined atrophic and hypertrophic phenotypes of knee osteoarthritis in a population-based cohort. Arthritis Rheum. 2012;64:429-37.

109. Neogi T, Felson D, Niu J, Lynch J, Nevitt M, Guermazi A, et al. Cartilage loss occurs in the same subregions as subchondral bone attrition: a within-knee subregion-matched approach from the Multicenter Osteoarthritis Study. Arthritis Rheum. 2009;61:1539-44.

110. Reichenbach S, Guermazi A, Niu J, Neogi T, Hunter DJ, Roemer FW, et al. Prevalence of bone attrition on knee radiographs and MRI in a community-based cohort. Osteoarthritis Cartilage. 2008;16:1005-10.

111. Cicuttini FM, Jones G, Forbes A, Wluka AE. Rate of cartilage loss at two years predicts subsequent total knee arthroplasty: a prospective study. Ann Rheum Dis. 2004;63:1124-7.

112. Davies-Tuck ML, Wluka AE, Wang Y, Teichtahl AJ, Jones G, Ding C, et al. The natural history of cartilage defects in people with knee osteoarthritis. Osteoarthritis Cartilage. 2008;16:337-42.

113. Carnes J, Stannus O, Cicuttini F, Ding C, Jones G. Knee cartilage defects in a sample of older adults: natural history, clinical significance and factors influencing change over 2.9 years. Osteoarthritis Cartilage. 2012;20:1541-7.

114. Everhart JS, Siston RA, Flanigan DC. Tibiofemoral subchondral surface ratio (SSR) is a predictor of osteoarthritis symptoms and radiographic progression: data from the Osteoarthritis Initiative (OAI). Osteoarthritis Cartilage. 2014;22:771-8.
115. Kalichman L, Zhang Y, Niu J, Goggins J, Gale D, Felson DT, et al. The association between patellar alignment and patellofemoral joint osteoarthritis features - an MRI study. Rheumatology. 2007;46:1303-8.

116. Stefanik JJ, Roemer FW, Zumwalt AC, Zhu Y, Gross KD, Lynch JA, et al. Association between measures of trochlear morphology and structural features of patellofemoral joint osteoarthritis on MRI: the MOST study. J Orthopaedic Res. 2012;30:1-8.

117. Eckstein F, Wirth W, Hunter DJ, Guermazi A, Kwoh CK, Nelson DR, et al. Magnitude and regional distribution of cartilage loss associated with grades of joint space narrowing in radiographic osteoarthritis - data from the Osteoarthritis Initiative (OAI). Osteoarthritis Cartilage. 2010;18:760-8.

118. Bowes MA, Vincent GR, Wolstenholme CB, Conaghan PG. A novel method for bone area measurement provides new insights into osteoarthritis and its progression. Ann Rheum Dis. 2015;74:519-25.

119. Hunter DJ, Bowes M, Boudreau RM, Hannon MJ, Kwohx KC. Does bone shape predict the development of incident knee oa? Osteoarthritis Cartilage. 2013;21

120. Neogi T, Bowes MA, Niu JB, De Souza KM, Vincent GR, Goggins J, et al. Magnetic resonance imaging-based three-dimensional bone shape of the knee predicts onset of knee osteoarthritis: data from the osteoarthritis initiative. Arthritis Rheum. 2013;65:2048-58.

121. Wluka AE, Wang Y, Davis SR, Cicuttini FM. Tibial plateau size is related to grade of joint space narrowing and osteophytes in healthy women and in women with osteoarthritis. Ann Rheum Dis. 2005;64:1033-7.

122. Crema MD, Roemer FW, Marra MD, Niu J, Lynch JA, Felson DT, et al. Contrast-enhanced MRI of subchondral cysts in patients with or at risk for knee osteoarthritis: the MOST study. Eur J Radiol. 2010;75:e92-6.

123. Lindsey CT, Narasimhan A, Adolfo JM, Jin H, Steinbach LS, Link T, et al. Magnetic resonance evaluation of the interrelationship between articular cartilage and trabecular bone of the osteoarthritic knee. Osteoarthritis Cartilage. 2004;12:86-96.

124. Dore D, Quinn S, Ding C, Winzenberg T, Zhai G, Cicuttini F, et al. Natural history and clinical significance of MRI-detected bone marrow lesions at the knee: a prospective study in community dwelling older adults. Arthritis Res Ther. 2010;12:R223.

125. Moisio K, Eckstein F, Chmiel JS, Guermazi A, Prasad P, Almagor O, et al. Denuded subchondral bone and knee pain in persons with knee osteoarthritis. Arthritis Rheum. 2009;60:3703-10.

126. Zhang Y, Nevitt M, Niu J, Lewis C, Torner J, Guermazi A, et al. Fluctuation of knee pain and changes in bone marrow lesions, effusions, and synovitis on magnetic resonance imaging. Arthritis Rheum. 2011;63:691-9.

127. Ai F, Yu C, Zhang W, Morelli JN, Kacher D, Li X. MR imaging of knee osteoarthritis and correlation of findings with reported patient pain. J Huazhong Univ Sci Technolog Med Sci. 2010;30:248-54.

128. Bilgici A, Dogan C, Cil E, Sakarya S, Kuru O, Selcuk MB. Relationship between pain severity and magnetic resonance imaging features in patients with osteoarthritis of the Knee [Turkish]. Turkish J Rheumatol. 2010;25:184-90.

129. Kim IJ, Kim DH, Jung JY, Song YW, Guermazi A, Crema MD, et al. Association between bone marrow lesions detected by magnetic resonance imaging and knee pain in community residents in Korea. Osteoarthritis Cartilage. 2013;21:1207-13.

130. Kornaat PR, Bloem JL, Ceulemans RY, Riyazi N, Rosendaal FR, Nelissen RG, et al. Osteoarthritis of the knee: association between clinical features and MR imaging findings. Radiology. 2006;239:811-7.

131. Lo GH, McAlindon TE, Niu J, Zhang Y, Beals C, Dabrowski C, et al. Bone marrow lesions and joint effusion are strongly and independently associated with weight-bearing pain in knee osteoarthritis: data from the osteoarthritis initiative. Osteoarthritis Cartilage. 2009;17:1562-9.

132. Ratzlaff C, Guermazi A, Collins J, Katz JN, Losina E, Vanwyngaarden C, et al. A rapid, novel method of volumetric assessment of MRI-detected subchondral bone marrow lesions in knee osteoarthritis. Osteoarthritis Cartilage. 2013;21:806-14.

133. Sharma L, Chmiel JS, Almagor O, Dunlop D, Guermazi A, Bathon JM, et al. Significance of preradiographic magnetic resonance imaging lesions in persons at increased risk of knee osteoarthritis. Arthritis Rheumatol. 2014;66:1811-9.

134. Stefanik J, Gross K, Felson D, Niu J, Zhang Y, Lewis C, et al. Does medial patellofemoral osteoarthritis matter? the relation of MRI-detected structural damage in the medial and lateral patellofemoral joint to knee pain: the MOST and Framingham osteoarthritis studies. Osteoarthritis Cartilage. 2014;22:S54-5.

135. Zhai G, Blizzard L, Srikanth V, Ding C, Cooley H, Cicuttini F, et al. Correlates of knee pain in older adults: Tasmanian older adult cohort study. Arthritis Rheum. 2006;55:264-71. 
136. Sengupta M, Zhang YQ, Niu JB, Guermazi A, Grigorian M, Gale D, et al. High signal in knee osteophytes is not associated with knee pain. Osteoarthritis Cartilage. 2006;14:413-7.

137. Hayashi D, Xu L, Roemer FW, Hunter DJ, Li L, Katur AM, et al. Detection of osteophytes and subchondral cysts in the knee with use of tomosynthesis. Radiology. 2012;263:206-15.

138. Hernandez-Molina G, Neogi T, Hunter DJ, Niu J, Guermazi A, Reichenbach S, et al. The association of bone attrition with knee pain and other MRI features of osteoarthritis. Ann Rheum Dis. 2008;67:43-7.

139. Javaid MK, Kiran A, Guermazi A, Kwoh CK, Zaim S, Carbone L, et al. Individual magnetic resonance imaging and radiographic features of knee osteoarthritis in subjects with unilateral knee pain: The health, aging, and body composition study. Arthritis Rheum. 2012;64:3246-55.

140. Javaid MK, Lynch JA, Tolstykh I, Guermazi A, Roemer F, Aliabadi P, et al. Pre-radiographic MRI findings are associated with onset of knee symptoms: the MOST study. Osteoarthritis Cartilage. 2010;18:323-8.

141. Haugen IK, Slatkowsky-Christensen B, Boyesen P, Sesseng S, van der Heijde D, Kvien TK. MRI findings predict radiographic progression and development of erosions in hand osteoarthritis. Ann Rheum Dis. 2014

142. Haugen IK, Boyesen P, Slatkowsky-Christensen B, Sesseng S, van der Heijde D, Kiven TK. Comparison of features by MRI and radiographs of the interphalangeal finger joints in patients with hand osteoarthritis. Ann Rheum Dis. 2012;71:345-50.

143. Haugen IK, BÃs yesen P, Slatkowsky-Christensen B, Sesseng S, van der Heijde $D$, Kiven TK. Associations between radiographic and clinical osteoarthritis features and MRI-defined bone marrow lesions in the finger joints. Ann Rheum Dis. 2012;71:299.

144. Haugen IK, Slatkowsky-Christensen B, Sesseng S, Kiven TK. Predictive value of MRI-defined synovitis, bone marrow lesions and central erosions on pain and physical function in hand osteoarthritis. Osteoarthritis Cartilage. 2014;22:S386.

145. Haugen IK, Boyesen P, Slatkowsky-Christensen B, Sesseng S, van der Heijde D, Kvien TK. Associations between MRI-defined synovitis, bone marrow lesions and structural features and measures of pain and physical function in hand osteoarthritis. Ann Rheum Dis. 2012;71:899-904.

146. Agricola R, Reijman M, Bierma-Zeinstra SM, Verhaar JA, Weinans $H$, Waarsing JH. Total hip replacement but not clinical osteoarthritis can be predicted by the shape of the hip: a prospective cohort study (CHECK). Osteoarthritis Cartilage. 2013;21:559-64.

147. Agricola R, Heijboer MP, Bierma-Zeinstra SM, Verhaar JA, Weinans $H$, Waarsing $\mathrm{JH}$. Cam impingement causes osteoarthritis of the hip: a nationwide prospective cohort study (CHECK). Ann Rheum Dis. 2013;72:918-23.

148. Agricola R, Heijboer MP, Roze RH, Reijman M, Bierma-Zeinstra SM, Verhaar $J A$, et al. Pincer deformity does not lead to osteoarthritis of the hip whereas acetabular dysplasia does: acetabular coverage and development of osteoarthritis in a nationwide prospective cohort study (CHECK). Osteoarthritis Cartilage. 2013;21:1514-21

149. Gosvig KK, Jacobsen S, Sonne-Holm S, Palm H, Troelsen A. Prevalence of malformations of the hip joint and their relationship to sex, groin pain, and risk of osteoarthritis: a population-based survey. J Bone Joint Surg Am. 2010;92:1162-9.

150. Barr RJ, Gregory JS, Reid DM, Aspden RM, Yoshida K, Hosie G, et al. Predicting OA progression to total hip replacement: can we do better than risk factors alone using active shape modelling as an imaging biomarker? Rheumatology (Oxford). 2012;51:562-70.

151. Kumar D, Wyatt CR, Lee S, Nardo L, Link TM, Majumdar S, et al. Association of cartilage defects, and other MRI findings with pain and function in individuals with mild-moderate radiographic hip osteoarthritis and controls. Osteoarthritis Cartilage. 2013;21:1685-92.

152. Maksymowych WP, Cibere J, Loeuille D, Weber U, Zubler V, Roemer FW, et al. Preliminary validation of 2 magnetic resonance image scoring systems for osteoarthritis of the hip according to the OMERACT filter. J Rheumatol. 2014:41:370-8.

153. Radin EL, Rose RM. Role of subchondral bone in the initiation and progression of cartilage damage. Clin Orthop Relat Res. 1986;34-40.

154. Suri S, Walsh DA. Osteochondral alterations in osteoarthritis. Bone. 2012;51:204-11.

155. Burr DB. Anatomy and physiology of the mineralized tissues: role in the pathogenesis of osteoarthrosis. Osteoarthritis Cartilage. 2004;12:S20-30.

156. Hayami T, Pickarski M, Zhuo Y, Wesolowski GA, Rodan GA, Duong le T, Characterization of articular cartilage and subchondral bone changes in the rat anterior cruciate ligament transection and meniscectomized models of osteoarthritis. Bone. 2006;38:234-43.
157. Bettica P, Cline G, Hart DJ, Meyer J, Spector TD. Evidence for increased bone resorption in patients with progressive knee osteoarthritis: longitudinal results from the Chingford study. Arthritis Rheum. 2002:46:3178-84.

158. Hunter DJ, Gerstenfeld L, Bishop G, David AD, Mason ZD, Einhorn TA, et al. Bone marrow lesions from osteoarthritis knees are characterized by sclerotic bone that is less well mineralized. Arthritis Res Ther. 2009;11.

159. Day JS, Ding M, van der Linden JC, Hvid I, Sumner DR, Weinans H. A decreased subchondral trabecular bone tissue elastic modulus is associated with pre-arthritic cartilage damage. J Orthop Res. 2001;19:914-8.

160. Roemer F, Felson DT, Wang K, Crema M, Marra MD, Zhang Y, et al. Co-localization of non-cartilaginous articular pathology and cartilage damage in regard to subsequent cartilage loss in subjects with or at risk for knee osteoarthritis - The most study. Ann Rheum Dis. 2013;72:942-8.

161. Pelletier JP, Roubille C, Raynauld JP, Abram F, Dorais M, Delorme P, et al. Disease-modifying effect of strontium ranelate in a subset of patients from the Phase III knee osteoarthritis study SEKOIA using quantitative MRI: reduction in bone marrow lesions protects against cartilage loss. Ann Rheum Dis. 2015;74:422-9.

162. Henriksen M, Christensen R, Hunter DJ, Gudbergsen H, Boesen M, Lohmander LS, et al. Structural changes in the knee during weight loss maintenance after a significant weight loss in obese patients with osteoarthritis: a report of secondary outcome analyses from a randomized controlled trial. Osteoarthritis Cartilage. 2014;22:639-46.

163. Kwoh CK, Roemer FW, Hannon MJ, Moore CE, Jakicic JM, Guermazi A, et al, Effect of oral glucosamine on joint structure in individuals with chronic knee pain: a randomized, placebo-controlled clinical trial. Arthritis Rheumatol. 2014;66:930-9.

164. Miura $H$, Noguchi $Y$, Mitsuyasu $H$, Nagamine $R$, Urabe $K$, Matsuda $S$, et al. Clinical features of multiple epiphyseal dysplasia expressed in the knee. Clin Orthop Relat Res. 2000;184-90.

165. Kalichman L, Zhang Y, Niu J, Goggins J, Gale D, Zhu Y, et al. The association between patellar alignment on magnetic resonance imaging and radiographic manifestations of knee osteoarthritis. Arthritis Res Ther. 2007:9.

166. Bowes M, Lohmander S, Wolstenholme C, Vincent G, Frobell R. Significant change of bone shape occur over the first five years after acl injury. Osteoarthritis Cartilage. 2013;21:S220.

167. Barr ADB, Hensor E, Kingsbury S, Peat G, Sharples L, Bowes M, et al. Three-dimensional magnetic resonance imaging knee bone shape predicts total knee replacement: data from the osteoarthritis initiative. Ann Rheum Dis. 2015;74:185.

168. Roemer FW, Neogi T, Nevitt MC, Felson DT, Zhu Y, Zhang Y, et al. Subchondral bone marrow lesions are highly associated with, and predict subchondral bone attrition longitudinally: the MOST study. Osteoarthritis Cartilage. 2010;18:47-53.

169. Bobinac D, Spanjol J, Zoricic S, Maric I. Changes in articular cartilage and subchondral bone histomorphometry in osteoarthritic knee joints in humans. Bone. 2003;32:284-90.

170. Ding M, Odgaard A, Hvid I. Changes in the three-dimensional microstructure of human tibial cancellous bone in early osteoarthritis. J Bone Joint Surg Br. 2003:85:906-12.

171. Li B, Aspden RM. Composition and mechanical properties of cancellous bone from the femoral head of patients with osteoporosis or osteoarthritis. J Bone Miner Res. 1997;12:641-51.

172. Phan CM, Link TM, Blumenkrantz G, Dunn TC, Ries MD, Steinbach LS, et al. MR imaging findings in the follow-up of patients with different stages of knee osteoarthritis and the correlation with clinical symptoms. European Radiology. 2006;16:608-18.

173. Kornaat PR, Kloppenburg M, Sharma R, Botha-Scheepers SA, Le MPM, Coene $L N$, et al. Bone marrow edema-like lesions change in volume in the majority of patients with osteoarthritis; associations with clinical features. European Radiology. 2007;17:3073-8.

174. Davies-Tuck ML, Wluka AE, Wang Y, English DR, Giles GG, Cicuttini F. The natural history of bone marrow lesions in community-based adults with no clinical knee osteoarthritis. Ann Rheum Dis. 2009;68:904-8.

175. Laslett LL, Dore DA, Quinn SJ, Boon P, Ryan E, Winzenberg TM, et al. Zoledronic acid reduces knee pain and bone marrow lesions over 1 year: a randomised controlled trial. Ann Rheum Dis. 2012;71:1322-8.

176. Hunter DJ, Beavers D, Eckstein F, Guermazi A, Loeser RF, Nicklas BJ, et al. The intensive diet and exercise for arthritis trial (IDEA): 18-month radiographic and MRI outcomes. Arthritis Rheum. 2012;64:S1070. 
177. Arnoldi CC, Linderholm H, Mussbichler H. Venous engorgement and intraosseous hypertension in osteoarthritis of the hip. J Bone Joint Surg Br. 1972;54:409-21.

178. Burr DB. The importance of subchondral bone in the progression of osteoarthritis. J Rheumatol Suppl. 2004;70:77-80.

179. Simkin PA. Bone pain and pressure in osteoarthritic joints. Novartis Found Symp. 2004;260:179-86. discussion 86-90, 277-9.

180. Hudelmaier M, Wirth W, Nevitt M, Eckstein F. Longitudinal rates of change in subchondral bone size in healthy knees and knees with radiographic osteoarthritis. Osteoarthritis Cartilage. 2013;21:S242.

181. Felson DT, Niu J, Guermazi A, Roemer F, Aliabadi P, Clancy M, et al.

Correlation of the development of knee pain with enlarging bone marrow lesions on magnetic resonance imaging. Arthritis Rheum. 2007;56:2986-92.

\section{Submit your next manuscript to BioMed Central} and take full advantage of:

- Convenient online submission

- Thorough peer review

- No space constraints or color figure charges

- Immediate publication on acceptance

- Inclusion in PubMed, CAS, Scopus and Google Scholar

- Research which is freely available for redistribution 